\title{
NANOTRIBOLOGICAL CHARACTERIZATION OF HUMAN HAIR AND SKIN USING ATOMIC FORCE MICROSCOPY (AFM)
}

\author{
A Thesis \\ Presented in Partial Fulfillment of the Requirements for \\ the Degree Master of Science in the \\ Graduate School of The Ohio State University
}

By

Carmen Anthony LaTorre, B.S.

$* * * * *$

The Ohio State University

2005

Master's Examination Committee:

Professor Bharat Bhushan, Adviser

Approved by

Professor Mark Walter

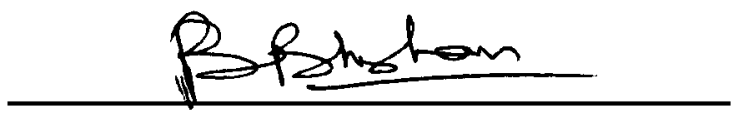

Adviser

Mechanical Engineering Graduate Program 


\section{ABSTRACT}

Human hair is a nanocomposite biological fiber. Maintaining the health, feel, shine, color, softness, and overall aesthetics of the hair is highly desired. Hair care products such as shampoos and conditioners, along with damaging processes such as chemical dyeing and permanent wave treatments, affect the maintenance and grooming process and are important to study because they alter many hair properties. Nanoscale characterization of the morphological, frictional, and adhesive properties (tribological properties) of hair are essential to evaluate and develop better cosmetic products, and to advance the understanding of biological and cosmetic science. The atomic/friction force microscope (AFM/FFM) has recently become an important tool for studying the micro/nanoscale properties of human hair. This thesis presents a comprehensive review of tribological properties of various hair and skin as a function of ethnicity, damage, conditioning treatment, and various environments. Nanotribological properties such as roughness, friction, adhesion, and wear are presented, as well as investigations of scale effects and directionality dependence on friction and adhesion. 
Dedicated to my parents, with love and gratitude 


\section{ACKNOWLEDGMENTS}

I wish to thank my adviser, Professor Bharat Bhushan, for allowing me this opportunity to perform research in his renowned laboratory. It has been a wonderful experience, both personally and professionally. Above all else, I am grateful to him for teaching me the importance of performing engineering research with accountability, creativity, enthusiasm, and a systematic process. It is truly my hope that someday I will excel in these areas as well as he has.

A special thanks is given to Professor Mark Walter for serving on my examination committee. As my freshman year statics professor, his advice helped me choose mechanical engineering, and as my undergraduate research adviser, his guidance helped me choose to go on to (and be prepared for!) graduate school.

I thank my wonderful colleagues in the NLIM laboratory, past and present, for their friendship, good spirits, and technical guidance: Dr. Huiwen Liu, Dr. Toshi Kasai, Dr. Nikhil Tambe, Dr. Guohua Wei, Dr. Zhenhua Tao, Dr. Michael Reinstaedtler, Walt Hanson, and Zach Burton, among many others. I have enjoyed all the day-to-day interactions, discussions, and fun times I have had with these exceptional people.

I am very grateful to Dr. Peter Torgerson and Dr. Jian Yang of Procter \& Gamble for their continuous support and encouragement throughout the project. Our many technical discussions were essential to the understanding and completion of this research. 
I also thank Dr. Rob Willicut and Dr. Matt Wagner of P\&G for their many helpful comments.

Most of all, I would like to thank my parents, Jack and Denise LaTorre, for teaching me the importance of following my dreams, working hard, and being a person of character. I love you both very much.

Finally, I thank Jen for her unwavering support, love, and faith in me. You never cease to amaze me, and I could not have done this without you. 


\section{VITA}

May 9, $1981 \ldots \ldots \ldots \ldots \ldots \ldots \ldots \ldots \ldots \ldots \ldots$ Born - Warren, Michigan, USA

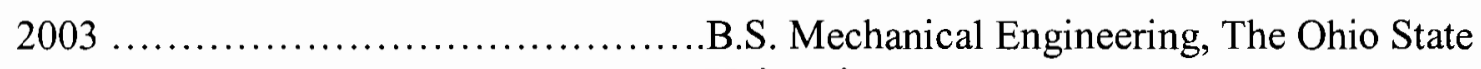
University.

2003 - present..........................Graduate Research Associate, The Ohio State University

\section{FIELDS OF STUDY}

Major Field: Mechanical Engineering 


\section{TABLE OF CONTENTS}

Page

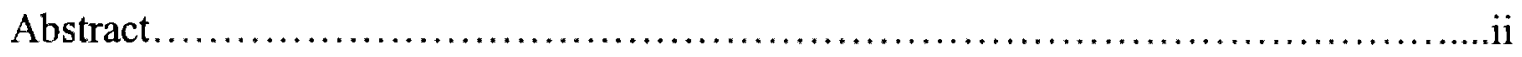

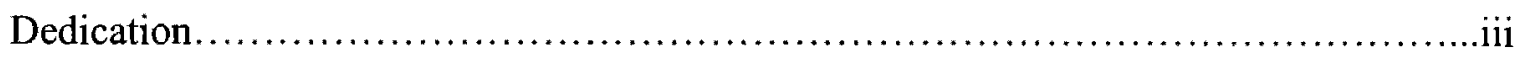

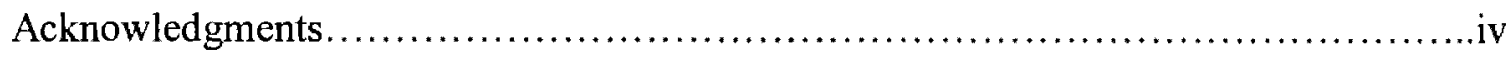

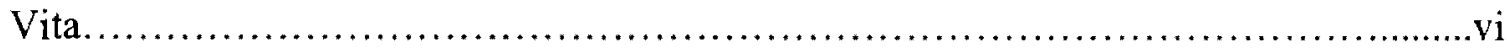

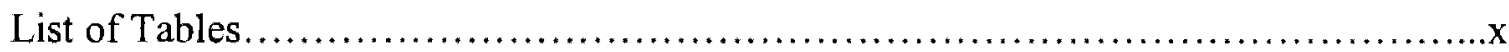

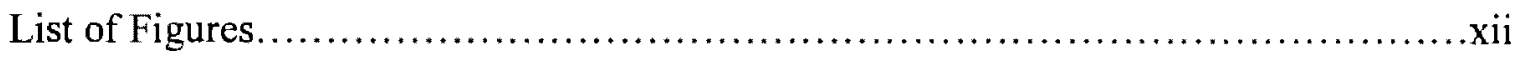

Chapters:

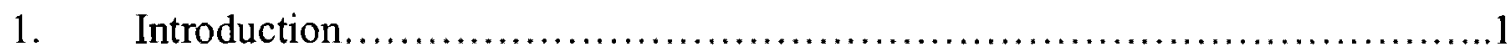

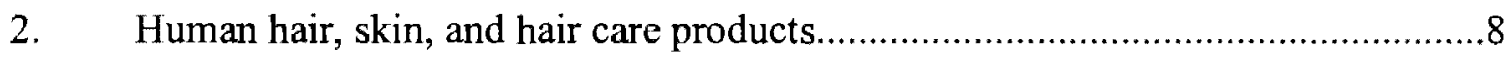

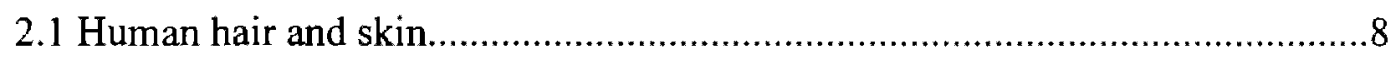

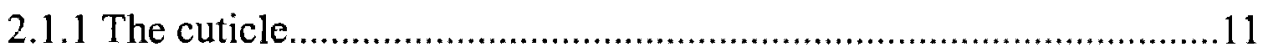

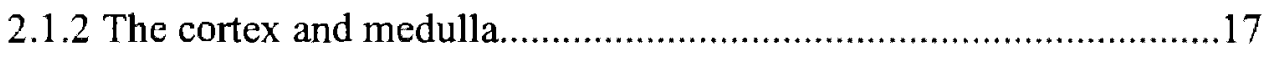

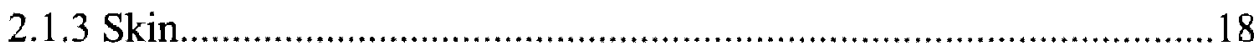

2.2 Hair care: Cleaning and conditioning treatments, and damaging processes....21

2.2.1 Cleaning and conditioning treatments: shampoo and conditioner....21

2.2.1.1 Shampoo: constitution and main functions. .22 


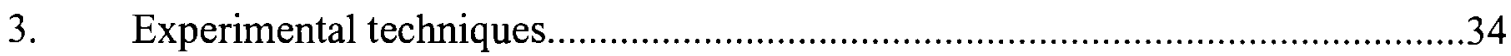

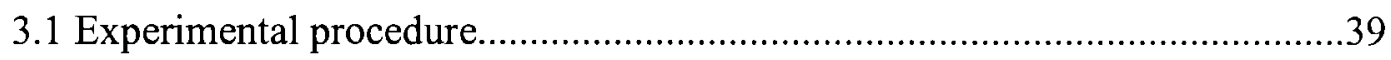

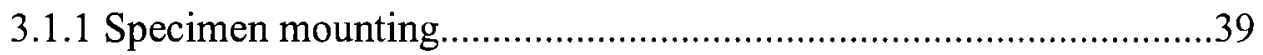

3.1.2 Nanotribological property measurements........................................39

3.1.3 Relative humidity, temperature, and soaking, and durability measurements.

3.1.4 Microscale and macroscale friction and adhesion measurements for scale effect study

4. Results and discussion

4.1 Surface roughness, friction, and adhesion for various ethnicities of hair........51

4.1.1 Directionality effects of friction on the nanoscale

4.2 Surface roughness, friction, and adhesion for virgin and chemically damaged Caucasian hair (with and without commercial conditioner treatment). 69

4.2.1 Effect of relative humidity, temperature, and soaking, and durability measurements. 
4.3 Surface roughness, friction, and adhesion for hair treated with various combinations of conditioner ingredients.

4.4 Investigation of directionality dependence and scale effects on friction and adhesion of hair

4.4.1 Directionality dependence.

4.4.2 Scale effects 102

4.5 Surface roughness and friction of skin.

5. Conclusions

List of references. 126

Appendix A. Shampoo and conditioner treatment procedure. 130 Appendix B. Conditioner thickness approximation 132 


\section{LIST OF TABLES}

Table Page

1.1 Common hair care products/processes and their functions................................

1.2 Desired features and corresponding tribological attributes of conditioners..........4

2.1 Summary of chemical species presented in human hair....................................10

2.2 Various layers of the cuticle and their details...................................................11

2.3 Variation in cross-sectional dimensions of human hair.....................................17

2.4 Components of common shampoos and their functions..................................24

2.5 Combinations of conditioner ingredients and their benefits towards wet and dry

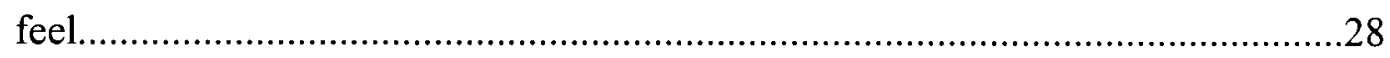

2.6 Chemical structure and purpose/function of conditioner ingredients...................30

3.1 Comparison of methods used to characterize hair on micro/nanoscale.................35

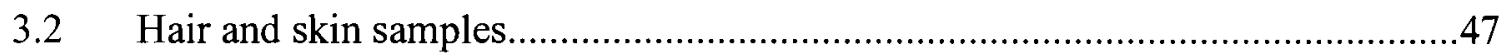

3.3 Matrix of hair samples treated with various combinations of ingredients............49

3.4 Contact angle and surface energy of relevant materials associated with

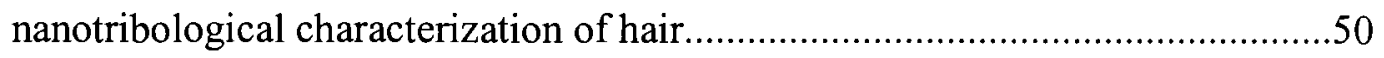

4.1 Surface roughness, coefficient of friction, and adhesive force values of virgin, chemo-mechanically damaged, and virgin treated (1 cycle commercial conditioner) hair at each ethnicity......

4.2 Nanotribological parameters for virgin, chemically damaged, and treated hair...76 
4.3 Observations and corresponding mechanisms regarding coefficient of friction and adhesion for various hair treatments. .92

4.4 Coefficient of friction and adhesive force values showing directionality dependence on the microscale. This dependence has also been observed on the nano- and macroscale. 102

4.5 Coefficient of friction and adhesive force of hair on various scales 110

4.6 Observations and corresponding mechanisms regarding coefficient of friction and adhesion for various hair treatments. 116

4.7 Surface roughness parameters $\sigma, \beta^{*}$ for collagen and polyurethane films and human skin. 


\section{LIST OF FIGURES}

Figure

Page

1.1 Schematics illustrating various functions with associated macroscale and micro/nanoscale mechanisms of hair and skin friction during feel or touch, shaking and bouncing of the hair, combing, and entanglement [LaTorre and Bhushan, 2005a]

2.1 Schematic of hair fiber structure and cuticle sublamellar structure. ....................9

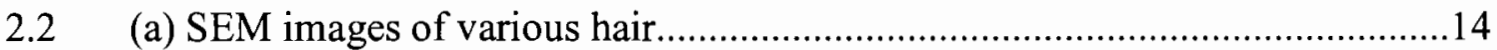

(b) SEM images of virgin Caucasian hair at three locations...............................15

(c) SEM images of Caucasian, virgin and treated hair [Wei et al., 2005]........16

2.3 AFM images of various virgin hair [LaTorre and Bhushan, 2005a]..................19

2.4 (a) SEM images of virgin hair cross-section (in the figure EXO, END, and CMC stand for exocuticle, endocuticle, and cell membrane complex, respectively) [Wei

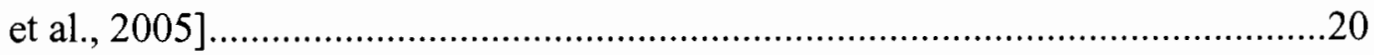

(b) TEM of hair cross-section.

2.5 Schematic image of human skin structure with different layers: dermis, epidermis, and horny layer (L'Oreal).

2.6 Negatively charged hair and the deposition of positively charged conditioner on the cuticle surface [LaTorre et al., 2006]

2.7 Conditioner formation from emulsion to gel network .28 
3.1 Schematic diagram of AFM operation with human hair sample.

3.2 Comparison of macroscale and micro/nanoscale friction test apparatuses............38

3.3 Interactions between the AFM tip and conditioner on the cuticle surface for treated hair [LaTorre and Bhushan, 2005b]....

3.4 Typical force calibration plot for Caucasian virgin hair. Contact between the tip and hair occurs at point $\mathrm{B}$. At point $\mathrm{C}$, the elastic force of the cantilever becomes equivalent to the adhesive force, causing the cantilever to snap back to D.

4.1 Surface roughness of virgin and chemo-mechanically damaged Caucasian, Asian, and African hair at various scan sizes [LaTorre and Bhushan, 2005a]. .52

4.2 (a) Surface roughness and friction images for virgin and chemo-mechanically damaged Caucasian, Asian hair at 5 and $10 \mu \mathrm{m}^{2}$ scan sizes. Shown above each image is a cross section taken at the corresponding arrows to show roughness and friction force information.

(b) Surface roughness and friction force images for virgin and chemomechanically damaged African hair at 5 and $10 \mu \mathrm{m}^{2}$ scan sizes. Shown above each image is a cross section taken at the corresponding arrows to show roughness and friction force information [LaTorre and Bhushan, 2005a]. .55

4.3 (a) Surface roughness and friction images for virgin and virgin treated Caucasian, Asian hair at 5 and $10 \mu \mathrm{m}^{2}$ scan sizes. Shown above each image is a cross section taken at the corresponding arrows to show roughness and friction force 
information.

(b) Surface roughness and friction force images for virgin and virgin treated African hair at 5 and $10 \mu \mathrm{m}^{2}$ scan sizes. Shown above each image is a cross section taken at the corresponding arrows to show roughness and friction force information [LaTorre and Bhushan, 2005a]

4.4 Friction force vs. normal load curves showing typical values for virgin, chemomechanically damaged, and virgin treated Caucasian hair. Error bars represent \pm 1 $\sigma$ on the average coefficient value. In the bottom plot, average coefficient of friction values for virgin, damaged, and treated hair at each ethnicity [LaTorre and Bhushan, 2005a] 62

4.5 Surface roughness, coefficient of friction, and adhesive force data for virgin, chemo-mechanically damaged, and virgin treated hair at each ethnicity [LaTorre and Bhushan, 2005a]

4.6 Force volume maps of virgin, chemo-mechanically damaged, and virgin treated hair at each ethnicity. Examples of the individual force calibration plots, which make up the FV maps, are presented for Caucasian hair of each type [LaTorre and Bhushan, 2005a] 66

4.7 Surface roughness, friction force, and slope across a cuticle scale edge to show the directionality dependence of friction. Left: Surface roughness and friction force mappings with accompanying 2D profiles. Right: Surface slope mappings 
with accompanying 2D profiles [LaTorre and Bhushan, 2005a]

4.8 The effect of damage to the cuticle scales and the deposition of conditioner on the cuticle surface. The cross-section of the hair with and without conditioner is shown below [LaTorre et al., 2006].

4.9 Surface roughness and friction force images for virgin, virgin treated, damaged, damaged treated ( 1 cycle conditioner), and damaged treated ( 3 cycles conditioner) hair at $5 \mu \mathrm{m}$ scan sizes [LaTorre and Bhushan, 2005b]...

4.10 Adhesive force maps displaying variations in adhesive force on the cuticle surface of various hair [LaTorre and Bhushan, 2005b]. .75

4.11 Surface roughness, coefficient of friction, and adhesive force plots for various hair [LaTorre and Bhushan, 2005b].

4.12 Effect of relative humidity on nanotribological properties of various hair [LaTorre and Bhushan, 2005b]. .80

4.13 Effect of temperature on nanotribological properties of various hair [LaTorre and Bhushan, 2005b]. .81

4.14 Effect of soaking in de-ionized water on coefficient of friction and adhesive force for virgin, chemically damaged, and chemically damaged treated (1 cycle commercial) hair [LaTorre et al., 2006]. .82

4.15 Durability study of friction force change as a function of AFM tip cycling for various hair. The images above the plot signify before and after comparisons of a 
cuticle surface subjected to cycling at a $10 \mu \mathrm{N}$ load [LaTorre and Bhushan, $2005 b]$ 83

4.16 Surface roughness and friction force maps of Caucasian chemically damaged hair with various treatments [LaTorre et al., 2006]................................................86

4.17 (a) Adhesive force maps for damaged hair with various treatments....................88

(b) Adhesive force histograms for various hair [LaTorre et al., 2006]................89

4.18 Coefficient of friction, adhesive force, and surface roughness plots for damaged hair with various treatments [LaTorre et al., 2006]..........................................91

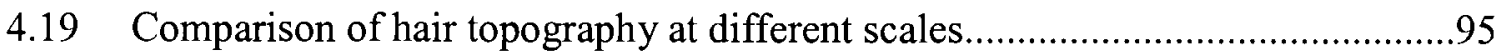

4.20 (a) Directionality effects of hair friction on various scales...............................100

(b) Microscale coefficient of friction data for various hair showing directionality effects. 101

4.21 (a) Friction force vs. normal force curves for micro- and nanoscale coefficient of friction 106

(b) Adhesive force comparison of virgin and virgin treated hair using micro- and nanoscale AFM tips and force calibration plot technique.

4.22 Summary of coefficient of friction and adhesive force data on various scales...109

4.23 Schematic of various mechanisms responsible for scale dependence of hair friction and adhesion.

4.24 (a) Surface roughness and friction force images for collagen and polyurethane 
films at 5 and $10 \mu \mathrm{m}^{2}$ scan sizes. Shown above each image is a cross section taken at the corresponding arrows to show roughness and friction force information

(b) Surface roughness for human skin at 5 and $10 \mu \mathrm{m}^{2}$ scan sizes [LaTorre and Bhushan, 2005a]. 118

4.25 Average coefficient of friction values for collagen and polyurethane films. Error bars represent $\pm 1 \sigma$ on the average coefficient value [LaTorre and Bhushan, 2005a] 


\section{CHAPTER 1}

\section{INTRODUCTION}

Everybody wants beautiful, healthy hair and skin. For most people, grooming and maintenance of hair and skin is a daily process. The demand for products that improve the look and feel of these surfaces has created a huge industry for hair and skin care. Beauty care technology has advanced the cleaning, protection, and restoration of desirable hair and skin properties by altering the hair surface. For many years, especially in the second half of the twentieth century, scientists have focused on the physical and chemical properties of hair to consistently develop products which alter the health, feel, shine, color, softness, and overall aesthetics of the hair. Table 1.1 displays common products and processes involved in hair care. Hair care products such as shampoos and conditioners aid the maintenance and grooming process. Mechanical processes such as combing, cutting, and blowdrying serve to style the hair. Chemical products and processes such as chemical dyes, colorants, bleaches, and permanent wave treatments enhance the appearance and hue of the hair. Of particular interest is how all these common hair care items deposit onto and change hair properties, since these properties are closely tied to product performance. The fact that companies like Procter \& Gamble, L'Oreal, and Unilever have hair care product sales consistently measured in the billions of dollars (http://www.pg.com; http://www.loreal.com; http://unilever.com) suggests that 
understanding the science behind human hair has more than just purely academic benefits, as well.

\begin{tabular}{|l|l|}
\hline Product/Process & Functions \\
\hline Shampoos & Clean the hair and skin of oils \\
\hline Conditioners & $\begin{array}{l}\text { Repair hair damage and make the hair easier to } \\
\text { comb; prevent flyaway; add feel, shine, softness }\end{array}$ \\
\hline Combing/ cutting/ blowdrying & Style the hair \\
\hline Chemical dyes/ colorants/ bleaches & Enhance or change the color and look of hair \\
\hline Permanent wave treatments & Change the style and look of the hair \\
\hline
\end{tabular}

Table 1.1 Common hair care products/processes and their functions

The tribology of the hair changes as a function of the various hair care products and processes. Figure 1.1 illustrates schematically various functions, along with the macro- and micro/nanoscale mechanisms behind these interactions, that make surface roughness, friction, and adhesion very important to hair and skin [LaTorre and Bhushan, 2005a]. Desired features and corresponding tribological attributes of conditioners are listed in Table 1.2 [LaTorre et al., 2006]. Friction is the most relevant parameter to hair care. Our perception of a soft, silky feel comes from our ability to glide a comb or skin over the hair fibers. For a smooth wet and dry feel, friction between hair and skin should be minimized in wet and dry environments, respectively. For a good feel with respect to bouncing and shaking of the hair during walking or running, friction between hair fibers and groups of hair fibers should be low. The friction one feels during combing is a result 
Various functions requiring low friction and adhesion
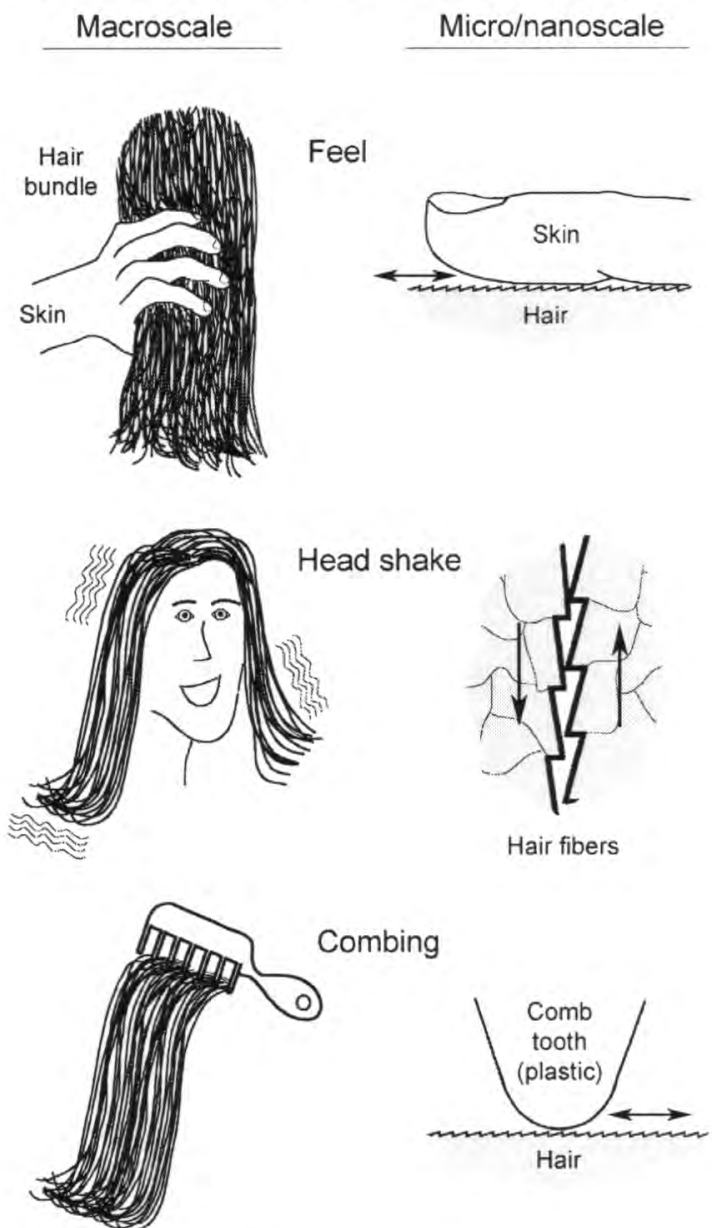

Entanglement

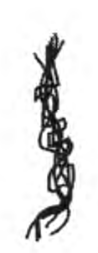

Tangled hair

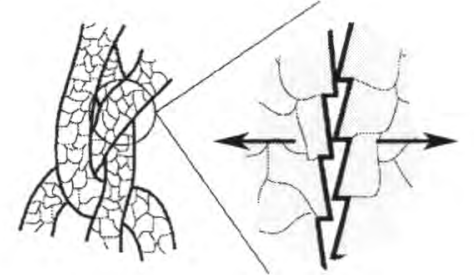

Hair fibers

Fig. 1.1 Schematics illustrating various functions with associated macroscale and micro/nanoscale mechanisms of hair and skin friction during feel or touch, shaking and bouncing of the hair, combing, and entanglement [LaTorre and Bhushan, 2005a]. 


\begin{tabular}{|c|c|}
\hline Desired hair feature & Tribological attributes \\
\hline Smooth feel in wet and dry environments & $\begin{array}{c}\text { Low friction between hair and skin in } \\
\text { respective environment }\end{array}$ \\
\hline Shaking and bouncing during daily \\
activities & $\begin{array}{c}\text { Low friction between hair fibers and } \\
\text { groups of hair }\end{array}$ \\
\hline Easy combing and styling & $\begin{array}{c}\text { Low friction between hair and comb } \\
\text { (plastic) and low adhesion. Note: more } \\
\text { complex styles may require higher } \\
\text { adhesion between fibers. }\end{array}$ \\
\hline
\end{tabular}

Table 1.2 Desired features and corresponding tribological attributes of conditioners

of interactions between hair and the comb material (generally a plastic), and this too needs to be low to easily maintain, sculpt, and comb the hair. Adhesion is also important. To minimize entanglement, adhesive force (the force required to separate the hair fibers) needs to be low. In other cases, a certain level of adhesion may be acceptable and is often a function of the hair style. For individuals seeking "hair alignment", where hair fibers lay flat and parallel to each other, a small amount of adhesive force between fibers may be desired. For more complex and curly styles, even higher adhesion between fibers may be optimal.

Early research into human hair was done primarily on the chemical and physical properties of the hair fiber itself. Key topics dealt with analysis of chemical composition in the fiber, microstructure, and hair growth, to name a few. Mechanical properties were also of interest. Most of the mechanical property studies of human hair were on the macroscale and used conventional methods, such as tension, torsion and bending tests [Robbins, 1994; Feughelman, 1997; Swift, 1999, 2000; Barnes and Roberts, 2000; 
Jachowitz and McMullen, 2002]. Efforts were also made to study the effects of environmental and chemical damage and treatment, such as dyeing, bleaching, and polymer application; these topics have stayed a mainstream area of investigation due to the availability and formation of new chemicals and conditioning ingredients. Tribology has generally been studied via macroscale friction force of hair. As a matter of fact, much of the tribological work performed by the hair care industry today still focuses on the measurement of macroscale friction, particularly between a skin replica and a hair switch of interest [Robbins, 1994]. The intrinsic differences of the hair as a function of ethnicity eventually became a concern as well. For instance, research has shown that African-American hair has higher resistance to combing, higher static charge, and lower moisture content than Caucasian hair [Syed et al., 1996]. Because of differences like these, a growing number of hair care products specifically tailored for ethnic hair care have been developed and sold with commercial success.

Modern research since late 1990 s has been primarily concerned with using micro/nanoscale experimental methods such as atomic force/ friction force microscopies (AFM/ FFM) to answer the complex questions surrounding the structure and behavior of the hair. Nanoscale characterization of the cellular structure, mechanical properties, and tribological properties of hair are essential to evaluate and develop better cosmetic products, and to advance the understanding of composite biological systems, cosmetic science, and dermatology.

AFM/FFM have been used to effectively study the structure of the hair surface and cross-section. AFM provides the potential for being able to see the cellular structure and molecular assembly of hair, for determining various properties of hair, such as elastic 
modulus and viscoelastic properties, and for investigating the physical behavior of various cellular structure of hair in various environments [Chen and Bhushan, 2005a, b]. As a non-invasive technique, AFM has been used to evaluate the effect of hair treatment and can be operated in ambient conditions in order to study the effect of environment on various physical properties. AFM has also been used to study nanotribological properties of hair and skin and the effects of hair care products on hair [LaTorre and Bhushan, 2005a, b; LaTorre et al., 2006]. Roughness parameters have been measured to compare changes due to damaging processes. Nanoscale friction force has been measured to understand damage or conditioner distribution and its effect on hair tribology. Adhesive force mapping has shown useful to observe the conditioner distribution as well.

It is known that adhesion, friction, and lubrication of many interfaces are scale dependant [Bhushan, 1999b, 2005; Bhushan et al., 2004]. In the case of hair, the magnitude of nanoscale coefficient of friction in LaTorre and Bhushan [2005a] was lower than the macroscale values found in Bhushan et al. [2005] for the same hair sets. It was also found that there are both similarities and differences when comparing the trends for various hair at both scales. Also, directionality effects of friction were observed on both scales, but via slightly different mechanisms due to the size of the contacts involved [LaTorre and Bhushan, 2006]. It is thus recognized that scale effects are an important aspect of studying the tribology of hair. However, no microscale tribological data for hair exists in literature. This is unfortunate because many interactions between hair-skin, hair-comb, and hair-hair contact takes place at microasperities ranging from a few $\mu \mathrm{m}$ to hundreds of $\mu \mathrm{m}$. For instance, the skin on ones finger is not microscopically smooth but is instead patterned with bumps and ridges that contact the hair surface. Also, adhesion 
may be very relevant on the microscale because often many small patches of cuticle scales (5-10 $\mu \mathrm{m}$ long) from two different fibers may be in contact at the same time. Thus, further the scale effect study and to bridge the gap between the macro- and nanoscale data, as well as to gain a full understanding of the mechanisms behind the trends, it is worthwhile to look at hair tribology on the microscale as well.

This thesis presents a comprehensive study of various hair and skin nanotribological properties as a function of ethnicity, damage, conditioning treatment, and various environments. Nanotribological properties including surface roughness, friction, adhesion, and wear are presented, as well as investigations of scale effects and directionality dependence on friction and adhesion. 


\section{CHAPTER 2}

\section{HUMAN HAIR, SKIN, AND HAIR CARE PRODUCTS}

\subsection{Human hair and skin}

Figure 2.1 shows a schematic of a human hair fiber with its various layers of cellular structure [Zviak, 1986; Robbins, 1994; Feughelman, 1997; Jolles et al., 1997]. Hair fibers (about 50 to $100 \mu \mathrm{m}$ in diameter) consist of the cuticle and cortex, and in some cases medulla in the central region. All are composed of dead cells, which are mainly filled with keratin protein. Table 2.1 displays a summary of the chemical species of hair [Chen and Bhushan, 2005a]. Depending on its moisture content, human hair consists of approximately $65-95 \%$ proteins, which are condensation polymers of amino acids. The remaining constituents are water, lipids (structural and free), pigment, and trace elements. Among numerous amino acids in human hair, cystine is one of the most important amino acids. The distinct cystine content of various cellular structure of human hair results in a significant effect on their physical properties. Cystine has the capacity to crosslink the protein by its intermolecular disulfide linkages. A high cystine content corresponds to rich disulfide cross-links, leading to high mechanical properties. In addition to disulfide bonds, hair is also rich in peptide bonds and the abundant CO-and $\mathrm{NH}$ - groups present give rise to hydrogen bonds between groups of neighboring chain 
Schematic of hair fiber structure

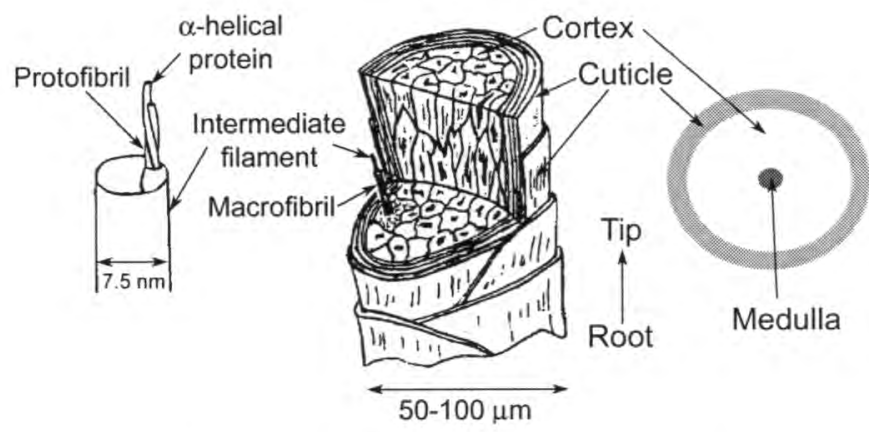

Schematic of the sublamellar structure of the human hair cuticle

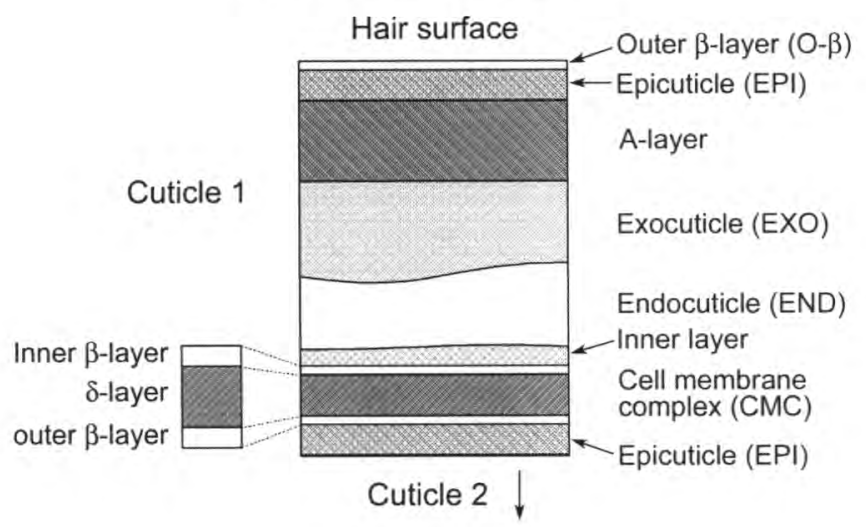

Fig. 2.1 Schematic of hair fiber structure and cuticle sublamellar structure. 


\begin{tabular}{|c|c|}
\hline Keratin protein & $65-95 \%$ \\
\hline (Amino acids) & ${ }^{\oplus} \mathrm{NH}_{3}-\underset{\mathrm{CO}_{2}^{\ominus}}{\mathrm{CH}-\mathrm{R}}$ (R: functional group) \\
\hline Cystine & 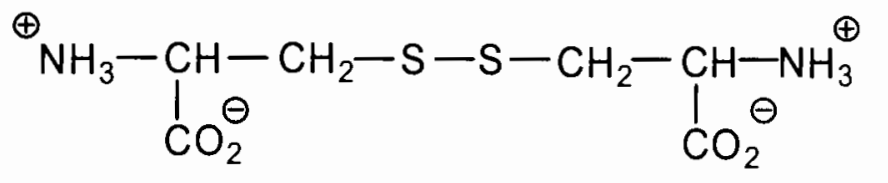 \\
\hline Lipids & Structural and free \\
\hline $\begin{array}{l}\text { 18-methyl eicosanoic } \\
\text { acid (18-MEA) }\end{array}$ & $\mathrm{H}_{3} \mathrm{C} \widetilde{\mathrm{CH}_{3}}$ \\
\hline Water & Up to $30 \%$ \\
\hline $\begin{array}{l}\text { Pigment and trace } \\
\text { elements }\end{array}$ & Melanin \\
\hline
\end{tabular}

Table 2.1 Summary of chemical species presented in human hair.

molecules. The species responsible for color in hair is the pigment - melanin, which is located in the cortex of the hair in granular form.

An average head contains over 100,000 hair follicles, which are the cavities in the skin surface from which hair fibers grow. Each follicle grows about 20 new hair fibers in a lifetime. Each fiber grows for several years until it falls out and is replaced by a new fiber. Hair typically grows at a rate on the order of $10 \mathrm{~mm} / \mathrm{month}$. 


\subsubsection{The cuticle}

The cuticle consists of flat overlapping cells (scales). The cuticle cells are attached at the root end and they point forward the tip end of the hair fiber, like shingles on a roof. Each cuticle cell is approximately 0.3 to $0.5 \mu \mathrm{m}$ thick and the visible length of each cuticle cell is approximately 5 to $10 \mu \mathrm{m}$. The cuticle in human hair is generally 5 to 10 scales thick. Each cuticle cell consists of various sublamellar layers (the epicuticle, the A-layer, the exocuticle, the endocuticle and inner layer) and the cell membrane complex (see Figure 2.1). Table 2.2 displays the various layers of the cuticle, their respective cystine levels [Robbins, 1994], and other details.

\begin{tabular}{|l|c|l|}
\hline \multicolumn{1}{|c|}{ Cuticle layer } & Cystine component & \multicolumn{1}{c|}{ Details } \\
\hline Epicuticle & $\sim 12 \%$ & $\begin{array}{l}\text { 18-MEA lipid layer } \\
\text { attached to outer epicuticle } \\
\text { contributes to lubricity of } \\
\text { the hair }\end{array}$ \\
\hline A-layer & $\sim 30 \%$ & $\begin{array}{l}\text { Highly cross-linked } \\
\text { Mechanically tough } \\
\text { Chemically resilient }\end{array}$ \\
\hline Exocuticle & $\sim 15 \%$ & \\
\hline Endocuticle & $\sim 3 \%$ & $\begin{array}{l}\text { Lamellar structure } \\
\text { Consists of inner } \beta \text {-layer, } \delta- \\
\text { layer, and outer } \beta \text {-layer }\end{array}$ \\
\hline Inner layer & - & \\
\hline $\begin{array}{l}\text { Cell membrane complex } \\
\text { (CMC) }\end{array}$ & $\sim 2 \%$ &
\end{tabular}

Table 2.2 Various layers of the cuticle and their details 
The outer layer is the epicuticle, which is covered with a thin layer of covalently attached lipid 18-Methyl Eicosanoic Acid (18-MEA) (see Table 2.1). The A-layer is a component of high cystine content $(\sim 30 \%)$ and located on the outer-facing aspect of each cell. The A-layer is highly crosslinked which gives this layer considerable mechanical toughness and chemical resilience, and the swelling in water is presumed to be minimal. The exocuticle, which is immediately adjacent to the A-layer, is also of high cystine content $(\sim 15 \%)$. On the inner facing aspect of each cuticle cell is a thin layer of material which is known as the inner layer. Between the exocuticle and inner layer is the endocuticle which is low in cystine $(\sim 3 \%)$. The cell membrane complex itself is a lamellar structure, which consists of the inner $\beta$-layer, the $\delta$-layer and the outer $\beta$-layer.

Figure 2.2(a) shows SEM images of virgin Caucasian, Asian and African hair [Wei et al., 2005]. It can be seen that the Asian hair is the thickest (about $100 \mu \mathrm{m}$ ), followed by African hair (about $80 \mu \mathrm{m}$ ) and Caucasian hair (about $50 \mu \mathrm{m}$ ). The visible cuticle cell is about 5 to $10 \mu \mathrm{m}$ long for the three hair. A listing of various cross-section dimensional properties are presented in Table 2.3 [Wei et al., 2005]. While Caucasian and Asian hair typically have similar cross-sectional shape (Asian hair being the most cylindrical), African hair has a highly elliptical shape. African hair is much more curly and wavy along the hair fiber axis than Caucasian or Asian hair. Figure 2.2(b) shows the SEM images of virgin Caucasian hair at three locations: near scalp, middle and near tip. Three magnifications were used to show the significant differences. The hair near scalp had complete cuticles, while no cuticles were found on the hair near tip. This may be because that the hair near the tip experienced more mechanical damage during its life 
than the hair near the scalp. The hair in the middle experienced intermediate damage, i.e., one or more cuticle scales were worn away, but many cuticles stayed complete. If some substructures of one cuticle scale, like A-layer or A-layer and exocuticle (see Figure 2.1) are gone, or even worse, one or several cuticle scales are worn away, it is impossible to heal the hair biologically because hair fibers are composed of dead cells.

However, it is possible to physically "repair" the damaged hair by using conditioner, one of whose functions is to cover or fill the damaged area of the cuticles. Figure 2.2(c) shows the high magnification SEM images of virgin and treated Caucasian hair. The endocuticles (pointed by arrows) were found in both hair. In order for the conditioner to physically repair the hair, it is expected for it to cover the endocuticles. In the case of severely damaged hair, for example, an edge of one whole cuticle scale worn away, the conditioner may fill that damaged edge. In the SEM image of the treated hair in Figure 2.2(c), the substance which stayed near the cuticle edge is probably the conditioner (pointed by an arrow).

Figure 2.3 shows the AFM images of various virgin hair, along with the section plots [LaTorre and Bhushan, 2005a]. The arrows point to the position where the section plots were taken from. Each cuticle cell is nearly parallel to the underlying cuticle cell, and they all have similar angles to the hair axis, forming a shingle-like hair surface structure. The visible cuticle cell is approximately 0.3 to $0.5 \mu \mathrm{m}$ thick and about 5 to 10 $\mu \mathrm{m}$ long for all three hair. 

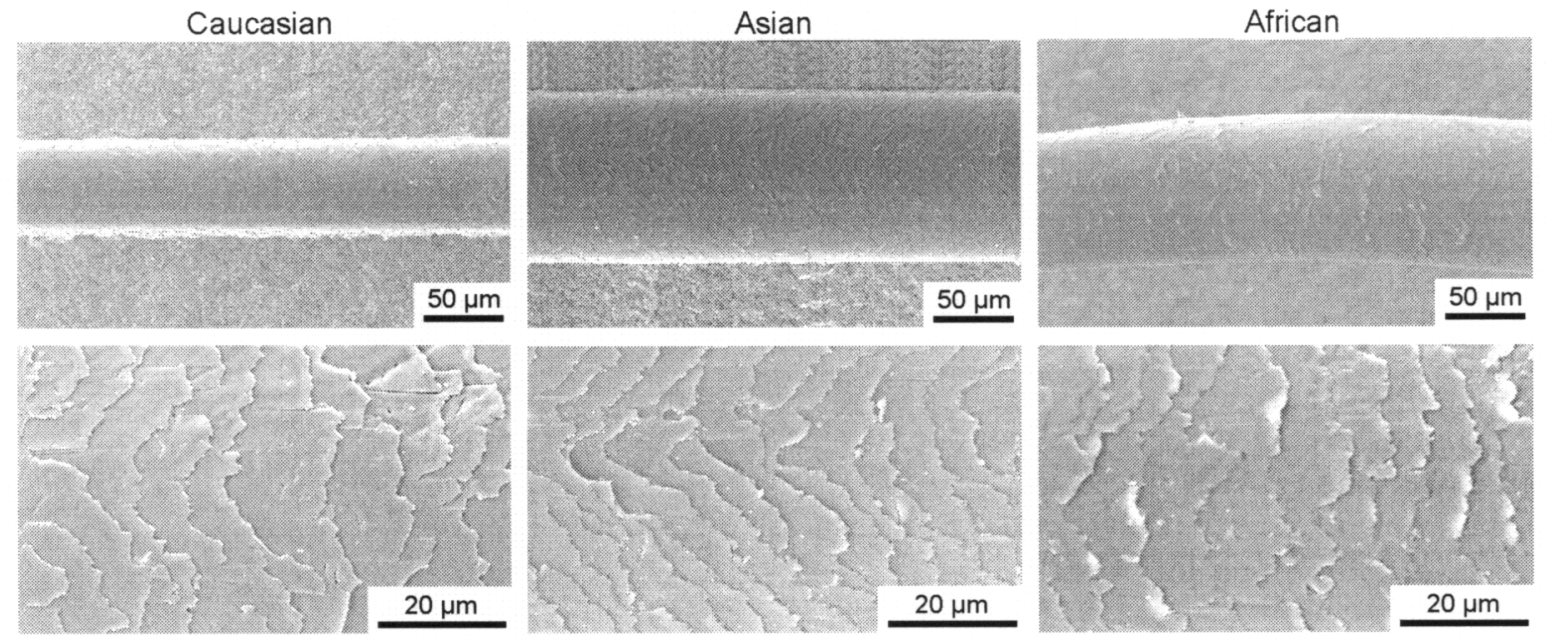

(a)

Continued

Fig. 2.2 (a) SEM images of various hair (b) SEM images of virgin Caucasian hair at three locations (c) SEM images of Caucasian, virgin and treated hair [Wei et al., 2005]. 
Fig. 2.2 continued

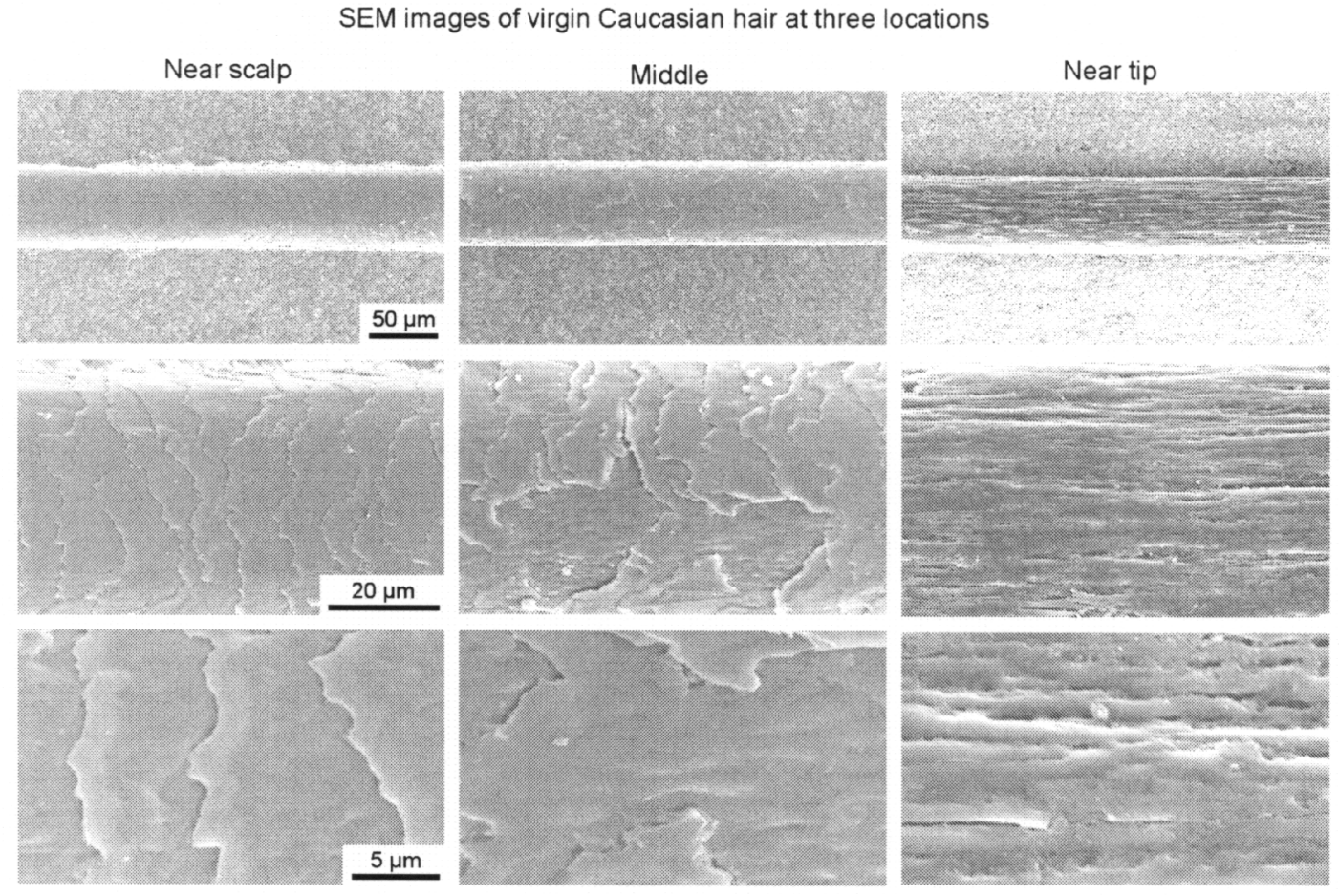

(b) 
Fig. 2.2 continued

SEM images of Caucasian, virgin and treated hair

Virgin

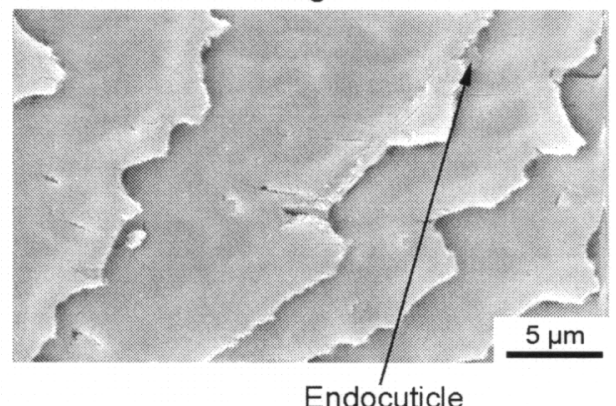

Endocuticle

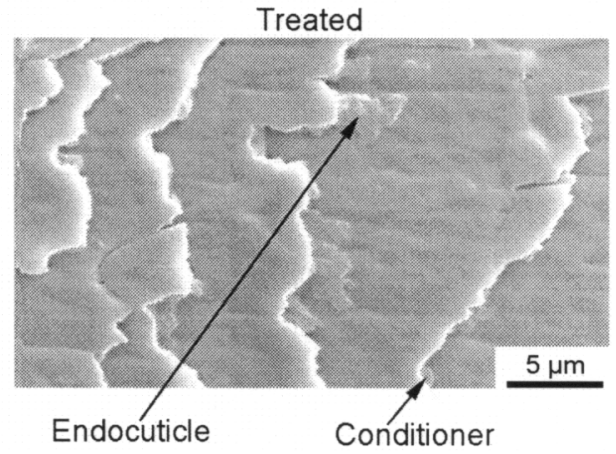

(c) 


\begin{tabular}{|c|c|c|c|c|c|c|}
\hline Shape & $\begin{array}{c}\text { Maximum } \\
\text { diameter } \\
\left(\mathrm{D}_{1}\right) \\
(\mu \mathrm{m})\end{array}$ & $\begin{array}{c}\text { Minimum } \\
\text { diameter } \\
\left(\mathrm{D}_{2}\right) \\
(\mu \mathrm{m})\end{array}$ & $\begin{array}{c}\text { Ratio } \\
\mathrm{D}_{1} / \mathrm{D}_{2}\end{array}$ & $\begin{array}{c}\text { Number of } \\
\text { cuticle } \\
\text { scales }\end{array}$ & $\begin{array}{c}\text { Cuticle } \\
\text { scale } \\
\text { thickness } \\
(\mu \mathrm{m})\end{array}$ \\
\hline Caucasian & Nearly oval & 74 & 47 & 1.6 & $6-7$ & $0.3-0.5$ \\
\hline Asian & Nearly round & 92 & 71 & 1.3 & $5-6$ & $0.3-0.5$ \\
\hline African & Oval-flat & 89 & 44 & 2.0 & $6-7$ & $0.3-0.5$ \\
\hline
\end{tabular}

Average length of visible cuticle scale: about 5 to $10 \mu \mathrm{m}$

Table 2.3 Variation in cross-sectional dimensions of human hair.

\subsubsection{The cortex and medulla}

The cortex contains cortical cells and the intercellular binding material, or the cell membrane complex. The cortical cells are generally 1 to $6 \mu \mathrm{m}$ thick and $100 \mu \mathrm{m}$ long, which run longitudinally along the hair fiber axis and take up the majority of the inner hair fiber composition [Randebrook, 1964]. The macrofibrils (about 0.1 to $0.4 \mu \mathrm{m}$ in diameter) comprise a major portion of the cortical cells. Each macrofibril consists of intermediate filaments (about $7.5 \mathrm{~nm}$ in diameter), previously called microfibrils, and the matrix. The intermediate filaments are low in cystine $(\sim 6 \%)$, and the matrix is rich in cystine $(\sim 21 \%)$. The cell membrane complex consists of cell membranes and adhesive material that binds the cuticle and cortical cells together. The intercellular cement of the cell membrane complex is primarily nonkeratinous protein, and is low in cystine content $(\sim 2 \%)$. The medulla of human hair, if present, generally makes up only a small percentage of the mass of the whole hair, and is believed to contribute negligibly to the mechanical properties of human hair fibers. 
Figure 2.4(a) shows the SEM images of virgin hair cross section [Wei et al., 2005] and Figure 2.4(b) shows the TEM images of a cross-section of human hair [Swift, 1997].

\subsubsection{Skin}

Skin covers and protects our bodies. The skin at the forehead and scalp areas are of most interest when dealing with human hair, since most of the hair care products are developed specifically for head hair. The skin of the hand and fingers is also of importance because the "feel" of hair is often sensed by physically touching the fibers with these regions. In general, skin is composed of three main parts: epidermis, dermis, and subcutaneous tissue (L'Oreal); see Figure 2.5.

The epidermis contains four distinct cellular layers: basal, spinous, granular, and horny. In the basal layer, melanocytes deliver the pigment melanin to keratinocytes. Keratinocyte cells that have been cornified are referred to as corneocytes [Pugliese, 1996]. Hexagonally shapped corneocyte cells compose the horny layer, or stratum corneum. The stratum corneum is the outer layer of the skin; at about $15-\mu \mathrm{m}$ thick, it acts as a mechanical, thermal, and chemical barrier from environmental factors and contamination. The complex organization of corneocytes and intercellular matrix contribute to the success of the barrier [Wertz and Downing, 1989]. In fact, Wertz et al. [1989] developed a structural model which observes the matrix as a lamellar phase composition of various lipids which provide a glue-like system to provide a barrier effect.

The dermis structure is known for its ability to handle most of the physical stresses imposed on the skin, and takes up roughly $90 \%$ of the mass [Pugliese, 1996]. The dermis is divided into an outermost papillary layer and underlying reticular layer. 
AFM images of various virgin hair
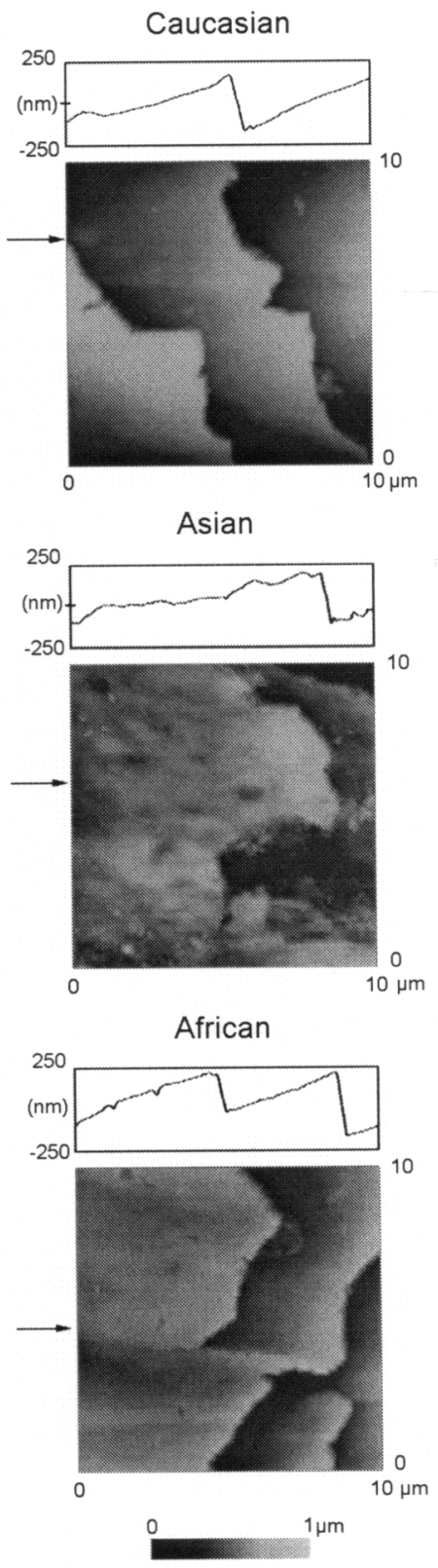

Fig. 2.3 AFM images of various virgin hair [LaTorre and Bhushan, 2005a]. 
SEM images of virgin hair cross section
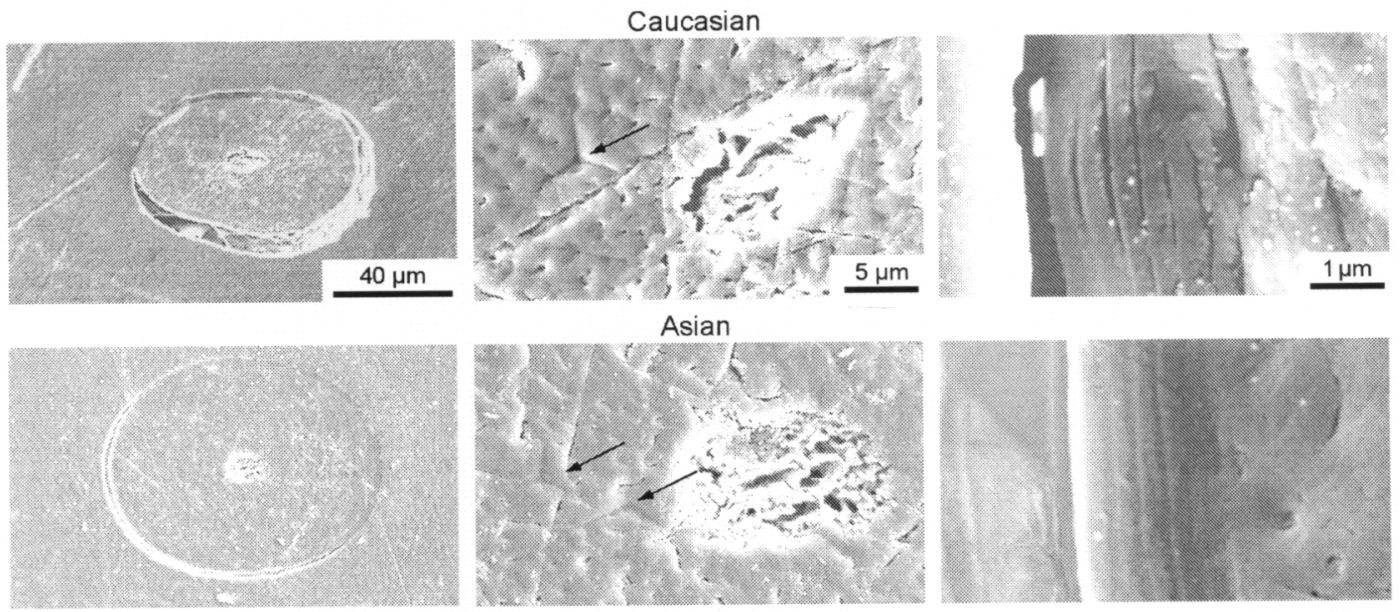

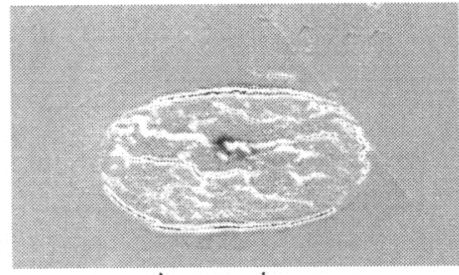

Larger view

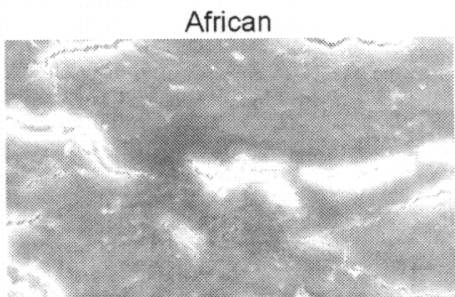

Cortex/Medulla region

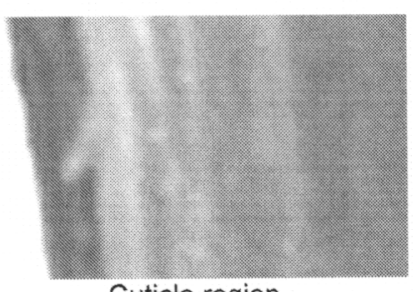

Cuticle region

(a)

Continued

Fig. 2.4 (a) SEM images of virgin hair cross-section (in the figure EXO, END, and CMC stand for exocuticle, endocuticle, and cell membrane complex, respectively) [Wei et al., 2005] (b) TEM of hair cross-section. 
Fig. 2.4 continued

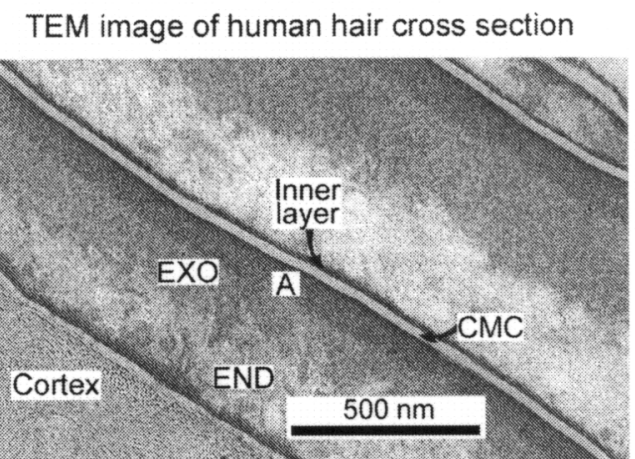

(b)

\subsection{Hair care: Cleaning and conditioning treatments, and damaging processes}

\subsubsection{Cleaning and conditioning treatments: Shampoo and Conditioner}

Shampoos are used primarily to clean the hair and scalp of dirt and other greasy residue that can build up after time. Shampoos also have many secondary functions including controlling dandruff, reducing irritation, and even conditioning. Conditioners, on the other hand, are used primarily to give the hair a soft, smooth feel which results in easier hair combing. Secondary functions include preventing "flyaway" hair due to static electricity, giving the hair a shiny appearance, and protecting the hair from further damage by forming a thin coating over the fibers.

Further developments in marketing and aesthetic factors (brand name, fragrance, feel, and color of the shampoos and conditioners) have created new market segments. In many instances, these factors have become primary reasons for use. 


\subsubsection{Shampoo: constitution and main functions}

The following discussion is based on Gray [2001, 2003]. As stated above, shampoos serve various cleaning functions for the hair and scalp. In the past, typical shampoos were mainly soap based products. However, soaps did not have very good lathering capability, and often left a residual "scum" layer on the hair that was undesirable and could not be rinsed off. In modern shampoos, advances in chemistry and technology have made it possible to replace the soap bases with complex formulas of cleansing agents, conditioning agents, functional additives, preservatives, aesthetic additives and even medically active ingredients. Table 2.4 shows the most common ingredients of shampoos and their functions.

\section{Cleansing agents}

In most modern shampoos, the primary cleansing agents are surfactants. Dirt and greasy residue are removed from the hair and scalp by these surfactants, making them the most important part of the shampoo. Surfactants have great lathering capabilities and rinse off very easily; see Table 2.4 for a full list of features.

Surfactant molecules have two different ends, one which is negatively charged and soluble in water (unable to mix with greasy matter), and another which is soluble in greasy matter (unable to mix with water). In general, surfactants clean the hair by the following process: Surfactant molecules encircle the greasy matter on the hair surface. The molecule end which is soluble in greasy matter buries itself in the grease, which leaves the water soluble molecule end to face outward with a negative charge. Since the hair fibers are negatively charged as well, the two negative charges repel each other. Thus, the greasy matter is easily removed from the hair surface and rinsed off. 


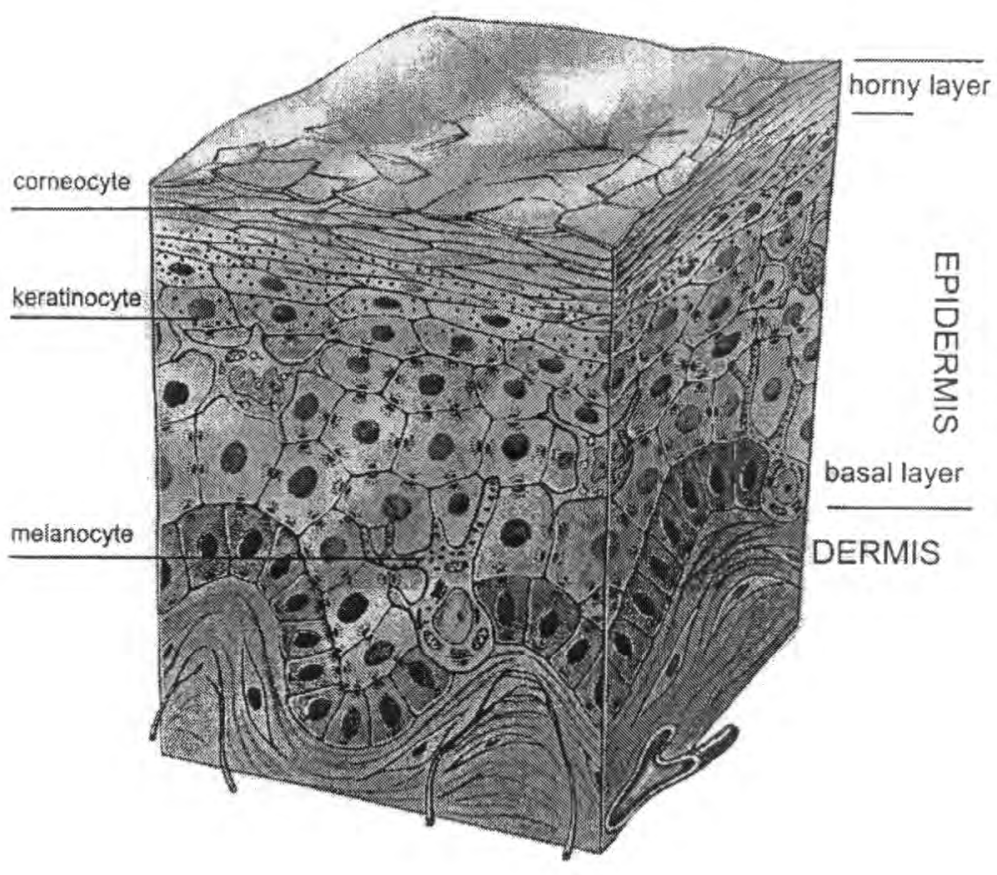

Fig. 2.5 Schematic image of human skin structure with different layers: dermis, epidermis, and horny layer (L'Oreal). 


\begin{tabular}{|l|l|}
\hline Shampoo component & Functions \\
\hline Cleansing agents & $\begin{array}{l}\text {-Produce lather to trap greasy matter, and prevent re- } \\
\text { deposition } \\
\text {-Remove dirt and grease from hair and scalp } \\
\text {-Stabilize the mixture and help keep the ingredient } \\
\text { network together } \\
\text {-Thicken the shampoo to the desired viscosity }\end{array}$ \\
\hline Conditioning agents & Condition the hair \\
\hline Functional additives & Control the viscosity and pH levels of the shampoo \\
\hline Preservatives & Prevent decomposition and contamination of shampoo \\
\hline Aesthetic additives & Enhance color, scent, and luminescence of shampoo \\
\hline Medically active ingredients & Aid treatment of dandruff or hair loss \\
\hline
\end{tabular}

Table 2.4 Components of common shampoos and their functions

Shampoos contain several surfactants, generally up to four, which clean the hair differently depending on the hair type of the individual. Mild cleansing systems, which do not damage or irritate the scalp, hair, and eyes, are now quite common. Conditioning agents

Many shampoos contain conditioning agents which serve many of the same roles as full conditioners. Conditioning agents are further described in the following subsection.

Functional additives

Functional additives can aid in controlling the thickness and feel of the shampoo itself. Simply stated, the right blend is required so that the shampoo is not too thin and not too thick. Functional additives can also control the acidity of the shampoo by obtaining a goal $\mathrm{pH}$ level, typically around a value of 4 . 


\section{Preservatives}

Preservatives detract germs and prevent decomposition of the shampoos. They also prevent various other health risks that accompany contamination by germs and bacteria. Typical preservatives in shampoos are sodium benzoate, parabens, DMDM hydantoin and tetrasodium EDTA.

\section{Aesthetic additives}

Shampoos contain many aesthetic additives which enhance appearance, color, and smell of the mixture. These additives typically give the shampoo the luminous shine and pleasant fragrance to which many consumers are accustomed.

\section{Medically active ingredients}

For people with dandruff and other more serious hair and scalp disorders, shampoos are available with active ingredients which aim to treat or control these conditions. In the treatment of dandruff, zinc pyrithione is a common shampoo additive. For hair loss issues, panthenol is commonly added to shampoos to aid in hair growth and moisture content.

\subsubsection{Conditioner: constitution and main functions}

As stated earlier, many shampoos have certain levels of conditioning agents which mimic the functions of a full conditioner product. Conditioner molecules contain cationic surfactants which give a positive electrical charge to the conditioner. The negative charge of the hair is attracted to the positively charged conditioner molecules, which results in conditioner deposition on the hair; see Figure 2.6. This is especially true for damaged hair, since damaging processes result in hair fibers being even more negatively charged. The attraction of the conditioner to hair results in a reduction of 
static electricity on the fiber surfaces, and consequently a reduction in the "fly away" behavior. The conditioner layer also flattens the cuticle scales against each other, which improves shine and color. The smooth feel resulting from conditioner use gives easier combing and detangling in both wet and dry conditions (see Table 1.2).

Conditioner consists of a gel network chassis (cationic surfactants, fatty alcohols, and water) for superior wet feel and combination of conditioning actives (cationic surfactants, fatty alcohols, and silicones) for superior dry feel. Figure 2.7 shows the transformation of the cationic surfactants and fatty alcohol mixture into the resulting gel network, which is a frozen lamellar liquid crystal gel phase. The process starts as an emulsion of the surfactants and alcohols in water. The materials then go through a strictly controlled heating and cooling process: the application of heat causes the solid compounds to melt, and the solidification process enables a setting of the lamellar assembly molecules in a fully extended conformation, creating a lamellar gel network. When this network interacts with the hair surface, the high concentration of fatty alcohols make them the most deposited ingredient group, followed by the silicones and cationic surfactants. Typical deposition levels for cationic surfactant, fatty alcohol, and silicone are around 500-800 ppm, 1000-2000 ppm, and $200 \mathrm{ppm}$, respectively. Typical concentrations are approximately $2-5$ weight $\%, 5-10$ weight $\%$, and $1-10$ weight $\%$, respectively [LaTorre et al., 2006]. 
Virgin or damaged hair

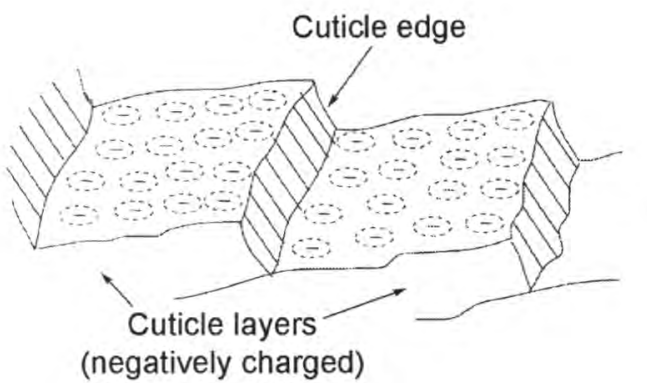

Treated hair

Conditioner deposits (positively charged)

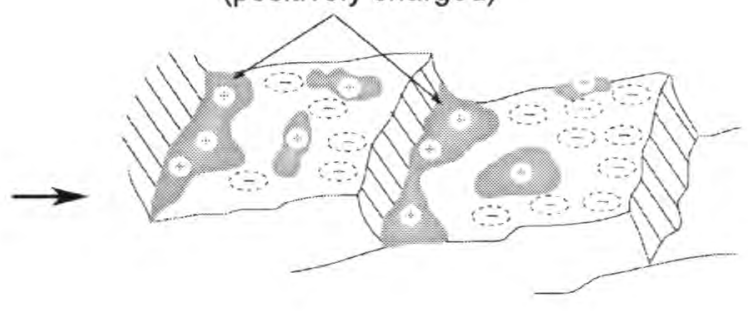

Fig. 2.6 Negatively charged hair and the deposition of positively charged conditioner on the cuticle surface [LaTorre et al., 2006]. 


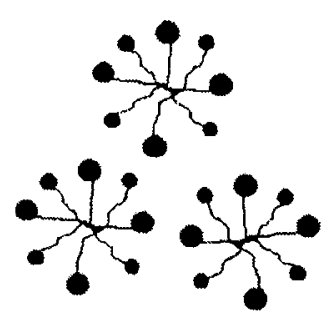

Oil / water emulsion

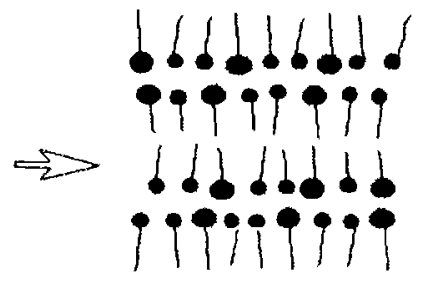

Lamellar liquid crystal

Surfactant Fatty alcohol

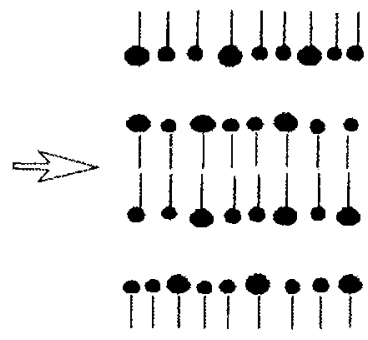

Gel network

Fig. 2.7 Conditioner formation from emulsion to gel network.

\begin{tabular}{|cc|}
\hline \multicolumn{2}{|c|}{ Gel network chassis for superior wet feel } \\
\hline Key Ingredients & Benefits \\
-Cationic surfactant & -Creamy texture \\
-Fatty alcohols & -Ease of spreading \\
-Water & -Slippery applying feel \\
& -Soft rinsing feel \\
\hline Combination of "conditioning actives" for superior dry feel \\
\hline Key Ingredients & Benefits \\
\hline -Silicones & -Moist \\
-Fatty alcohols & -Soft \\
-Cationic surfactant & -Dry combing ease \\
\hline
\end{tabular}

Table 2.5 Combinations of conditioner ingredients and their benefits towards wet and dry feel. 
The benefits of the conditioner are shown in Table 2.5 [LaTorre et al., 2006]. The wet feel benefits are creamy texture, ease of spreading, slippery feel while applying, and soft rinsing feel. The dry feel benefits are moistness, softness, and easier dry combing. Each of the primary conditioner ingredients also have specific functions and roles that affect performance of the entire product.

Table 2.6 displays the functions of the major conditioner ingredients and their chemical structure [LaTorre et al., 2006]. Cationic surfactants are critical to the forming of the lamellar gel network in conditioner, and also act as a lubricant and static control agent, since their positive charge aids in counteracting the negative charge of the hair fibers. Fatty alcohols are used to lubricate and moisturize the hair surface, along with forming the gel network. Finally, silicones are the main source of lubrication in the conditioner formulation. 


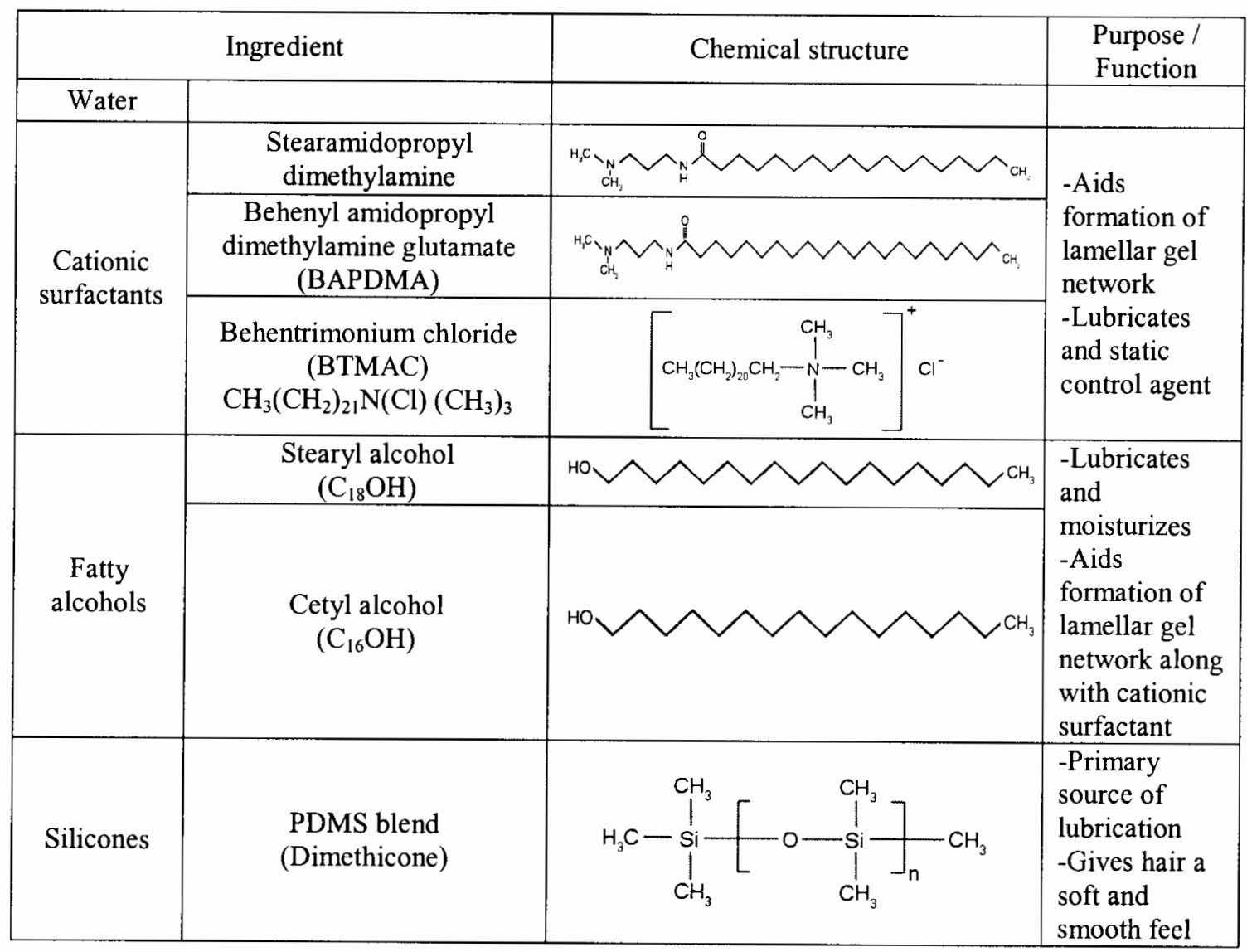

Table 2.6 Chemical structure and purpose/function of conditioner ingredients 


\subsubsection{Damaging processes}

In Section 2.2.1 we discussed some of the products which aid in "treating" the hair. There are other hair care products and processes which, while creating a desired look or style to the hair, also bring about significant damage to the fibers (see Table 1.1). Most of these processes occur on some type of periodic schedule, whether it be daily (combing the hair), or monthly (haircut and coloring at a salon). In general, hair fiber damage occurs most readily by mechanical or chemical means, or by a combination of both (chemo-mechanical).

\section{Mechanical damage}

Mechanical damage occurs on a daily basis for many individuals. The damage results from large physical forces or temperatures which degrade and wear the outer cuticle layers. Common causes are

- combing (generally with plastic objects, and often multiple times over the same area which leads to scratching and wearing of the cuticle layers)

- $\quad$ scratching (usually with fingernails around the scalp)

- cutting (affects the areas surrounding the fiber tips)

- blowdrying (high temperatures thermally degrade the surface of the hair fibers)

\section{Permanent wave treatment}

Permanent wave treatments saw many advances in the beginning of the $20^{\text {th }}$ century, but have not changed much with the invention of the Cold Wave around the turn of that century. Generally speaking, the Cold Wave uses mercaptans (typically thioglycolic acid) to break down disulphide bridges and style the hair without much user 
interaction (at least in the period soon after the perm application) [Gray, 2001]. The Cold Wave process does not need increased temperatures (no thermal damage to the hair), but generally consists of a reduction period (whereby molecular reorientation to the cuticle and cortex occurs via a disulfide-mercaptan interchange pathway [Robbins, 1994]) followed by rinsing, setting of the hair to the desired style, and finally neutralization to decrease the mercaptan levels and stabilize the style. The chemical damage brought on by the permanent wave can increase dramatically when not performed with care.

\section{Chemical relaxation}

Commonly used as a means of straightening hair (especially in highly curved, tightly curled African hair), this procedure uses an alkaline agent, an oil phase, and a water phase of a high viscosity emulsion to relax and reform bonds in extremely curly hair. A large part of the ability to sculpt the hair to a desired straightness comes from the breakage of disulfide bonds of the fibers.

Coloring and dyeing

Hair coloring and dyeing have become extremely successful hair care procedures, due in part to "over-the-counter" style kits which allow home hair care without professional assistance. The most common dyes are para dyes, which contain paraphenylenediamine (PPD) solutions accompanied by conditioners and antioxidants. Hydrogen peroxide $\left(\mathrm{H}_{2} \mathrm{O}_{2}\right)$ is combined with the para dyes to effectively create tinted, insoluble molecules which are contained within the cortex and are not small enough to pass through the cuticle layers, leaving a desired color to the hair. Due to the levels of hydrogen peroxide, severe chemical damage can ensue in the cuticle and cortex.

\section{Bleaching}


Like dyeing, bleaching consists of using hydrogen peroxide to tint the hair.

However, bleaching can only lighten the shade of hair color, as the $\mathrm{H}_{2} \mathrm{O}_{2}$ releases oxygen to bind hair pigments [Gray, 2001]. Bleaching may also be applied to limited areas of the hair (such as in highlights) to create a desired look. The chemical damage brought on by bleaching leads to high porosity and severe wear of the cuticle layer. 


\section{CHAPTER 3}

\section{EXPERIMENTAL TECHNIQUES}

To date, most information about the detailed structure of human hair was obtained from scanning electron microscope (SEM) and transmission electron microscope (TEM) observations [Swift, 1991; Robbins, 1994; Swift, 1997; Wei et al., 2005]. Table 3.1 shows a comparison between the various tools used to study hair on the micro/nanoscale. The SEM has long been the standard means of investigating the surface topography of human hair. The SEM uses an electron beam to give a 'photographic' image of the sample, but cannot provide quantitative data regarding the surface. SEM requires the hair sample to be covered with a very thin layer of a conductive material and needs to be operated under vacuum during both metallization and measurements. Surface metallization and vacuum exposure could potentially induce modifications to the surface details. Figure 2.4(a) and (b) show typical images of human hair obtained by SEM and TEM, respectively. TEM examinations provide fine detailed internal structure of human hair. However, thin sections of 50-100 nm thickness and heavy metal compounds staining treatment are required for TEM examinations. The cutting of these thin sections with the aid of an ultra-microtome is not an easy task. Moreover, since both SEM and TEM techniques cannot measure the physical properties (mechanical, tribological, etc.) of 
various cellular structure of human hair of interest and do not allow ambient imaging conditions, many outstanding issues remain to be answered.

\begin{tabular}{|c|c|c|c|c|c|}
\hline Method & $\begin{array}{c}\text { Type of } \\
\text { information }\end{array}$ & $\begin{array}{c}\text { Quantitative } \\
\text { data }\end{array}$ & $\begin{array}{c}\text { Normal } \\
\text { load }\end{array}$ & $\begin{array}{c}\text { Resolution } \\
(\mathrm{nm})\end{array}$ & Limitations \\
\hline $\begin{array}{l}\text { Scanning } \\
\text { electron } \\
\text { microscopy } \\
\text { (SEM) }\end{array}$ & Structural & $\begin{array}{l}\text { Gross } \\
\text { dimensions }\end{array}$ & None & $\begin{array}{l}0.2-1 \\
\text { (spatial) }\end{array}$ & $\begin{array}{l}\text {-Requires thin } \\
\text { conductive coating on } \\
\text { sample } \\
\text {-Requires vacuum } \\
\text { environment } \\
\text {-Expensive } \\
\text { instrumentation } \\
\text {-Tedious }\end{array}$ \\
\hline $\begin{array}{l}\text { Transmission } \\
\text { electron } \\
\text { microscopy } \\
\text { (TEM) }\end{array}$ & Structural & $\begin{array}{l}\text { Gross } \\
\text { dimensions }\end{array}$ & None & $\begin{array}{l}0.2-1 \\
\text { (spatial) }\end{array}$ & $\begin{array}{l}\text {-Requires thin sections } \\
(<100 \mathrm{~nm}) \text { and heavy } \\
\text { metal compound } \\
\text { staining treatment } \\
\text {-Requires vacuum } \\
\text { environment } \\
\text {-Expensive } \\
\text { instrumentation } \\
\text {-Tedious }^{\mathrm{a}}\end{array}$ \\
\hline $\begin{array}{l}\text { Atomic } \\
\text { force/friction } \\
\text { force } \\
\text { microscopy } \\
\text { (AFM/FFM) }\end{array}$ & $\begin{array}{l}\text { Structural } \\
\text { Mechanical } \\
\text { Tribological }\end{array}$ & $\begin{array}{l}\text {-Surface } \\
\text { roughness } \\
\text {-Elastic modulus } \\
\text {-Viscoelastic } \\
\text { properties } \\
\text {-Friction } \\
\text {-Adhesion } \\
\text {-Conditioner } \\
\text { thickness }\end{array}$ & $\begin{array}{l}<0.1 \mathrm{nN} \\
-500 \mathrm{nN}^{\mathrm{a}}\end{array}$ & $\begin{array}{l}0.2-1 \\
(\text { spatial) } \\
0.02 \\
\text { (vertical) }^{\mathrm{a}}\end{array}$ & None \\
\hline
\end{tabular}

${ }^{\mathrm{a}}$ Bhushan [2002]

Table 3.1 Comparison of methods used to characterize hair on micro/nanoscale 
AFM has been commonly used for characterization of tribological and mechanical properties of surfaces [Bhushan, 1999a, b, 2002, 2004, 2005]. As a non-invasive technique, AFM can evaluate the effect of sample treatment since it can be used without requiring any specific treatment. Most importantly, it can be operated in ambient conditions in order to study the effect of environment. AFM provides the potential for being able to see the cellular structure and molecular assembly of hair, for determining various properties of hair, such as friction, adhesion, wear, elastic stiffness and viscoelastic properties, and for investigating the physical behavior of various cellular structure of hair in various environments. To date, most of AFM studies on hair fibers were focused on the surface topographic imaging [Smith and Swift, 2002; LaTorre and Bhushan, 2005a] and friction, adhesion and wear properties [LaTorre and Bhushan, 2005a, b; LaTorre et al., 2006].

A schematic of an AFM imaging a hair fiber is shown in Figure 3.1. AFM/FFM uses a sharp tip with a radius of approximately $30-50 \mathrm{~nm}$. This significant reduction in tip-to-sample interaction compared to the macroscale allows the simulation of single asperity contact to give detailed surface information. While there are several AFM operating modes, so called "contact mode" is most relevant for studying tribological properties because it allows simultaneous measurement of surface roughness and friction force.

When skin comes in contact with hair, actual contact occurs over a large number of asperities. During relative motion, friction and adhesion are governed by the surface interactions which occur at these asperities. To date, much of the work in the industry has focused on the measurement of macroscale friction, particularly between a skin 
replica and a hair switch of interest [Robbins, 1994]. Figure 3.2 shows schematics of typical macro- and nanoscale test apparatuses.

Schematic diagram of AFM

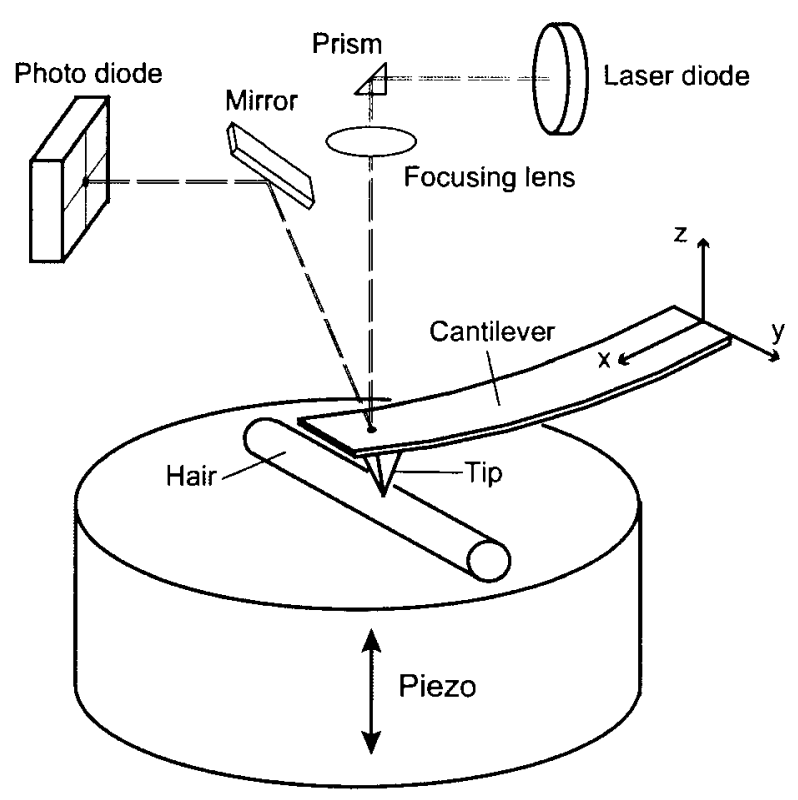

Fig. 3.1 Schematic diagram of AFM operation with human hair sample. 
Friction test apparatuses

at macro- and micro/nanoscales
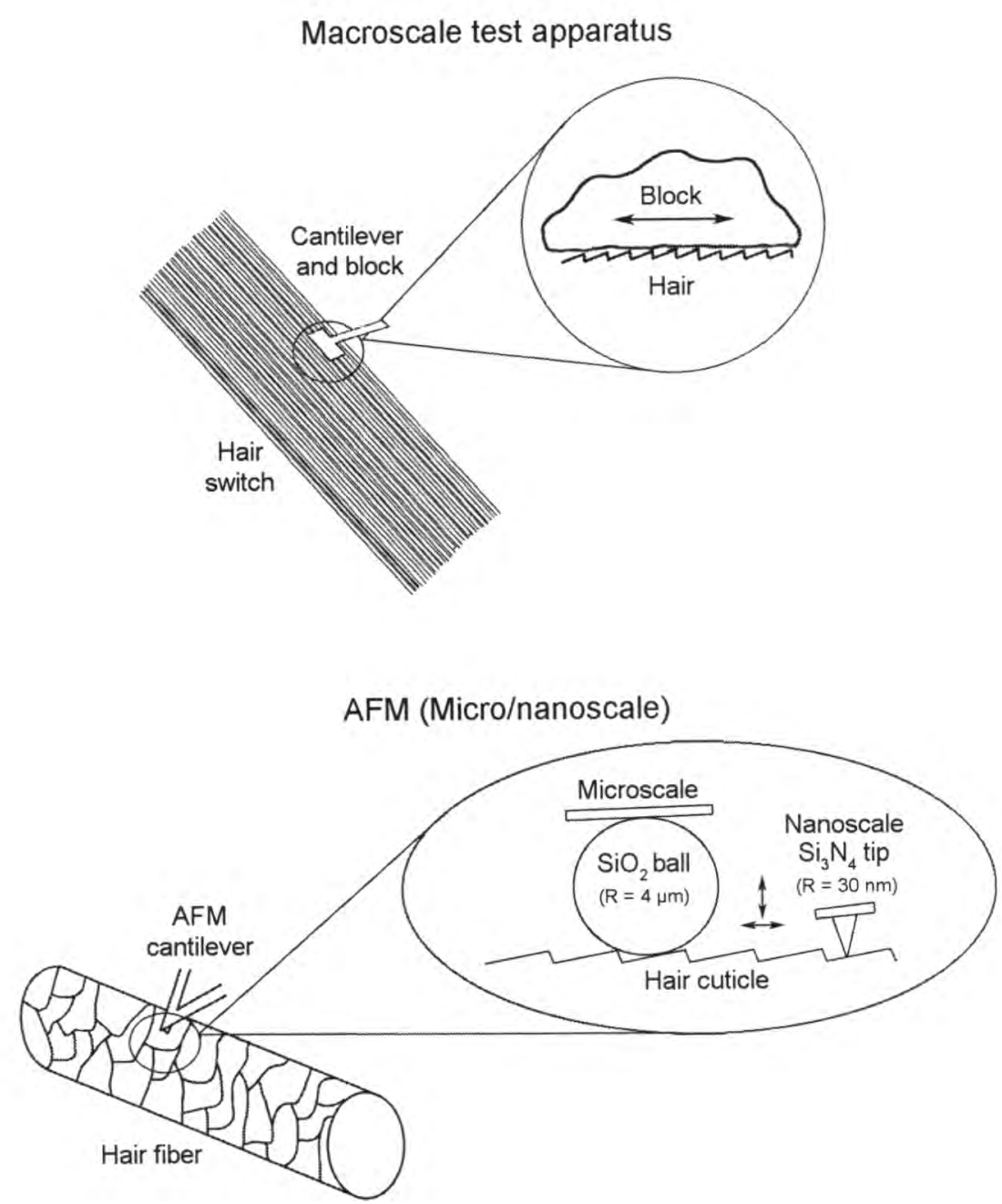

Fig. 3.2 Comparison of macroscale and micro/nanoscale friction test apparatuses. 


\subsection{Experimental procedure}

\subsubsection{Specimen mounting}

Hair specimens were mounted onto AFM sample pucks using Liquid Paper ${ }^{\circledR}$ correction fluid. A thin layer of the fluid was brushed onto the puck, and when the fluid hardened into a tacky state, the hair sample was carefully placed. The Liquid Paper ${ }^{\circledR}$ dries quickly to keep the hair firmly in place. An optical microscope was used to preliminarily image the specimen to ensure none of the Liquid Paper ${ }^{\circledR}$ was deposited on the hair surface.

Synthetic materials were attached to AFM sample pucks using double-sided adhesive tape.

\subsubsection{Nanotribological property measurements}

Surface roughness, friction force, and adhesive force measurements were performed using a commercial AFM system (MultiMode Nanoscope IIIa, Digital Instruments, Santa Barbara, $\mathrm{CA})$ in ambient conditions $\left(22 \pm 1^{\circ} \mathrm{C}, 50 \pm 5 \%\right.$ relative humidity) [LaTorre and Bhushan, 2005a, b; LaTorre et al., 2006]. Square pyramidal $\mathrm{Si}_{3} \mathrm{~N}_{4}$ tips of nominal $30-50 \mathrm{~nm}$ radius attached to the end of a soft $\mathrm{Si}_{3} \mathrm{~N}_{4}$ cantilever beam (spring constant of $0.06 \mathrm{~N} / \mathrm{m}$ ) were used for surface roughness and nanoscale friction force measurements. This softer cantilever was used to minimize damage to the hair. After engagement of the tip with the cuticle surface, the tip was scanned perpendicular to the longitudinal axis of the fiber. The tip was centered over the cross section in order to be at the very top of the fiber, so as to negate effects caused by the AFM tip hitting the sides of the hair and adding error to the measurements. In order to minimize scanning artifacts, a scan rate of $1 \mathrm{~Hz}$ was used for all measurements. Topographical images to 
characterize the shape and structure of the various hair were taken at $5 \times 5,10 \times 10$, and $20 \times 20 \mu \mathrm{m}^{2}$ scans at a normal load of $5 \mathrm{nN}$. These scan sizes were suitable for capturing the surface features of multiple scales and scale edges of the cuticle. To characterize roughness, $2 \times 2 \mu \mathrm{m}^{2}$ scans of the cuticle surface without edges were used. Surface roughness images shown in this thesis were processed using a first-order "planefit" command available in the AFM software, which eliminates tilt in the image. Roughness data as well as friction force data were taken after the "planefit" command was employed. A first-order "flatten" command was also used on friction force mappings to eliminate scanning artifacts and present a cleaner image.

Friction force mapping of the scan area was collected simultaneously with roughness mapping. Figure 3.2 (bottom cartoon) and Figure 3.3 show the AFM tip scanning over the hair surface for untreated and conditioner treated hair, respectively. The effects of the conditioner can be examined by comparing the friction information. A quantitative measure of friction force was calculated by first calibrating the force based on a method by Bhushan [Bhushan, 1999a, 2002]. The normal load was varied from $5 \mathrm{nN}$ to $45 \mathrm{nN}$ in roughly $5 \mathrm{nN}$ increments, and a friction force measurement was taken at each increment. By plotting the friction force as a function of normal force, the average coefficient of friction was determined from the slope of the least squares fit line of the data.

Adhesive force measurements were made with square pyramidal $\mathrm{Si}_{3} \mathrm{~N}_{4}$ tips attached to the end of a $\mathrm{Si}_{3} \mathrm{~N}_{4}$ cantilever beam (spring constant of $0.58 \mathrm{~N} / \mathrm{m}$ ), using the "force calibration plot technique". In this technique, the AFM tip is brought into contact with the sample by extending the piezo vertically, then retracting the piezo and 
Conditioner on cuticle of treated hair and interaction with AFM tip

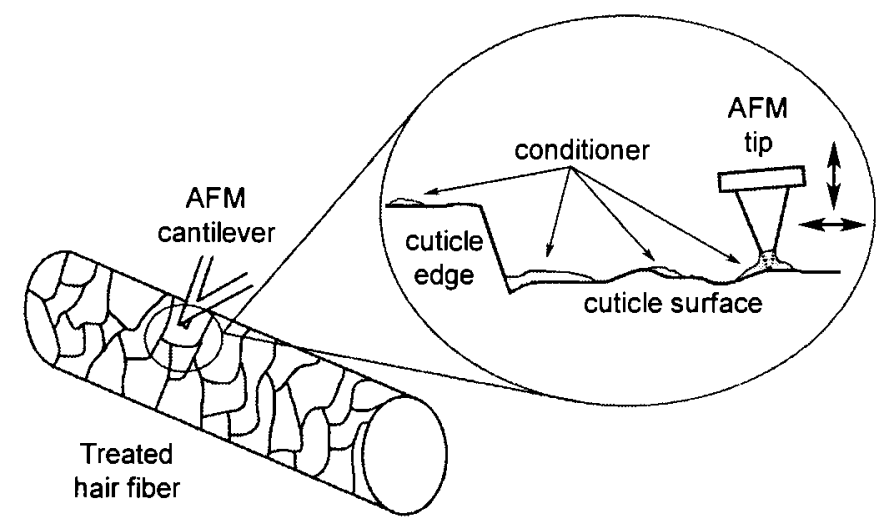

Fig. 3.3 Interactions between the AFM tip and conditioner on the cuticle surface for treated hair [LaTorre and Bhushan, 2005b]. 
calculating the force required to separate the tip from the sample. The method is described in detail by Bhushan [Bhushan, 1999a, b, 2002, 2004].

The cantilever deflection is plotted on the vertical axis against the Z-position of the piezo scanner in a force calibration plot, Figure 3.4. As the piezo extends (from right to left in the graph), it approaches the tip and does not show any deflection while in free air. The tip then approaches within a few nanometers (point A) and becomes attached to the sample via attractive forces at very close range (point B). After initial contact, any extension of the piezo results in a further deflection of the tip. Once the piezo reaches the end of its designated ramp size, it retracts to its starting position (from left to right). The tip goes beyond zero deflection and enters the adhesive regime. At point $\mathrm{C}$, the elastic force of the cantilever becomes equivalent to the adhesive force, causing the cantilever to snap back to point $\mathrm{D}$. The horizontal distance between $\mathrm{A}$ and $\mathrm{D}$ multiplied by the stiffness of the cantilever results in a quantitative measure of adhesive force [Bhushan, 1999a, 1999b, 2002, 2005].

The force calibration plot allows for the calculation of an adhesive force at a distinct point on the sample surface. Consequently, by taking a force calibration plot at discrete sampling intervals over an entire scan area, a resulting adhesive force mapping (also known as force-volume mapping) can be created to display the variation in adhesive force over the surface [Bhushan and Dandavate, 2000]. In this work, plots were taken at $64 \times 64$ distinct points over a scan area of $2 \times 2 \mu \mathrm{m}^{2}$ for all hair types and ethnicities. Current Digital Instruments software for the Multimode AFM does not allow direct calculation of the adhesive force from the force-volume maps. Thus, a custom program coded in Matlab was used to display the force-volume maps. The adhesive force for each 


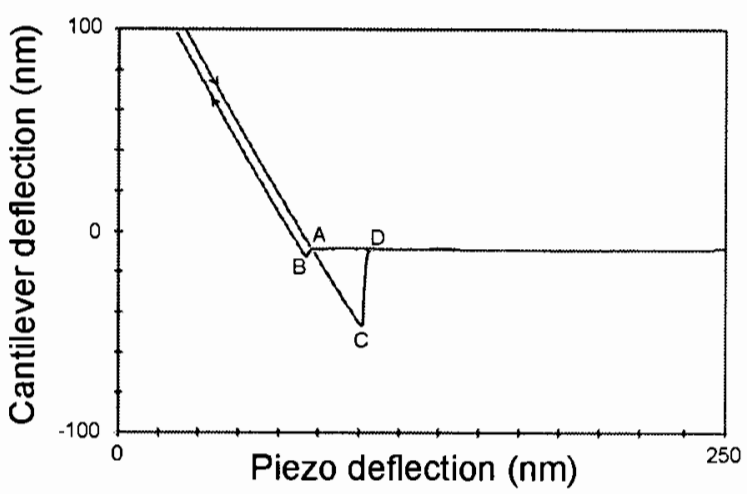

Fig. 3.4 Typical force calibration plot for Caucasian virgin hair. Contact between the tip and hair occurs at point $\mathrm{B}$. At point $\mathrm{C}$, the elastic force of the cantilever becomes equivalent to the adhesive force, causing the cantilever to snap back to point $\mathrm{D}$.

force calibration plot was obtained by multiplying the spring constant with the horizontal distance (in the retract mode) traveled by the piezo from the point of zero applied load to the point where the tip snaps off.

\subsubsection{Relative humidity, temperature, and soaking, and durability measurements}

A humidity-temperature detector was used to monitor the humidity inside a Plexiglas test chamber enclosing the AFM system. An experimental setup was used to control the humidity inside the chamber. Measurements were taken at nominal relative humidities of 5, 50, and $80 \%$. Hair fibers were placed at each humidity for approximately 2 hours prior to measurements. 
A homemade thermal stage was used to conduct temperature effect measurements at $20,37,50$, and $80{ }^{\circ} \mathrm{C}$. Hair fibers were exposed to each temperature condition for approximately 30 minutes prior to testing.

Soak tests were performed as follows: A dry hair fiber was taken from a switch, and a sample was cut from the fiber (approximately $7 \mathrm{~mm}$ long) for coefficient of friction measurements. An adjacent sample was also taken from the fiber and placed in a small beaker filled with de-ionized water. The sample was subjected to the aqueous environment for 5 minutes, which is representative of a typical exposure time when showering/bathing, then immediately analyzed with AFM. It should be noted that hair becomes saturated when wet in about 1 minute and remains saturated if kept in a humid environment. It was determined from unpublished results that if the wet hair was exposed to the ambient environment for more than 20 minutes while in the AFM, the hair became dry and coefficient of friction became similar to that of dry hair. Thus, coefficient of friction measurements were made within a 20 minute time frame for each sample.

In order to simulate scratching that can occur on the surface of the hair and its effect on the friction force on the cuticle surface, a durability test was conducted using a stiff silicon tip (spring constant of $40 \mathrm{~N} / \mathrm{m}$ ). A load of $10 \mu \mathrm{N}$ was used on a $2 \mu \mathrm{m}$ section of the cuticle. A total of 1000 cycles were performed at $2 \mathrm{~Hz}$. Measurements were conducted using an AFM. Friction force signal was recorded with respect to cycling time.

\subsubsection{Microscale and macroscale friction and adhesion measurements for scale} effects study

Microscale coefficient of friction and adhesive force measurements were performed using a commercial AFM system (MultiMode Nanoscope IIIa, Digital 
Instruments, Santa Barbara, CA) in ambient conditions $\left(22 \pm 1^{\circ} \mathrm{C}, 50 \pm 5 \%\right.$ relative humidity). For microscale data, a $4 \mu \mathrm{m}$ radius silica ball was mounted on a $\mathrm{Si}_{3} \mathrm{~N}_{4}$ cantilever (spring constant of $0.58 \mathrm{~N} / \mathrm{m}$ ). A quantitative measure of friction force was calculated as described previously, except that the normal load was varied over a larger range (0-180 $\mathrm{nN}$ with microscale tip, compared to $0-45 \mathrm{nN}$ with nanoscale tip). Adhesive force measurements were made with the microscale AFM tips using the "force calibration plot technique", as described previously.

For macroscale coefficient of friction, the tests were conducted using a flat-on-flat tribometer under reciprocating motion, with measurement techniques similar to those described in Bhushan et al. [2005]. Macroscale coefficient of friction data for virgin and virgin treated hair were taken from Bhushan et al. [2005]. The values for other hair were taken in part from the indexed coefficient of friction values presented in LaTorre and Bhushan [2005b] which were transformed into actual values by the following procedure. A comparison was made between nanoscale data for chemo-mechanically damaged and chemically damaged hair, and a $15 \%$ difference was found. The same $\%$ difference was assumed on the macroscale, such that the value for chemo-mechanically damaged hair presented in Bhushan et al. [2005] was used to calculate a value for the chemically damaged hair coefficient of friction. From there, the indexed values for the chemically damaged treated ( 1 cycle and 3 cycle) hair were scaled based on the newly found chemically damaged hair value.

\subsection{Hair and skin samples}

For the research reported in this chapter, all hair samples were received from Procter \& Gamble (Cincinnati, OH) and Procter \& Gamble Far East (Kobe, Japan) and 
prepared per Appendix A. Samples arrived as hair switches approximately $0.3 \mathrm{~m}$ long. Although the exact location from the root is unknown, it is estimated that hair samples used for testing were between 0.1 to $0.2 \mathrm{~m}$ from the scalp.

Table 3.2 presents a list of all samples used. The main hair categories of interest are: virgin (untreated), virgin (treated with 1 cycle of commercial conditioner), chemomechanically damaged (untreated), chemically damaged (untreated), and chemically damaged (treated with 1 or 3 cycles of commercial conditioner or matrix of conditioner ingredients). Virgin samples are considered to be baseline specimens and are defined as having an intact cuticle and absence of chemical damage. Chemo-mechanically damaged hair fibers have been exposed to one or more cycles of coloring and permanent wave treatment, washing, and drying, as well as combing (to contribute mechanical damage) which are representative of common hair management and alteration. In the case of African damaged hair samples, chemical damage occurred only by chemical straightening. Chemically damaged fibers have not been exposed to the combing sequence in their preparation. All treated hair samples were treated with either one or three rinse/wash cycles of a conditioner similar to a Procter \& Gamble commercial product, or were treated with various combinations of surfactant, fatty alcohol, and silicone types and deposition levels, presented in the matrix of Table 3.3 [LaTorre et al., 2006]. Two different types of cationic surfactants were used: behentrimonium chloride (BTMAC) and behenyl amidopropyl dimethylamine (BAPDMA). Only one group of fatty alcohols were used for all samples. In the last set of samples, two different silicones were used: a PDMS (blend with high MW) silicone and an amino silicone. 


\begin{tabular}{|c|l|}
\hline \multicolumn{1}{|c|}{ Hair and Skin Samples } \\
\hline Sample & \multicolumn{1}{c|}{ Type } \\
\hline $\begin{array}{c}\text { Caucasian } \\
\text { hair }\end{array}$ & $\begin{array}{l}\text {-Virgin } \\
\text {-Virgin, treated (1 cycle commercial conditioner) } \\
\text {-Chemo-mechanically damaged } \\
\text {-Chemically damaged } \\
\text {-Chemically damaged, treated (1 cycle commercial conditioner) } \\
\text {-Chemically damaged, treated (3 cycles commercial conditioner) } \\
\text {-Chemically damaged, treated (various ingredients, see Table 3.3) }\end{array}$ \\
\hline Asian hair & $\begin{array}{l}\text {-Virgin } \\
\text {-Virgin, treated (1 cycle commercial conditioner) } \\
\text {-Chemo-mechanically damaged }\end{array}$ \\
\hline African hair & $\begin{array}{l}\text {-Virgin } \\
\text {-Virgin, treated (1 cycle commercial conditioner) } \\
\text {.Chemo-mechanically damaged }\end{array}$ \\
\hline $\begin{array}{c}\text { Synthetic } \\
\text { materials }\end{array}$ & $\begin{array}{l}\text {-Artificial collagen film (hair) } \\
\text {.Polyurethane film (skin) } \\
\text {.Human skin (putty replica) }\end{array}$ \\
\hline
\end{tabular}

Table 3.2 Hair and skin samples

Collagen film is typically used as a synthetic hair material for testing purposes.

Polyurethane films represent synthetic human skin. They are cast from human skin and have a similar surface energy, which also makes them suitable test specimens when real skin cannot be used. To characterize surface roughness of human skin, it was also replicated using a two-part silicone elastomer putty (DMR-503 Replication Putty, Dynamold, Inc., Fortworth, TX). The thickness of the film was approximately $3 \mathrm{~mm}$.

In order to simulate hair conditioner-skin contact in AFM experiments, it is important to have contact angle and surface energy of an AFM tip close to that of skin. Table 3.4 shows the contact angles and surface energies of materials important to the 
nanocharacterization of the hair samples: Untreated human hair; PDMS, which is used in conditioners; skin, which comes into contact with hair; and $\mathrm{Si}_{3} \mathrm{~N}_{4}$ film, which in the form of an AFM tip is used for nanotribological measurements [LaTorre et al., 2006]. The static contact angle of $\mathrm{Si}_{3} \mathrm{~N}_{4}$ film with high purity deionized water was measured in air by a sessile-drop method using a contact angle goniometer (Model 100, Rame-Hart Inc., Mountain Lakes, NJ, USA). $5 \mu \mathrm{L}$ of deionized water was applied on two samples using a micropipette, and three contact angle measurements were taken on each sample and averaged. 


\begin{tabular}{|c|c|c|c|c|c|c|c|c|}
\hline & Ingredient & $\begin{array}{c}\text { Damaged } \\
\text { treated } \\
\text { (commercial } \\
\text { conditioner) }\end{array}$ & $\begin{array}{l}\text { (BTMAC } \\
\text { surfactant, } \\
\text { no } \\
\text { silicone) }\end{array}$ & $\begin{array}{c}\text { (PDMS } \\
\text { blend } \\
\text { silicone, } \\
\text { low } \\
\text { deposition) } \\
\end{array}$ & $\begin{array}{l}\text { (PDMS } \\
\text { blend } \\
\text { silicone, } \\
\text { high } \\
\text { deposition) }\end{array}$ & $\begin{array}{l}\text { (Amino } \\
\text { silicone, } \\
\text { low } \\
\text { deposition) }\end{array}$ & $\begin{array}{l}\text { (Amino } \\
\text { silicone, } \\
\text { high } \\
\text { deposition) }\end{array}$ & $\begin{array}{l}\text { (BAPDMA } \\
\text { surfactant, } \\
\text { no } \\
\text { silicone) }\end{array}$ \\
\hline \multirow{3}{*}{ 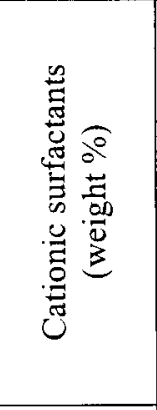 } & $\begin{array}{c}\text { Behentrimonium } \\
\text { chloride } \\
\text { (BTMAC) }\end{array}$ & - & $\mathrm{X}$ & $\mathrm{X}$ & $\mathrm{X}$ & $\mathrm{X}$ & $\mathrm{X}$ & - \\
\hline & $\begin{array}{c}\text { Behenyl } \\
\text { amidopropyl } \\
\text { dimethylamine } \\
\text { (BAPDMA) }\end{array}$ & - & - & - & - & - & - & $\mathrm{X}$ \\
\hline & $\begin{array}{c}\text { Stearamidopropyl } \\
\text { dimethylamine } \\
\text { (SAPDMA) }\end{array}$ & $\mathrm{X}$ & - & - & - & - & - & - \\
\hline 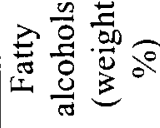 & Fatty alcohols & $\mathrm{X}$ & $\mathrm{X}$ & $\mathrm{X}$ & $\mathrm{X}$ & $\mathrm{X}$ & $\mathrm{X}$ & $\mathrm{X}$ \\
\hline \multirow{3}{*}{ 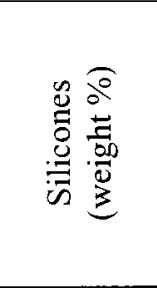 } & $\begin{array}{l}\text { PDMS blend } \\
\text { (Dimethicone) }\end{array}$ & $\mathrm{X}$ & - & - & - & - & - & - \\
\hline & $\begin{array}{l}\text { PDMS blend } \\
\text { (with blend of } \\
\text { high MW) }\end{array}$ & - & - & Low & High & - & - & - \\
\hline & $\begin{array}{l}\text { Amino } \\
\text { silicone }\end{array}$ & - & - & - & - & Low & High & - \\
\hline \multicolumn{9}{|c|}{ Deposition levels for conditioner ingredients on hair } \\
\hline \multicolumn{2}{|c|}{$\begin{array}{l}\text { Cationic surfactant } \\
\text { deposition level }\end{array}$} & $\begin{array}{l}\text { Similar to } \\
\text { commercial } \\
\text { conditioner }\end{array}$ & $\mathrm{X}$ & $\mathrm{X}$ & $\mathrm{X}$ & $\mathrm{X}$ & $\mathrm{X}$ & $\mathrm{X}$ \\
\hline \multicolumn{2}{|c|}{ Fatty alcohol deposition level } & $\begin{array}{l}\text { Similar to } \\
\text { commercial } \\
\text { conditioner }\end{array}$ & $\mathrm{X}$ & $\mathrm{X}$ & $\mathrm{X}$ & $\mathrm{X}$ & $\mathrm{X}$ & $\mathrm{X}$ \\
\hline \multicolumn{2}{|c|}{ Silicone deposition level } & $\begin{array}{l}\text { Similar to } \\
\text { commercial } \\
\text { conditioner }\end{array}$ & None & Low & High & Low & High & None \\
\hline
\end{tabular}

Table 3.3 Matrix of hair samples treated with various combinations of ingredients 


\begin{tabular}{|c|c|c|}
\hline & Contact angle $\left({ }^{\circ}\right)$ & Surface energy $(\mathrm{N} / \mathrm{m})$ \\
\hline 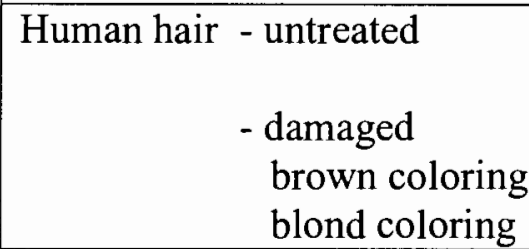 & $\begin{array}{l}103^{\mathrm{a}} \\
100^{\mathrm{b}} \\
60^{\mathrm{b}} \\
55^{\mathrm{b}}\end{array}$ & $\begin{array}{l}0.024^{\mathrm{a}} \\
0.028^{\mathrm{b}} \\
0.038^{\mathrm{b}} \\
0.047^{\mathrm{b}}\end{array}$ \\
\hline PDMS (bulk) & $105^{\mathrm{c}}$ & $0.020^{\mathrm{d}}$ \\
\hline $\begin{array}{c}\text { Human skin } \text { - forehead } \\
\text { - forearm } \\
\text { - finger }\end{array}$ & $\begin{array}{l}55^{\mathrm{e}} \\
88^{\mathrm{e}} \\
84^{\mathrm{f}} \\
88^{\mathrm{f}} \\
104^{\mathrm{g}} \text { (soap-washed) } \\
58^{\mathrm{g}} \text { (no pretreatment) } \\
\end{array}$ & $\begin{array}{l}0.043^{\mathrm{e}} \\
0.038^{\mathrm{e}} \\
0.029^{\mathrm{f}} \\
0.024^{\mathrm{f}} \\
0.027^{\mathrm{g}}\end{array}$ \\
\hline $\mathrm{Si}_{3} \mathrm{~N}_{4}$ film & $35^{\mathrm{h}}$ & $0.047^{1}$ \\
\hline $\begin{array}{l}\text { Molina et al. [2001] } \\
\text { aTorre et al. [2006] } \\
\text { 3hushan and Burton [2005] } \\
\text { albert et al. [1993] } \\
\text { erebour et al. [2000] } \\
\text { chott [1971] } \\
\text { Ginn et al. [1968] } \\
\text {-aTorre et al. [2006] } \\
\text { lanazawa [1984] }\end{array}$ & & \\
\hline
\end{tabular}

Table 3.4 Contact angle and surface energy of relevant materials associated with nanotribological characterization of hair. 


\section{CHAPTER 4}

\section{RESULTS AND DISCUSSION}

\subsection{Surface roughness, friction, and adhesion for various ethnicities of hair}

Topographical images of Caucasian, Asian, and African hair were taken up to scan sizes of $20 \times 20 \mu \mathrm{m}^{2}$, as shown in Figure 4.1. Lighter areas of the images correspond to higher topography, and darker areas correspond to lower topography. Only virgin and chemo-mechanically damaged hair are shown in Figure 4.1 because virgin treated samples closely resemble virgin hair samples. One can see the variation in cuticle structure even in virgin hair. Cracking and miscellaneous damage at the cuticle edges is evident at both virgin and chemo-mechanically damaged conditions. In virgin hair, the damage is likely to be caused by mechanical damage resulting from daily activities such as washing, drying, and combing. Most of the virgin cuticle scales that were observed, however, were relatively intact. Long striations similar to scratches, and "scale edge ghosts" (outlines of a former overlying cuticle scale edge left on the underlying scale before it was broken away) were found on the surface. In some instances, the areas surrounding the cuticle edges appeared to show residue or debris on the surface, which is most likely remnants of a previous cuticle or the underside of the cuticle edges that are now exposed (such as the endocuticle). Caucasian and Asian virgin hair displayed similar surface structure, while the African hair samples showed more signs of endocuticular 
Surface roughness maps of various hair

Caucasian hai
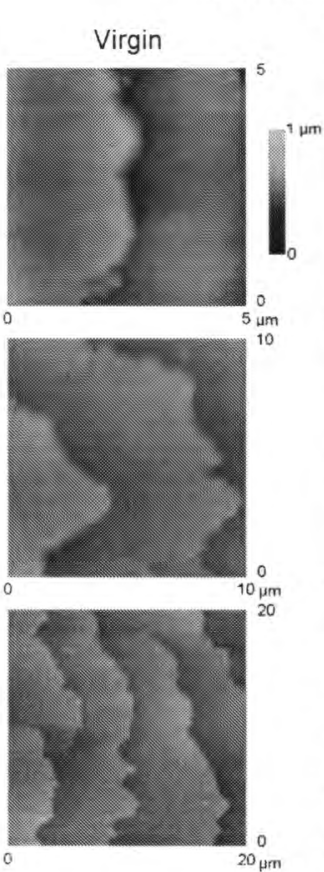

Chemo-mechanically damaged

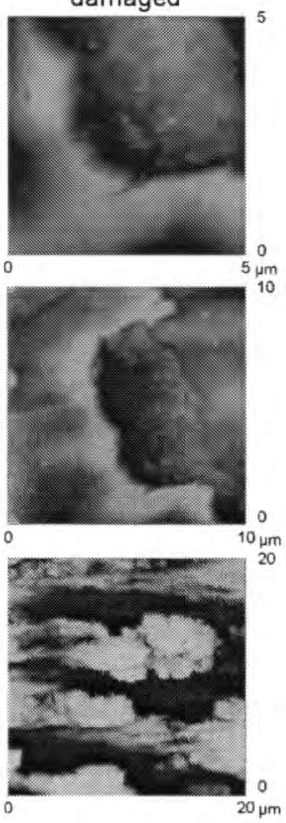

African hair
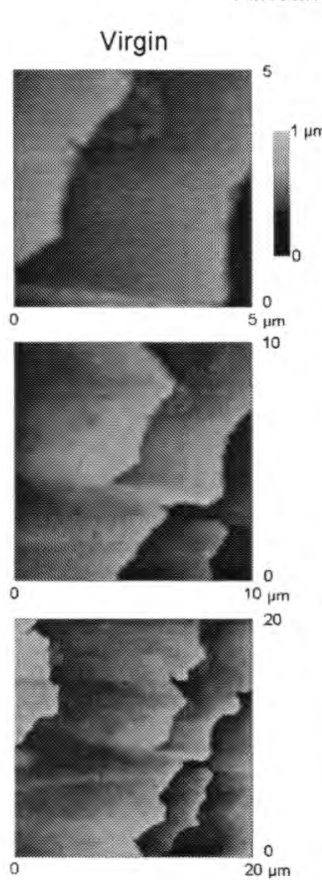

Asian hair

Chemo-mechanically

Virgin
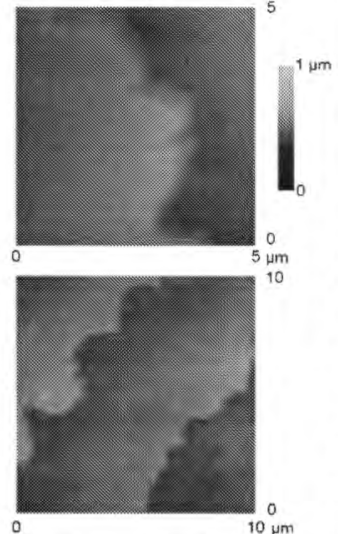

$10+\mathrm{m}$

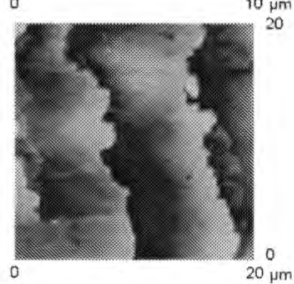

Chemo-mechanically

damaged
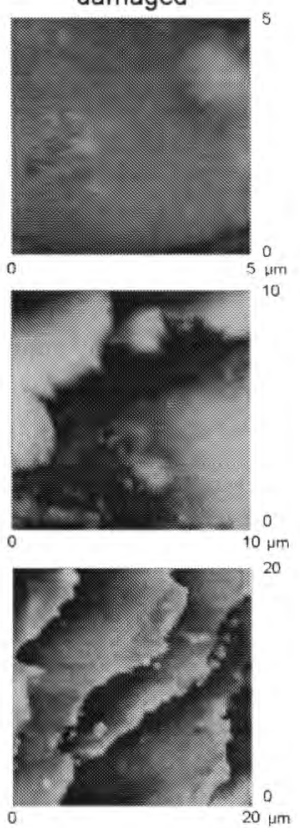

Fig. 4.1 Surface roughness of virgin and chemo-mechanically damaged Caucasian, Asian, and African hair at 5, 10, and $20 \mu \mathrm{m}^{2}$ scan sizes [LaTorre and Bhushan, 2005a]. 
remains along the scale edges. One can also see more curvature in the cuticle scales of African hair, which is attributed to its elliptical cross-sectional shape and curliness, which can partially uplift the scales in different places. With respect to chemo-mechanically damaged hair, it is observed that several regions seem to exist in these hair samples, ranging from intact cuticle scales to high levels of wear on the surface. In many cases these regions occur side by side. This wide variation in chemo-mechanically damaged cuticle structure results in a wider range of tribological properties on the micro/nanoscale for these fibers. Caucasian and Asian chemo-mechanically damaged hair showed more worn away cuticle scales than in chemo-mechanically damaged African hair, which showed mostly endocuticle remnants. This is most likely due to the different effects that chemical straightening has on the hair versus multiple cycles of perming the hair.

A more focused look into roughness and friction on the cuticle surface can be found by comparing Caucasian, Asian, and African virgin and chemo-mechanically damaged hair, Figure 4.2(a) and (b), and virgin and virgin treated hair, Figure 4.3(a) and (b). Virgin hair was used as the baseline to compare variations in roughness and friction force against modified hair (chemo-mechanically damaged or virgin treated). Scan sizes of $5 \times 5 \mu \mathrm{m}^{2}$ and $10 \times 10 \mu \mathrm{m}^{2}$ are displayed. Above each AFM and FFM image are crosssectional plots of the surface (taken at the accompanying arrows) corresponding to surface roughness or friction force, respectively. From the surface roughness images, the step heights of one or more cuticle edges can be clearly seen. Step heights range from approximately 0.3 to $0.5 \mu \mathrm{m}$.

If the surface is assumed to have Gaussian height distribution and an exponential autocorrelation function, then the surface can be statistically characterized by just two 
Surface roughness and friction force maps of virgin and chemo-mechanically damaged hair
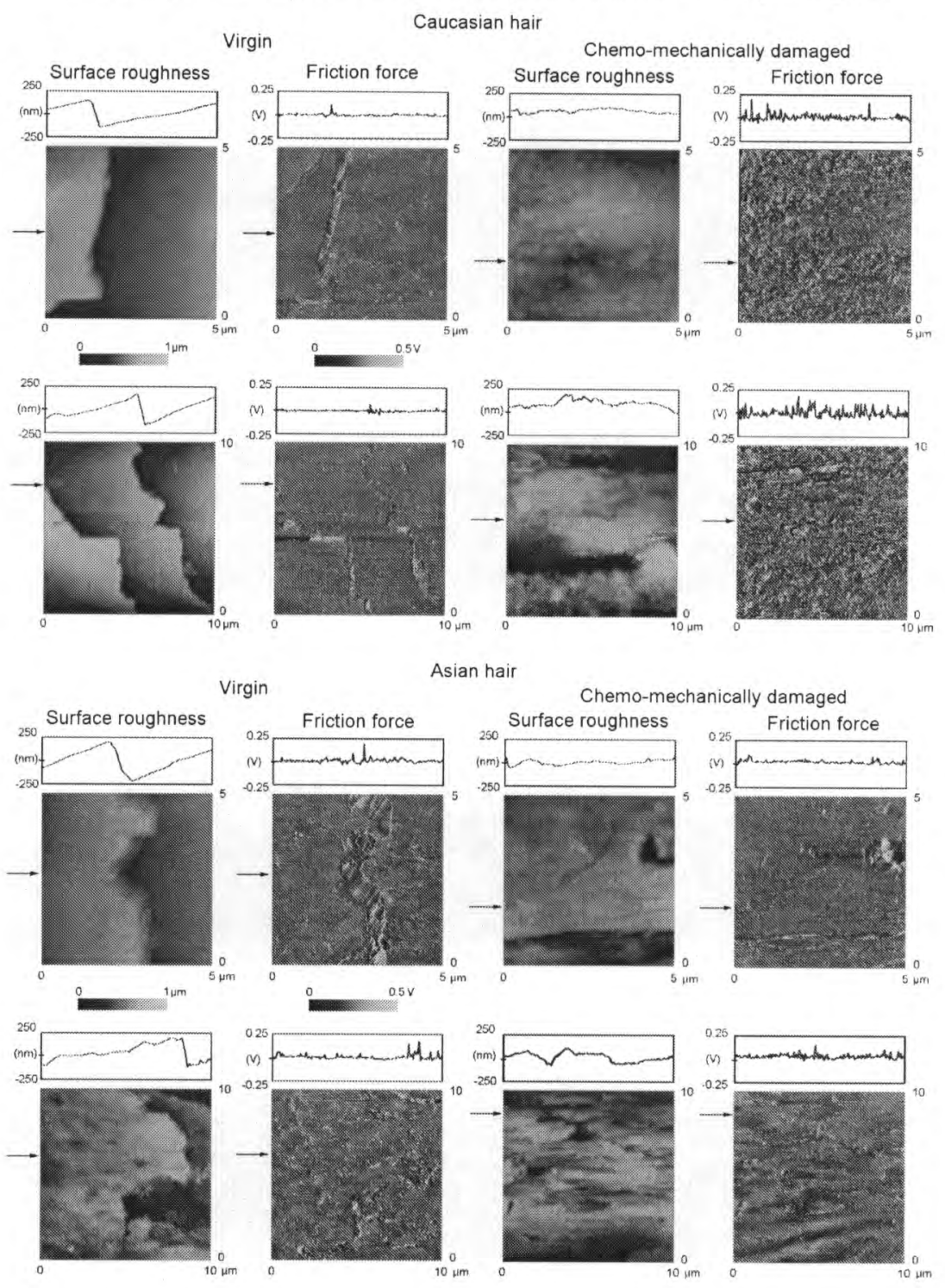

(a)

Continued

Fig. 4.2 (a) Surface roughness and friction images for virgin and chemo-mechanically damaged Caucasian, Asian hair at 5 and $10 \mu \mathrm{m}^{2}$ scan sizes. Shown above each image is a cross section taken at the corresponding arrows to show roughness and friction force information [LaTorre and Bhushan, 2005a] (b) Surface roughness and friction force images for virgin and chemo-mechanically damaged African hair at 5 and $10 \mu \mathrm{m}^{2}$ scan sizes. 
Fig. 4.2 continued

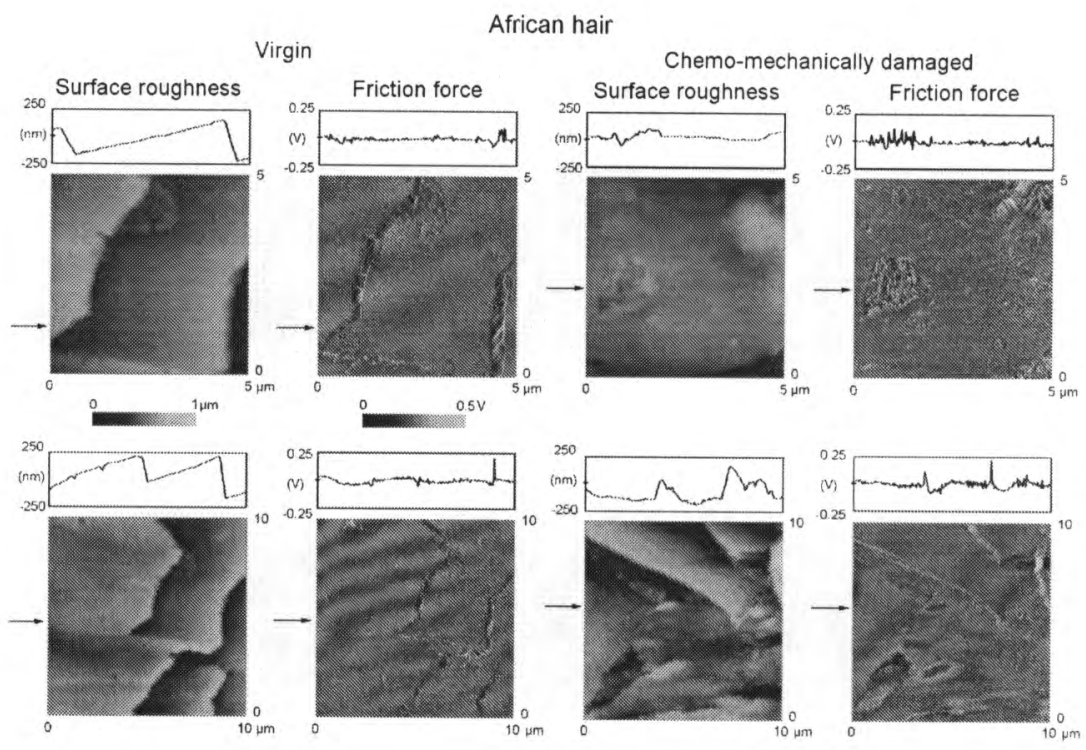

(b) 


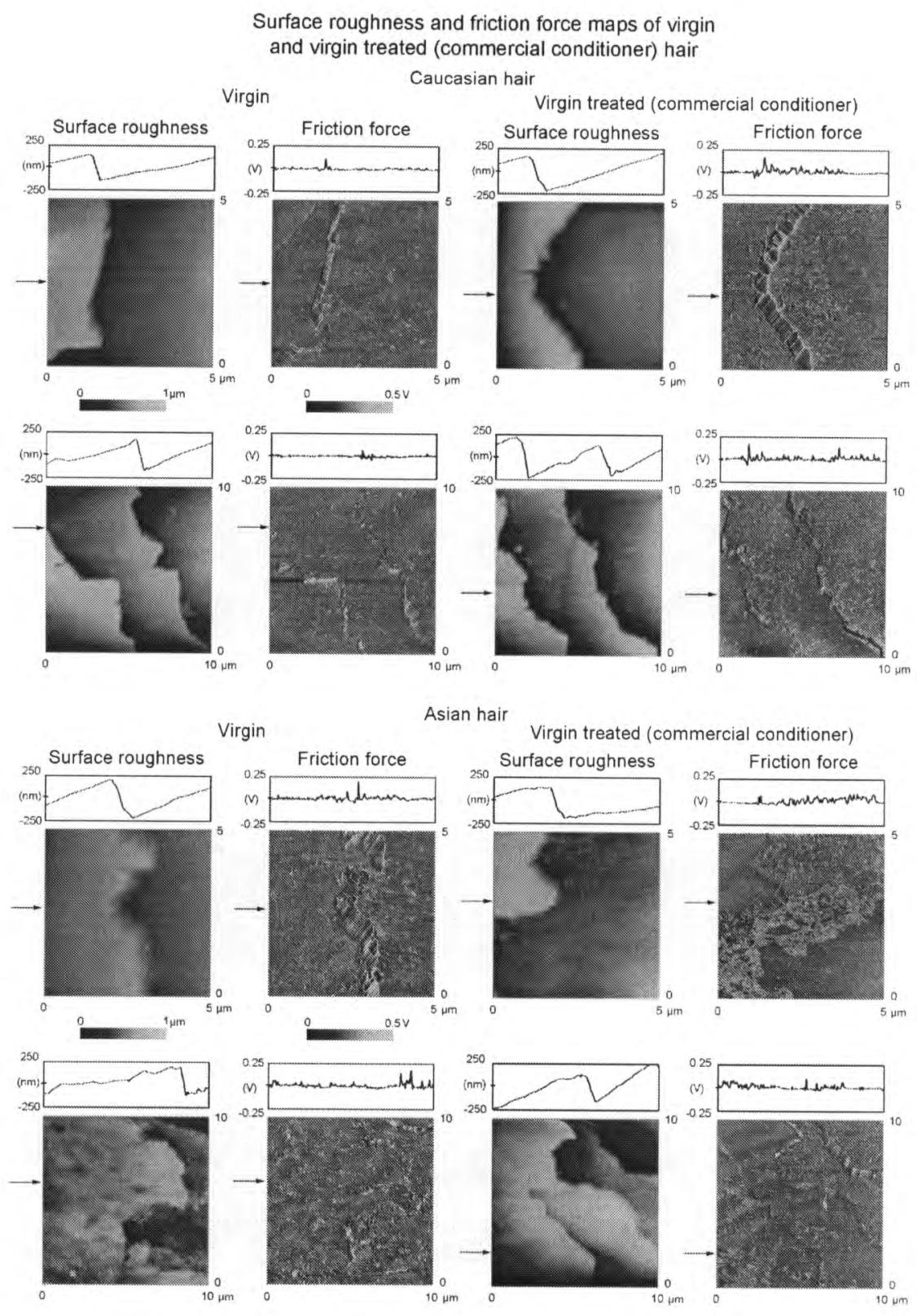

(a)

Continued

Fig. 4.3 (a) Surface roughness and friction images for virgin and virgin treated Caucasian, Asian hair at 5 and $10 \mu \mathrm{m}^{2}$ scan sizes. Shown above each image is a cross section taken at the corresponding arrows to show roughness and friction force information [LaTorre and Bhushan, 2005a] (b) Surface roughness and friction force images for virgin and virgin treated African hair at 5 and $10 \mu \mathrm{m}^{2}$ scan sizes. 
Fig 4.3 continued

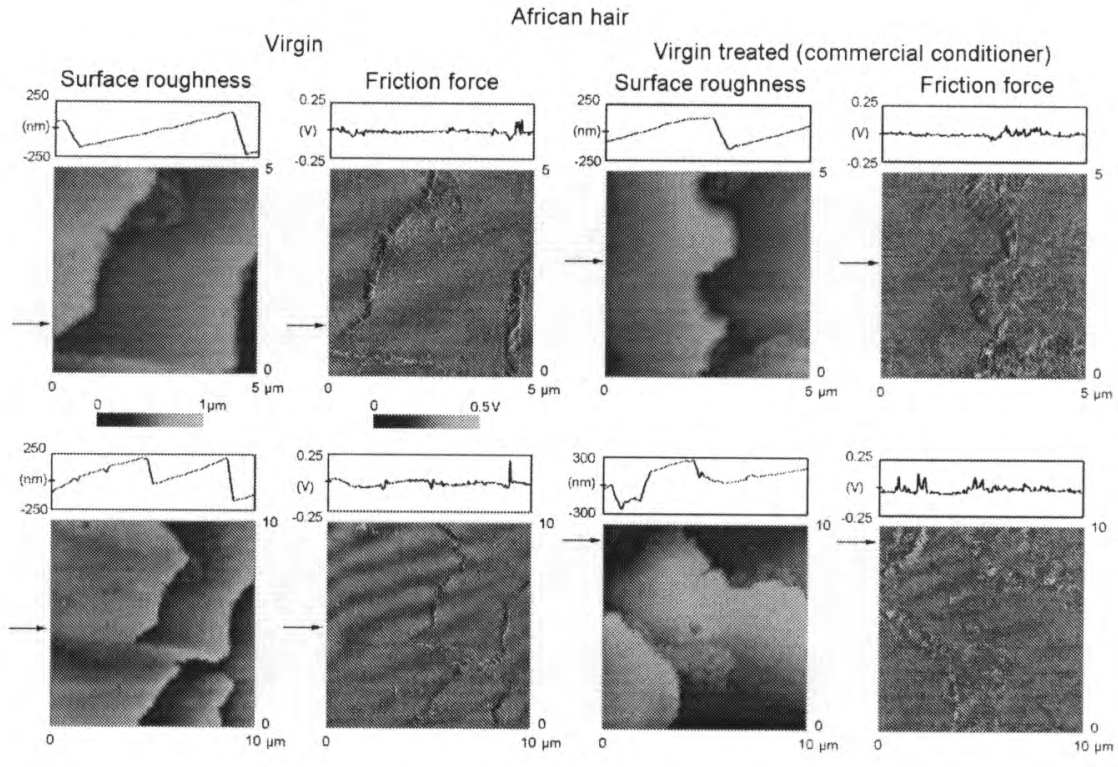

(b) 
parameters: a vertical descriptor, height standard deviation $\sigma$, and a spatial descriptor, correlation distance $\beta^{*}$ [Bhushan, 1999a, b, 2002]. The standard deviation $\sigma$ is the square root of the arithmetic mean of the square of the vertical deviation from the mean line. The correlation length can be referred to as the length at which two data points on a surface profile can be regarded as being independent, thus serving as a randomness measure [Bhushan, 2002].

Table 4.1 displays these roughness parameters for each ethnicity as a function of hair type (virgin, chemo-mechanically damaged, and virgin treated) [LaTorre and Bhushan, 2005a]. Virgin hair was shown to generally have the lowest roughness values, with virgin treated hair closely resembling virgin hair. Chemo-mechanically damaged hair showed a significantly higher standard deviation of surface height. This variation is expected because of the non-uniformity of the mechanical and chemical damage that occurs throughout a whole head of hair as well as each individual fiber. This is in agreement with the images of chemo-mechanically damaged hair shown previously, where regions of intact cuticle and severe degradation of the surface are intermingled. The trends observed for standard deviation were not as evident for the correlation length $\beta^{*}$. For each ethnicity, chemo-mechanically damaged and virgin treated hair showed similar $\beta^{*}$ values.

From Figure 4.2(a) and (b), friction forces are generally seen to be higher on chemo-mechanically damaged hair than on virgin hair. Although friction forces were similar in magnitude, it was observed that the friction force on the cuticle surface of chemo-mechanically damaged hair showed a much larger variance, which contributed to 
the higher friction values. Another contribution to the higher friction could be that the tiny peaks developed after damage also create a ratchet mechanism on a nanoscale, which

\begin{tabular}{|c|c|c|c|c|c|c|}
\hline & \multicolumn{2}{|c|}{ Virgin hair } & \multicolumn{2}{|c|}{$\begin{array}{l}\text { Chemo- } \\
\text { mechanically } \\
\text { damaged hair }\end{array}$} & \multicolumn{2}{|c|}{$\begin{array}{l}\text { Virgin treated hair } \\
\text { (commercial } \\
\text { conditioner) }\end{array}$} \\
\hline \multicolumn{7}{|c|}{ Surface roughness parameters $\left(\sigma(\mathrm{nm}), \beta^{*}(\mu \mathrm{m})\right)$} \\
\hline & $\sigma(\mathrm{nm})$ & $\beta^{*}(\mu \mathrm{m})$ & $\sigma(\mathrm{nm})$ & $\beta^{*}(\mu \mathrm{m})$ & $\sigma(\mathrm{nm})$ & $\beta^{*}(\mu \mathrm{m})$ \\
\hline Caucasian & $12 \pm 8$ & $\begin{array}{c}0.61 \\
\pm 0.3 \\
\end{array}$ & $17 \pm 10$ & $1.0 \pm 0.3$ & $12 \pm 4$ & $\begin{array}{c}0.90 \\
\pm 0.3 \\
\end{array}$ \\
\hline Asian & $9.7 \pm 4$ & $\begin{array}{c}0.73 \\
\pm 0.3 \\
\end{array}$ & $33 \pm 15$ & $\begin{array}{r}0.94 \\
\pm 0.3 \\
\end{array}$ & $\begin{array}{c}7.1 \\
\pm 0.1 \\
\end{array}$ & $\begin{array}{c}0.97 \\
\pm 0.3 \\
\end{array}$ \\
\hline African & $12 \pm 5$ & $\begin{array}{c}0.92 \\
\pm 0.3 \\
\end{array}$ & $21 \pm 16$ & $\begin{array}{r}0.78 \\
\pm 0.3 \\
\end{array}$ & $11 \pm 4$ & $\begin{array}{r}0.89 \\
\pm 0.2 \\
\end{array}$ \\
\hline \multicolumn{7}{|c|}{ Average coefficient of friction $\mu$} \\
\hline Caucasian & 0.02 & 0.01 & 0.13 & $=0.05$ & 0.03 & 0.01 \\
\hline Asian & 0.03 & 0.01 & 0.13 & $=0.04$ & 0.06 & 0.04 \\
\hline African & 0.04 & 0.02 & 0.14 & $=0.08$ & 0.05 & 0.01 \\
\hline \multicolumn{7}{|c|}{ Adhesive force $F_{m}(\mathrm{nN})$} \\
\hline Caucasian & \multicolumn{2}{|c|}{25} & \multicolumn{2}{|c|}{16} & \multicolumn{2}{|c|}{32} \\
\hline Asian & \multirow{2}{*}{\multicolumn{2}{|c|}{$\frac{31}{35}$}} & \multicolumn{2}{|c|}{18} & \multicolumn{2}{|c|}{79} \\
\hline African & & & \multicolumn{2}{|c|}{38} & \multicolumn{2}{|c|}{63} \\
\hline
\end{tabular}

Table 4.1 Surface roughness, coefficient of friction, and adhesive force values of virgin, chemo-mechanically damaged, and virgin treated (1 cycle commercial conditioner) hair at each ethnicity.

affects the friction between the AFM tip and the surface. These peaks could then add to the friction signal. The damage of the hair by chemical and mechanical means have shown high reproducibility in the lab in terms of structure alteration, which explains the 
similar friction properties no matter the ethnicity for chemo-mechanically damaged hair. With virgin and virgin treated hair, however, it is unknown what prior mechanical damage and sun exposure the fibers have seen, and varies largely on the individuals. Thus, across ethnicity there is variability in friction force for those hair.

Perhaps the most notable difference between virgin and virgin treated hair fibers can be seen in the friction force mappings of Figure 4.3(a) and (b). Although quite comparable in surface roughness, close examination of the virgin treated hair surface shows an increase in friction force, usually only surrounding the bottom edge of the cuticle. This was unlike virgin hair, where friction generally remained constant along the surface, and unlike chemo-mechanically damaged hair, where there was large variability which was random over the entire surface.

Figure 4.4 presents friction force curves as a function of normal load for Caucasian virgin, chemo-mechanically damaged, and virgin treated hair to further illustrate the previous discussion. One can see a relatively linear relationship between the data points for each type of hair sample. When plotted in such a way, the coefficient of friction is determined by the slope of the least squares fit line through the data. If this line is extended to intercept the horizontal axis, then a value for adhesive force can also be calculated, since friction force $F$ is governed by the relationship

$$
F=\mu\left(W+F_{a}\right)
$$

where $\mu$ is the coefficient of friction, $W$ is the applied normal load, and $F_{a}$ is the adhesive force [Bhushan, 1999a, 2002].

One explanation for the increase in friction force of virgin treated hair on the micro/nanoscale is that during tip contact meniscus forces between the tip and the 
conditioner/cuticle become large as the tip rasters over the surface, causing an increase in the adhesive force. This adhesive force is of the same magnitude as the normal load, which makes the adhesive force contribution to friction rather significant. Thus, at sites where conditioner is accumulated on the surface (namely around the cuticle scale edges), friction force actually increases. On the macroscale, however, the adhesive force is much lower in magnitude than the applied normal load, so the adhesive force contribution to friction is negligible over the hair switch. As a result, virgin treated hair shows a decrease in friction force on the macroscale, which is opposite the micro/nanoscale trend. The friction and adhesion data on the micro/nanoscale is useful, though, because it relates to the presence of conditioner on the cuticle surface and allows for obtaining an estimate of conditioner distribution.

It is also observed from Figure 4.4 that while chemo-mechanically damaged hair displays a higher friction force on the application of normal load, and consequently a higher coefficient of friction, chemo-mechanically damaged hair friction is not as strongly dependant on adhesive force contribution as the virgin and virgin treated hair. Average values for $\mu$ were calculated and are compiled in Figure 4.5, and Table 4.1 for all hair ethnicities and types [LaTorre and Bhushan, 2005a]. Error bars represent $\pm 1 \sigma$ on the average coefficient value. The coefficients of friction for virgin, chemo-mechanically damaged, and virgin treated Caucasian hair are 0.02, 0.13, and 0.03, respectively. For virgin, chemo-mechanically damaged, and virgin treated Asian hair, coefficients of friction are $0.03,0.13$, and 0.06 , respectively. Finally, virgin, chemomechanically damaged, and virgin treated African hair coefficients of friction are 0.04 , 
0.14 , and 0.05 , respectively. Chemo-mechanically damaged hair presents the highest coefficient of friction, but also displays the largest standard deviation, due to the large

Friction force vs. normal load curves for

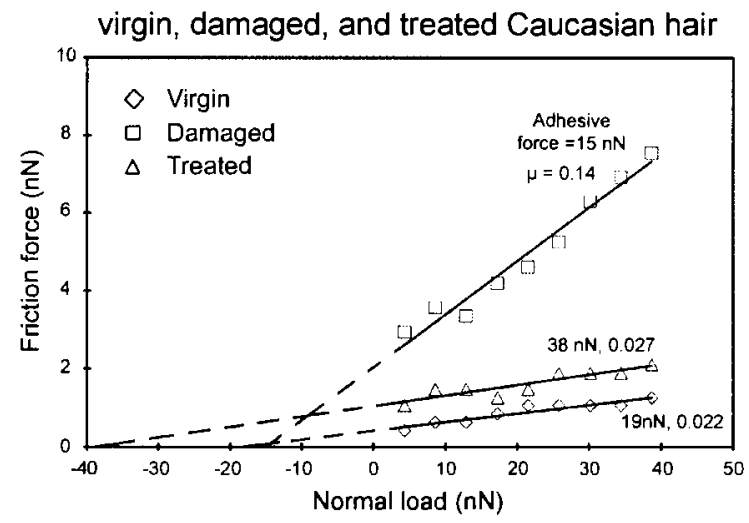

Coefficient of friction comparison for virgin, chemomechanically damaged, and virgin treated (commercial conditioner) hair

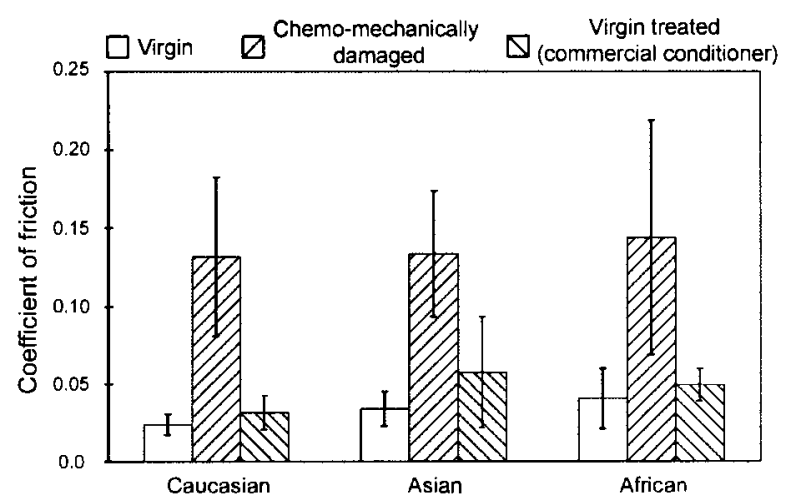

Fig. 4.4 Friction force vs. normal load curves showing typical values for virgin, chemomechanically damaged, and virgin treated Caucasian hair. Error bars represent $\pm 1 \sigma$ on the average coefficient value. In the bottom plot, average coefficient of friction values for virgin, damaged, and treated hair at each ethnicity [LaTorre and Bhushan, 2005a]. 
variations in chemical and mechanical damage that each hair or hair bundle experiences. Coefficient of friction of virgin treated hair is slightly larger than that of virgin hair for all ethnicities. While the coefficient of friction is similar in virgin and virgin treated hair, the adhesive force contribution to friction for Caucasian virgin treated hair is higher than in Caucasian virgin hair, when calculated according to the method described above. However, this was not always the trend for Asian and African virgin treated hair samples. It should be noted that since in friction force measurement the tip moves laterally over the surface, this might cause a smearing out of the conditioner layer which accounts for the inconsistent trend. In the adhesive force mappings described in the next section, where determination of adhesive force does not depend on this lateral movement, all virgin treated hair samples showed higher adhesion than their virgin hair counterparts.

A force calibration plot (FCP) technique and resulting adhesive force maps (commonly called force-volume maps) can be used to understand the adhesive forces between the AFM tip and the sample [Bhushan, 1999b, 2005; Bhushan and Dandavate, 2000; Liu and Bhushan, 2003]. Shown in Figure 4.6 are FV maps and an example of the individual force calibration plots from which the maps were created. Adhesive force distribution for chemo-mechanically damaged hair was shown to be comparable to virgin hair adhesive force values, but slightly lower. A significant increase in adhesive force over the entire mapping was found in all cases of virgin treated hair as compared to virgin hair, especially in Asian and African hair. Conditioner distribution can be seen from these images. This technique shows promise to be very useful in further study of the distribution of materials and hair care products on the surface of the hair. 
A typical value for the adhesive force of each FV map was calculated. Values are shown in the plot of Figure 4.5, along with surface roughness and coefficient of friction data for all hair samples. Adhesive force values are also tabulated in Table 4.1. 
Surface roughness, coefficient of friction, and adhesive force data for various hair

\section{Caucasian}

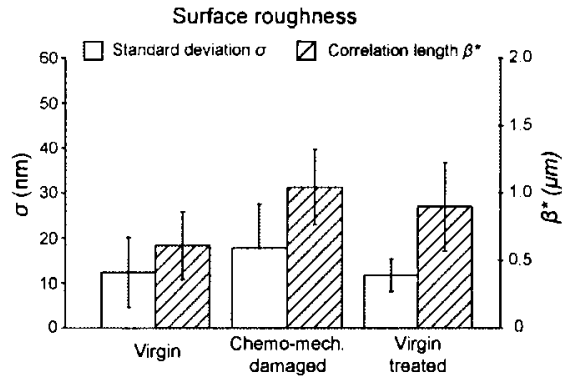

Surface roughness

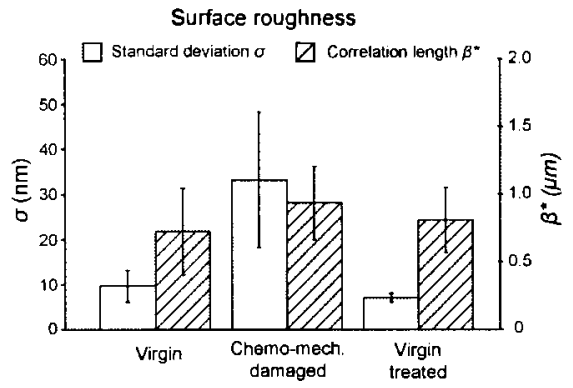

Surface roughness

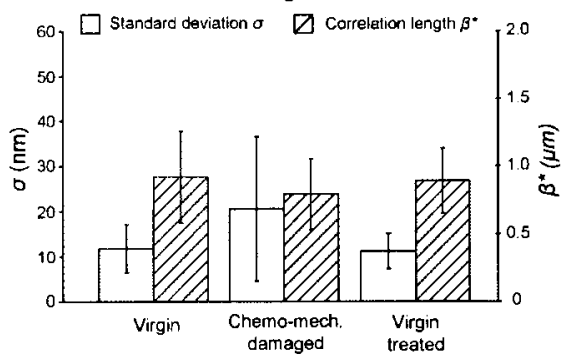

Coefficient of friction

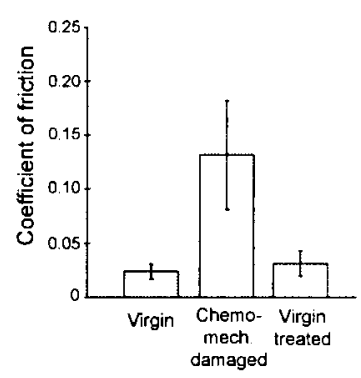

Asian

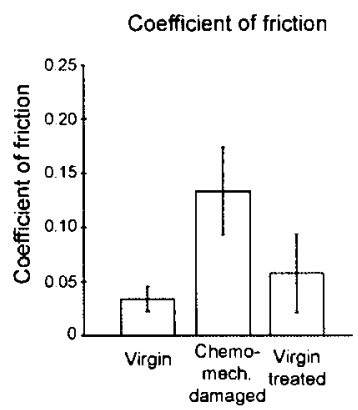

African

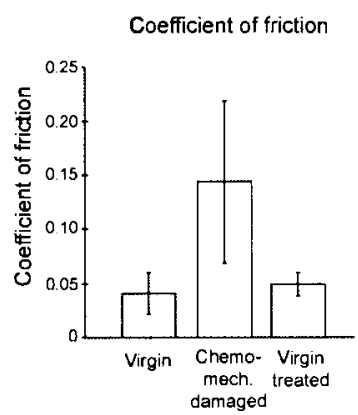

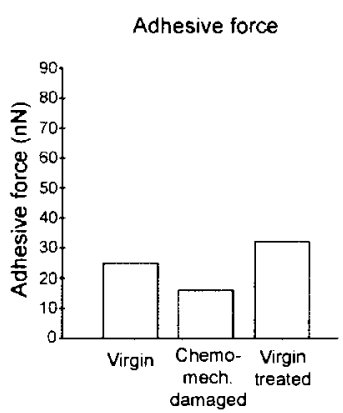

Adhesive force

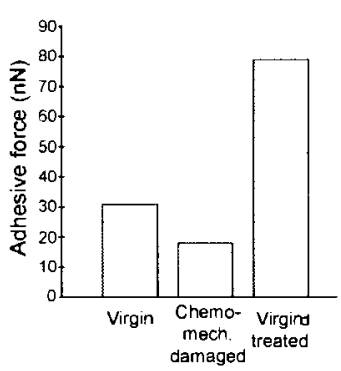

Adhesive force

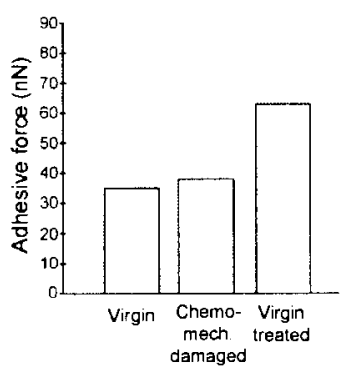

Fig. 4.5 Surface roughness, coefficient of friction, and adhesive force data for virgin, chemo-mechanically damaged, and virgin treated hair at each ethnicity [LaTorre and Bhushan, 2005a]. 

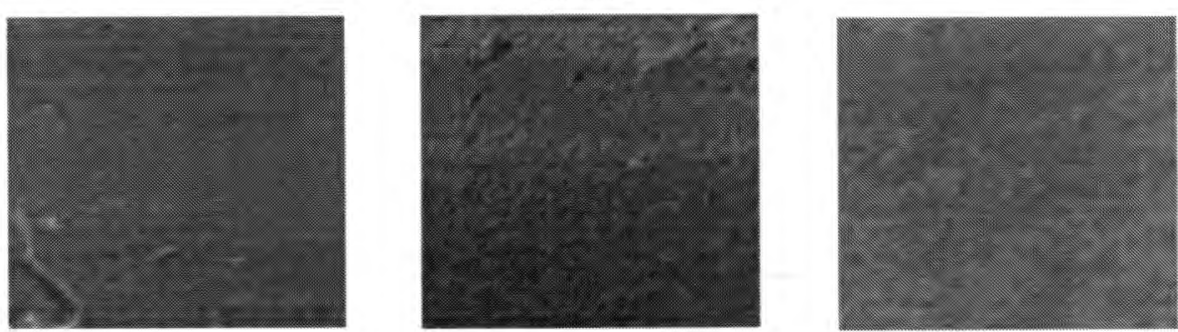

Asian
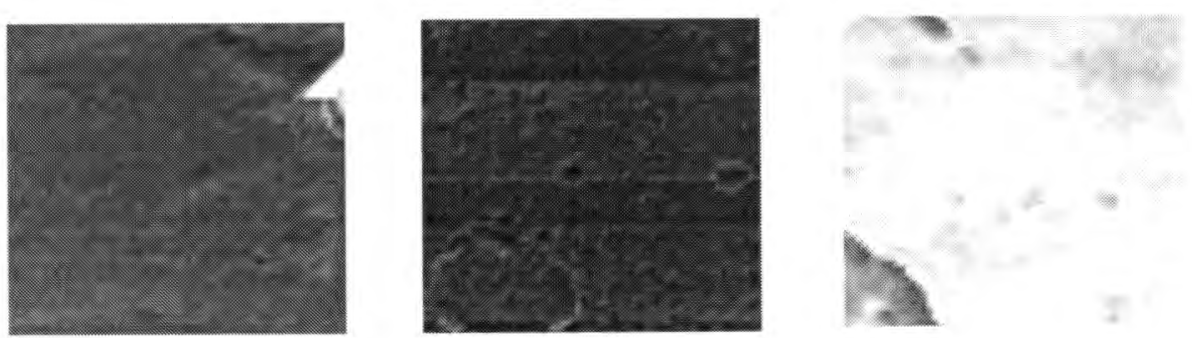

African
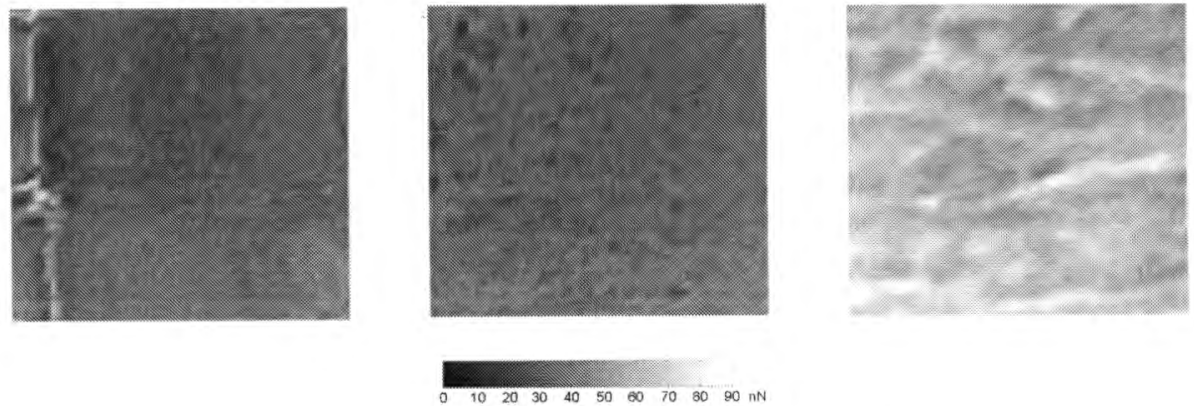

Individual force calibration plots for Caucasian virgin, chemo-mechanically damaged, and virgin treated (commercial conditioner) hair
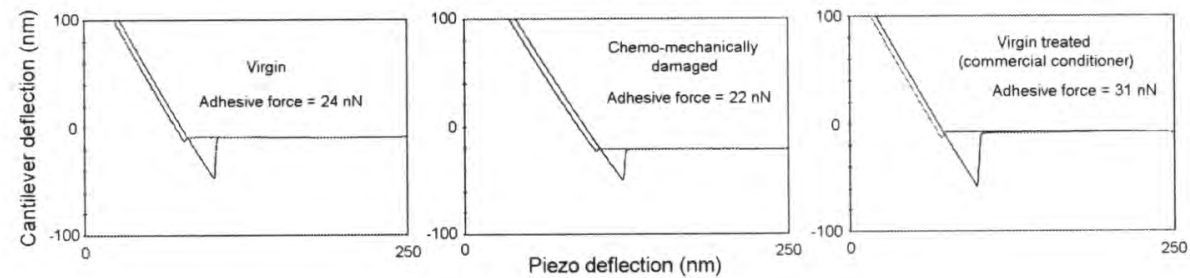

Fig. 4.6 Force volume maps of virgin, chemo-mechanically damaged, and virgin treated hair at each ethnicity. Examples of the individual force calibration plots, which make up the FV maps, are presented for Caucasian hair of each type [LaTorre and Bhushan, 2005a]. 


\subsubsection{Directionality effects of friction on the nanoscale}

The outer surface of human hair is composed of numerous cuticle scales running along the fiber axis, generally stacked on top of each other. As previously discussed, the heights of these step changes are approximately $300 \mathrm{~nm}$. These large changes in topography make the cuticle an ideal surface for investigating the directionality effects of friction using AFM/FFM.

The directionality effect of friction on the macroscale has been well studied in the past. It was shown by Robbins [1994] and Bhushan et al. [2005] that rubbing the hair from the tip to the root (against the cuticle steps) results in a higher coefficient of friction than rubbing the hair from root to tip (with the cuticles). On the micro/nanoscale, it is important to distinguish how material effects and topography-induced effects contribute to the directionality effect of friction force when scanning over and back across a small surface region [Bhushan, 1999b, 2002, 2005]. Figure 4.7 shows surface roughness, friction force, and surface slope maps of a Caucasian virgin hair fiber, each coupled to their accompanying $2 \mathrm{D}$ cross-sectional profiles. The scan size of $5 \times 5 \mu \mathrm{m}^{2}$ provides one cuticle step height to be studied. As the tip rasters over the step in the trace mode (i.e. from left to right), a small decrease in the friction force is observed as the tip follows the step downward. When the tip comes back in the retrace mode (i.e. right to left), climbing up the sharp peak results in a high friction signal. However, because of the sign convention of the AFM/FFM that causes a reversal in the sign when traversing the opposite direction, this signal is now observed to be highly negative. The interesting difference between the two profiles lies in the fact that the magnitude of the decrease in friction when going up the step is much larger than the magnitude of the friction when the 
Directionality effects of friction
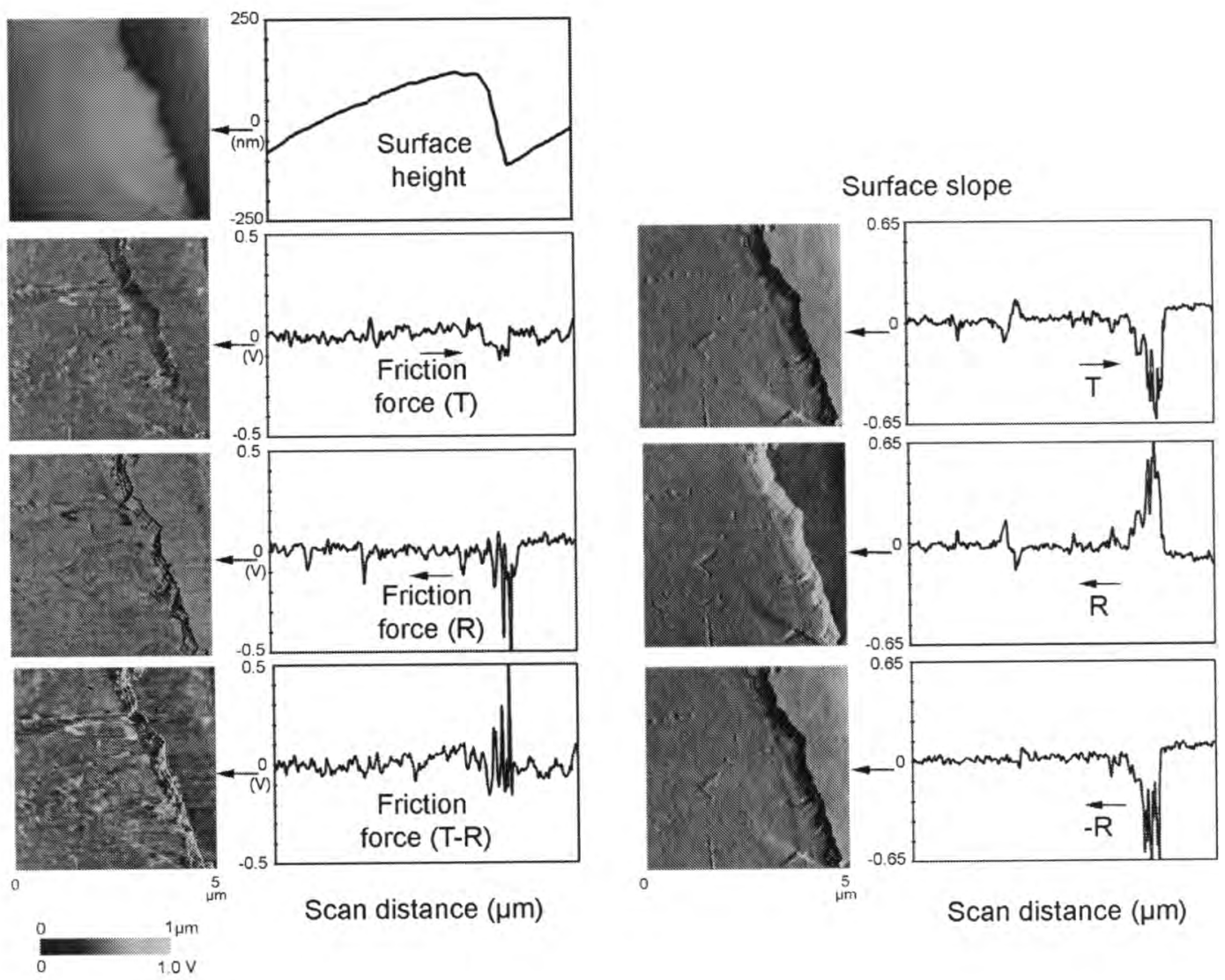

Fig. 4.7 Surface roughness, friction force, and slope across a cuticle scale edge to show the directionality dependence of friction. Left: Surface roughness and friction force mappings with accompanying 2D profiles. Right: Surface slope mappings with accompanying 2D profiles [LaTorre and Bhushan, 2005a]. 
tip is going down the step, yet both signals are in the same direction. The important result is that even by subtracting the two signals (T-R), there is still a gross variation in the image due to topography effects. These topography effects yield friction variations in the same direction, whereas material effects show up in opposite directions. It can be shown that the cuticle edge provides a local ratchet and collision mechanism that increases the friction signal at that point. It was concluded that surface slope variation always correlates to friction force variations with respect to topography effects, and the data presented in Figure 4.7 shows the same trend when comparing trace and negative retrace slope profiles.

\subsection{Surface roughness, friction, and adhesion for virgin and chemically damaged Caucasian hair (with and without commercial conditioner treatment)}

The hair surface is negatively charged and can be damaged by a variety of chemical (permanent hair waving, chemical relaxation, coloring, bleaching) and mechanical (combing, blowdrying) factors [Robbins, 1994; Bolduc and Shapiro, 2001; Gray, 2001]. Figure 4.8 shows the transformation and wear of the cuticles scales before and after damage. Chemical damage causes parts of the scales to fracture and reveal underlying cuticle remnants. Conditioner application provides a protective coating to the hair surface for prevention of future damage.

Shown in Figure 4.9 are surface roughness and friction force plots for virgin, virgin treated, chemically damaged, chemically damaged treated ( 1 cycle conditioner), and chemically damaged treated ( 3 cycles conditioner). Above each AFM and FFM image are cross-sectional plots of the surface (taken at the accompanying arrows) corresponding to surface roughness and friction force, respectively. Although virgin and 
Virgin hair

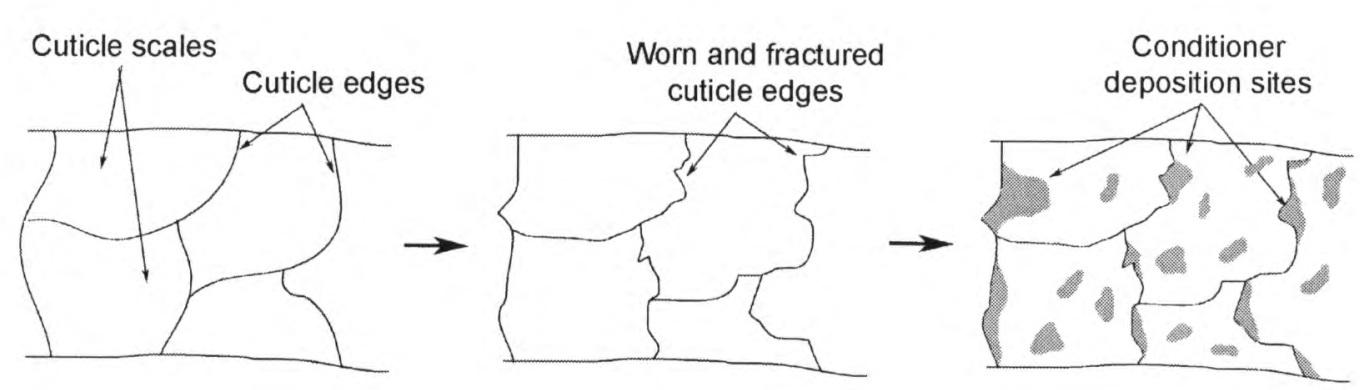

Damaged hair

Worn and fractured cuticle edges
Damaged hair treated with conditioner

Untreated and treated hair fiber cross-section

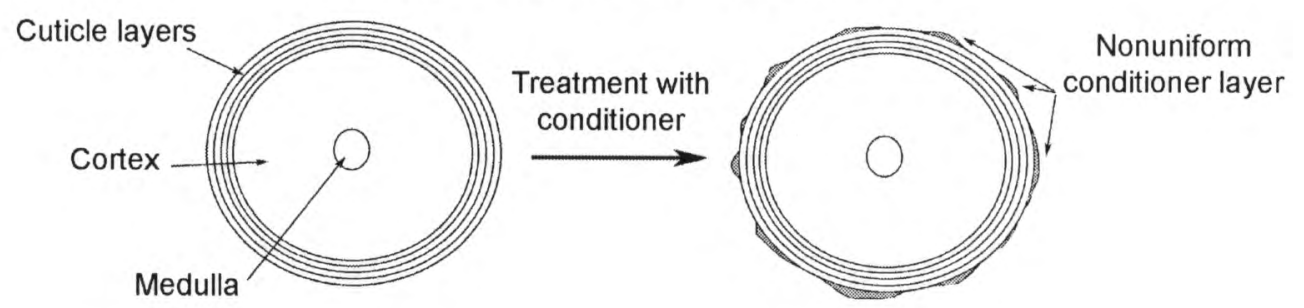

Fig. 4.8 The effect of damage to the cuticle scales and the deposition of conditioner on the cuticle surface. The cross-section of the hair with and without conditioner is shown below [LaTorre et al., 2006]. 

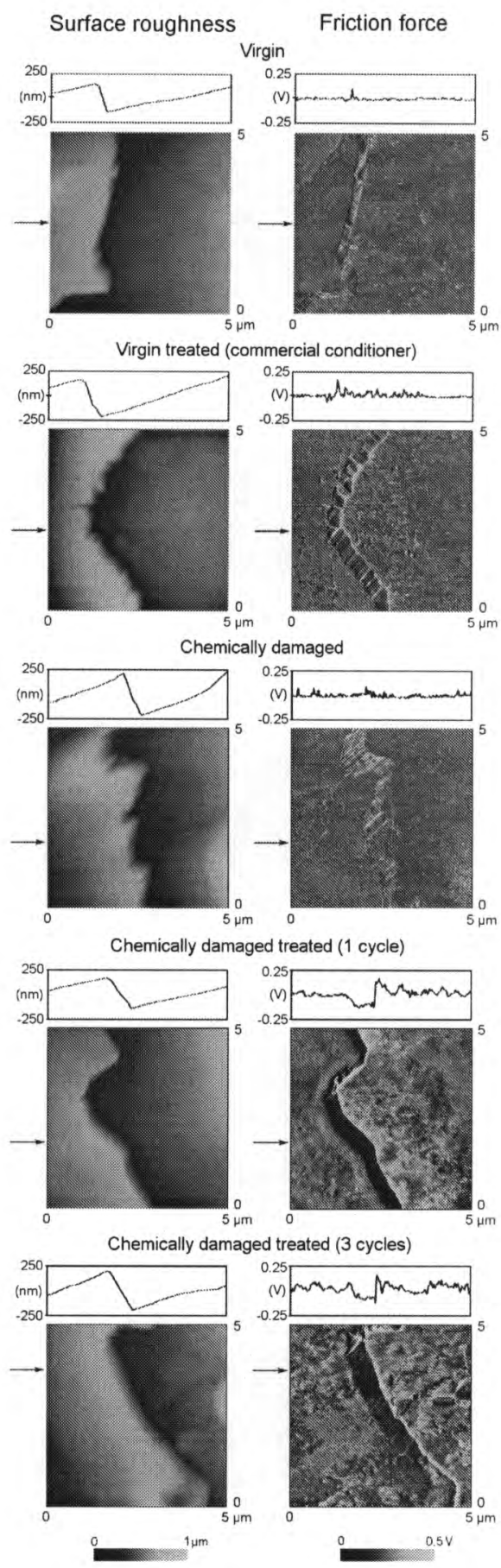

Fig. 4.9 Surface roughness and friction force images for virgin, virgin treated, damaged, damaged treated ( 1 cycle conditioner), and damaged treated ( 3 cycles conditioner) hair at $5 \mu \mathrm{m}$ scan sizes [LaTorre and Bhushan, 2005b]. 
virgin treated hair are quite comparable in surface roughness maps, examination of the treated hair surface shows an increase in friction force, especially in the area surrounding the scale edge bottom level. These frictional patterns observed in treated hair were not like anything observed in the virgin or chemically damaged cases. Images of all hair types have shown friction variation due to edge contributions and cuticle mechanical damage that has left only remnants of cuticle sublayer (such as the endocuticle). Further investigation of the corresponding treated hair roughness images showed this increase in friction was not due to a significant change in surface roughness, either. One explanation for the increase in friction force of treated hair on the micro/nanoscale is that during tip contact meniscus forces between the tip and the conditioner/cuticle become large as the tip rasters over the surface, causing an increase in the adhesive force. This adhesive force is of the same magnitude as the normal load, which makes the adhesive force contribution to friction rather significant. Thus, at sites where conditioner is accumulated on the surface (namely around the cuticle scale edges), friction force actually increases.

In general, friction forces are higher on chemically damaged hair than on virgin hair. Although friction forces were similar in magnitude, it was observed that the friction force on the cuticle surface of chemically damaged hair showed a much larger variance, which contributed to the higher friction values. Chemically damaged treated hair shows a much stronger affinity to the conditioner. It is widely known that the cuticle surface of hair is negatively charged. This charge becomes even more negative with the application of chemical damage to the hair. As a result, the positively charged particles of conditioner have even stronger attraction to the chemically damaged surface, which explains the increased presence of conditioner (and corresponding higher friction forces) 
when compared to virgin treated hair. With the application of three conditioner cycles on chemically damaged treated hair, friction force is still higher near the cuticle edge, however it is also increased all over the cuticle surface, showing a more uniform placement of the conditioner.

Figure 4.10 shows adhesive force maps for the various hair, which gives a measurement of adhesive force variation on the surface. Virgin treated hair shows a higher adhesive force than virgin hair due to the meniscus effects that come about from AFM tip interaction with the conditioner on the cuticle surface [Bhushan, 1999a, b, 2002]. The same trend is true and even more evident for chemically damaged treated hair compared to chemically damaged hair. A possible reason that one cycle of conditioner on chemically damaged hair showed higher average adhesive force than three cycles could be because the three cycles generally places the conditioner more uniformly on the surface rather than accumulating it most near the bottom surface near the cuticle edge, which is where the adhesive force maps were generally taken. Nevertheless, the increased adhesive force shown in the plots is a clear indication of conditioner present on the hair surface, and its localization can be observed.

Figure 4.11 presents surface roughness, coefficient of friction, and adhesive force plots for the various virgin and chemically damaged hair discussed above. The data is also presented in Table 4.2 [LaTorre and Bhushan, 2005b]. Surface roughness for human hair is characterized by a vertical descriptor, height standard deviation $\sigma$, and a spatial descriptor, correlation distance $\beta^{*}$ [Bhushan, 1999a, b, 2002]. Virgin and virgin treated hair showed similar $\sigma$ values, while $\beta^{*}$ was higher in virgin treated hair. Chemically damaged hair and both types of chemically damaged treated hair showed similar 
roughness values, although $\sigma$ was higher for the treated cases. The chemically damaged hair presented in this work is different from the chemo-mechanically damaged hair studied in LaTorre and Bhushan [2005a]. It seems that chemically damaging the surface does not lead to as much wear and surface roughness increase as the combination of both chemical and mechanical damage. Thus, it should be noted (and it is understandable) that there are differences between the chemo-mechanically and chemically damaged hair.

Coefficient of friction of virgin and virgin treated hair is similar, but slightly higher for the treated cases. Chemically damaged hair shows a much higher coefficient of friction, and with more variation in the values since the chemical damage varies throughout each individual fiber. An interesting finding was that, contrary to the virgin and virgin treated hair results, coefficient of friction for chemically damaged hair decreased with application of conditioner treatment (both one and three cycles). One possible explanation is that because the stronger negative charge on chemically damaged hair results in better attraction of conditioner, this leads to higher adhesive force but, more importantly, lower shear strength on the surface. This creates an overall effect of lubrication and ultimately lowers the coefficient of friction. 
Adhesive force maps for various hair

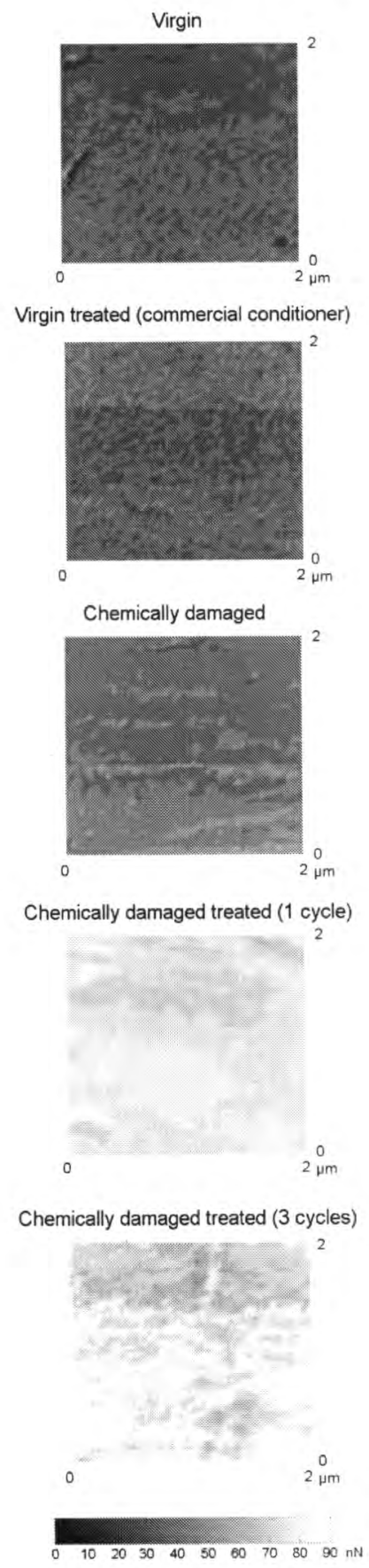

Fig. 4.10 Adhesive force maps displaying variations in adhesive force on the cuticle surface of various hair. [LaTorre and Bhushan, 2005b]. 
Surface roughness, coefficient of friction, and adhesive force data for various hair
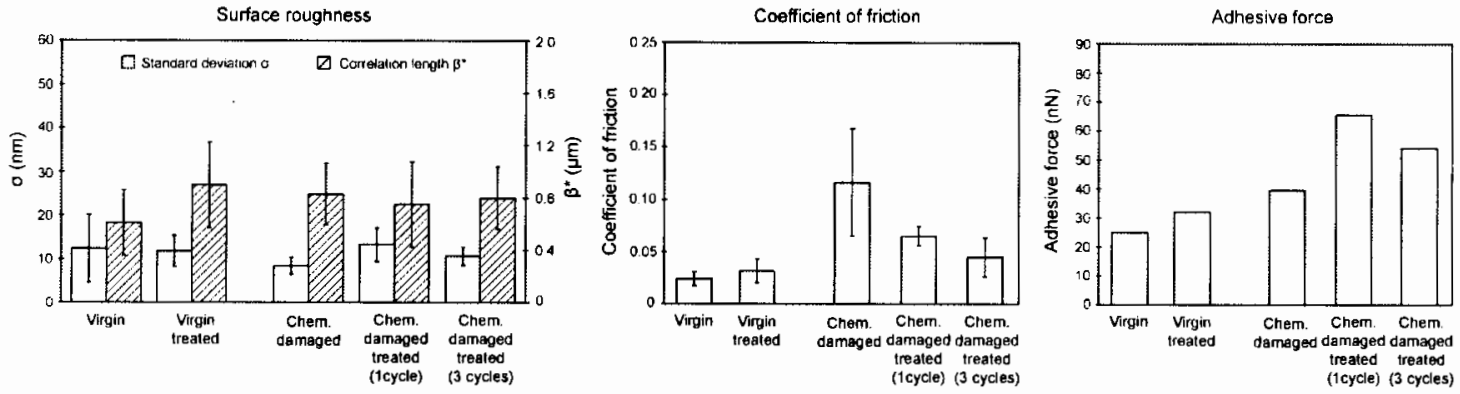

Fig. 4.11 Surface roughness, coefficient of friction, and adhesive force plots for various hair [LaTorre and Bhushan, 2005b].

\begin{tabular}{|c|c|c|c|c|}
\hline \multicolumn{5}{|c|}{$\begin{array}{c}\text { Surface roughness, coefficient of friction, and adhesive force for } \\
\text { virgin and chemically damaged hair, with and without conditioner } \\
\text { treatment }\end{array}$} \\
\cline { 2 - 5 } Hair type & Surface roughness & $\begin{array}{c}\text { Coefficient of } \\
\text { friction }\end{array}$ & $\begin{array}{c}\text { Adhesive } \\
\text { force }(\mathrm{nN})\end{array}$ \\
\cline { 2 - 5 } & $\sigma(\mathrm{nm})$ & $\beta^{*}(\mu \mathrm{m})$ & $0.02 \pm 0.01$ & $25 \pm 5$ \\
\hline Virgin & $12 \pm 8$ & $0.61 \pm 0.2$ & $0.03 \pm 0.01$ & $32 \pm 5$ \\
\hline $\begin{array}{c}\text { Virgin, } \\
\text { treated }\end{array}$ & $12 \pm 4$ & $0.90 \pm 0.3$ & $0.13 \pm 0.06$ & $39 \pm 0.5$ \\
\hline $\begin{array}{c}\text { Chemically } \\
\text { damaged }\end{array}$ & $8.4 \pm 2$ & $0.83 \pm 0.2$ & $0.05 \pm 0.02$ & $66 \pm 0.7$ \\
\hline $\begin{array}{c}\text { Damaged, } \\
\text { treated } \\
(1 \text { cycle })\end{array}$ & $13 \pm 4$ & $0.75 \pm 0.3$ & $0.04 \pm 0.02$ & $54 \pm 33$ \\
\hline $\begin{array}{c}\text { Damaged, } \\
\text { treated } \\
(3 \text { cycles })\end{array}$ & $11 \pm 2$ & $0.80 \pm 0.2$ & & \\
\hline
\end{tabular}

Table 4.2 Nanotribological parameters for virgin, chemically damaged, and treated hair. 


\subsubsection{Effect of relative humidity, temperature, and soaking, and durability measurements}

Figure 4.12 displays the effect of relative humidity on friction force and adhesive force. Coefficient of friction remained relatively constant for virgin and virgin treated hair. However, chemically damaged hair experienced a large increase in coefficient of friction at the high humidity, while chemically damaged treated hair experienced the opposite trend. This clearly shows that heavy moisture in the air plays a role on the frictional properties of chemically damaged hair. When combined with conditioner, a lubricating effect once again dominates as the water helps form a liquid layer which is more easily sheared. In terms of adhesive force, most samples showed a decrease in adhesive force with high humidity. It is expected that as water builds up on a surface, meniscus effects diminish and as a result do not readily contribute to adhesive force. Thus, the adhesive force is expected to come down at very high humidity.

Figure 4.13 displays the effect of temperature on friction force and adhesive force. The coefficient of friction generally decreased with increasing temperature. As the hair fiber heats up, conditioner which is present on the surface decreases in viscosity, causing a thinner film and lower friction force. The lower friction force ultimately leads to lower coefficient of friction values. Adhesive force was shown to decrease with increasing temperature as well. This was especially evident for treated hair fibers, whereas large adhesive force at room temperature decreased rapidly to adhesion values similar to nontreated fibers. It is most likely that at higher temperatures the thinning conditioner layer causes a reduced surface tension, which directly relates to the drop in adhesive force. 
Virgin, chemically damaged, and chemically damaged treated hair samples were soaked in de-ionized water for five minutes. Their corresponding coefficient of friction was measured and compared to coefficient of friction values for dry samples which were adjacent to the wet samples on the respective hair fiber. Figure 4.14 shows the results for two hair samples of each hair type. Virgin hair exhibits a decrease in coefficient of friction after soaking. Virgin hair is more hydrophobic (see Table 3.4), so more of the water is present on the surface and results in a lubrication effect after soaking. Chemically damaged hair tends to be hydrophilic due to the chemical degradation of the cuticle surface, and results in absorption of water after soaking. This softens the hair, which leads to higher friction, even with conditioner treatment. This is yet another indication that virgin and chemically damaged hair have significantly different surface properties which in many cases results in opposite trends for their nanoscale tribological properties. Adhesive force for virgin hair remained approximately the same before and after soaking, while it decreased for chemically damaged and chemically damaged treated hair after soaking.

Figure 4.15 shows the durability effects on friction force for various hair. Above the graph are pictures of unworn and worn virgin hair, with the cuticle edge serving as a reference point. Before testing, the surface is relatively smooth and void of any large debris or wear. After 1000 cycles at approximately $10 \mu \mathrm{N}$ load with a stiff silicon AFM tip, however, the interaction has caused degradation and wear (scratch) marks on the cuticle scale. This is the type of wear one could potentially see if hair were to come in contact with sand from a day spent at the beach, among other activities. Virgin hair shows an obvious increase in friction force signal as the scratch mark digs further into the 
surface. By this time the lubricious lipid layer on the surface of the virgin cuticle has been worn away and the friction force comes close to the magnitude of chemically damaged hair friction force at the onset of cycling. When conditioner is applied to the virgin hair, however, the wear does not show up as an increase in the friction force. Thus, conditioner serves as a protective covering to the virgin hair and helps protect the tribological properties when wear ensues. 


\section{Effect of relative humidity on \\ coefficient of friction and adhesive force}
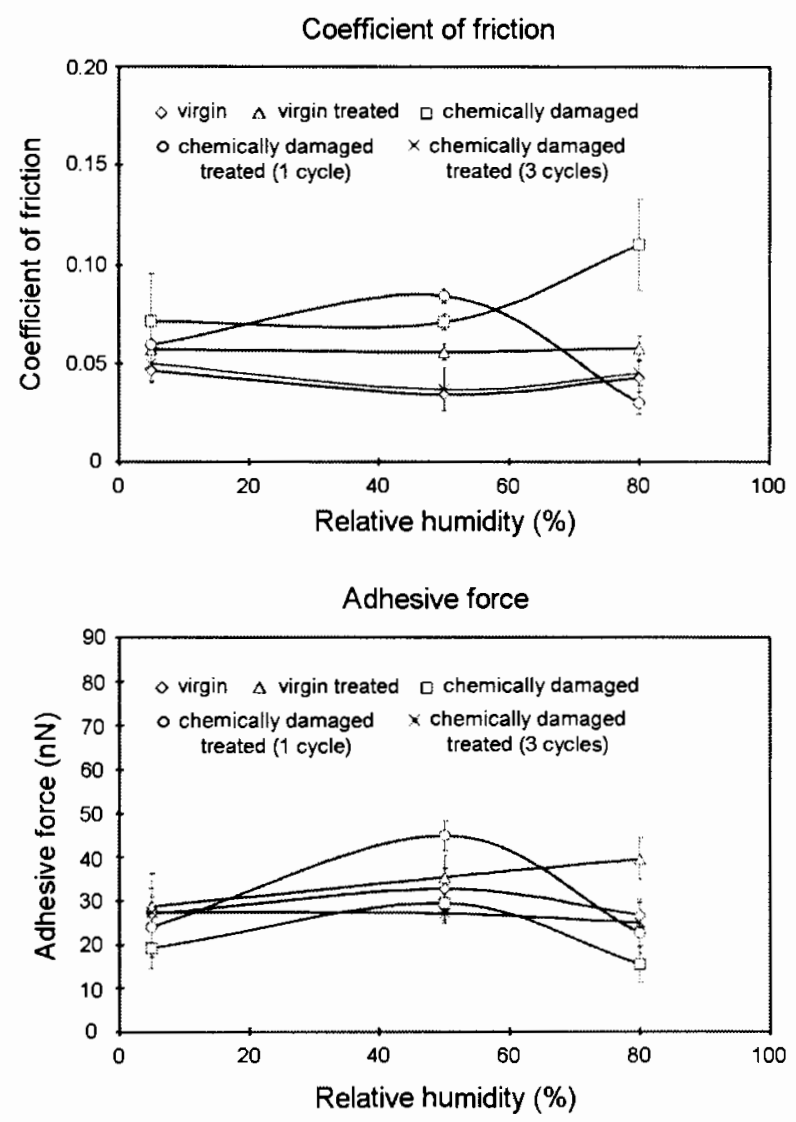

Fig. 4.12 Effect of relative humidity on nanotribological properties of various hair [LaTorre and Bhushan, 2005b]. 
Effect of temperature on

coefficient of friction and friction force
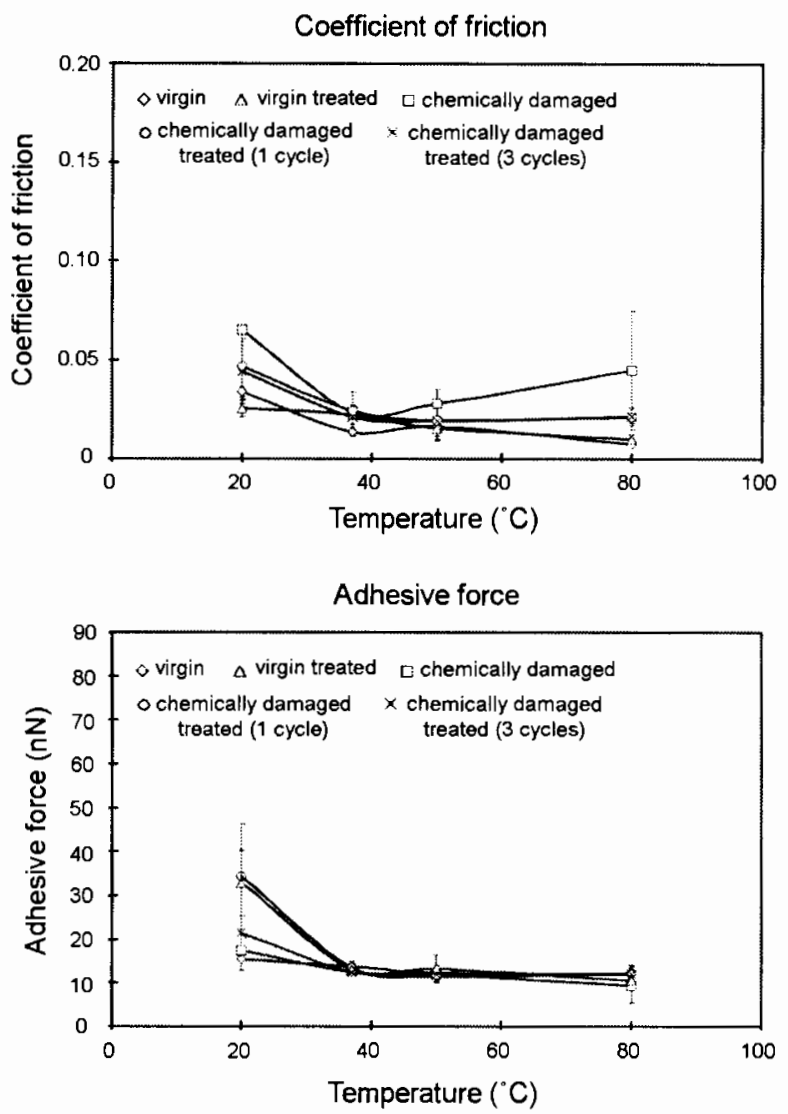

Fig. 4.13 Effect of temperature on nanotribological properties of various hair [LaTorre and Bhushan, 2005b]. 
Coefficient of friction
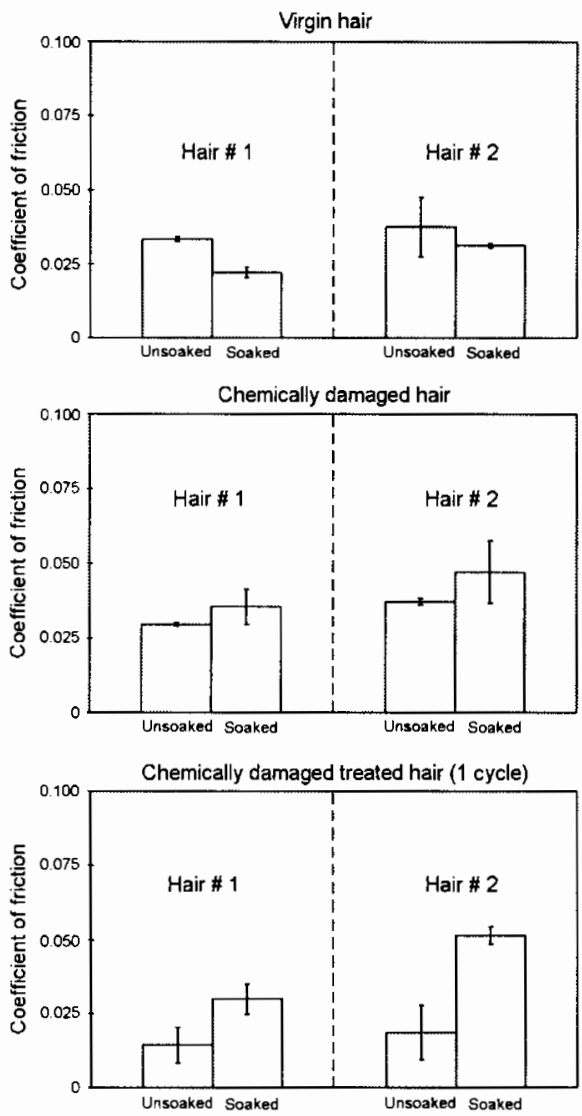

Adhesive force
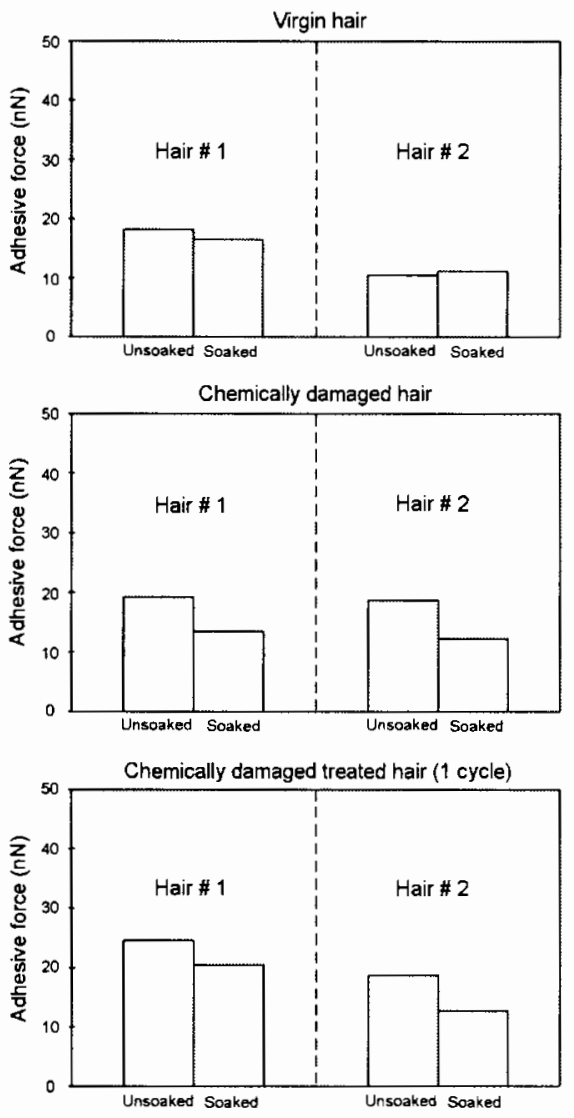

Fig. 4.14 Effect of soaking in de-ionized water on coefficient of friction and adhesive force for virgin, chemically damaged, and chemically damaged treated (1 cycle commercial) hair [LaTorre et al., 2006]. 
Surface roughness of unworn and worn virgin hair

Before

cycling

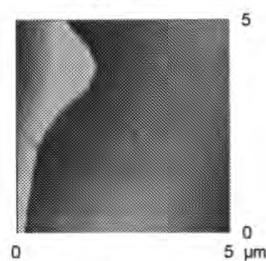

After 1000 cycles at $10 \mu \mathrm{N}$ load

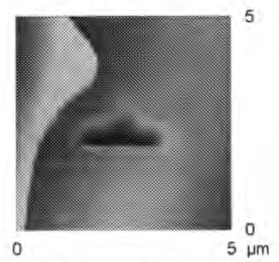

Durability effects on friction force of various hair

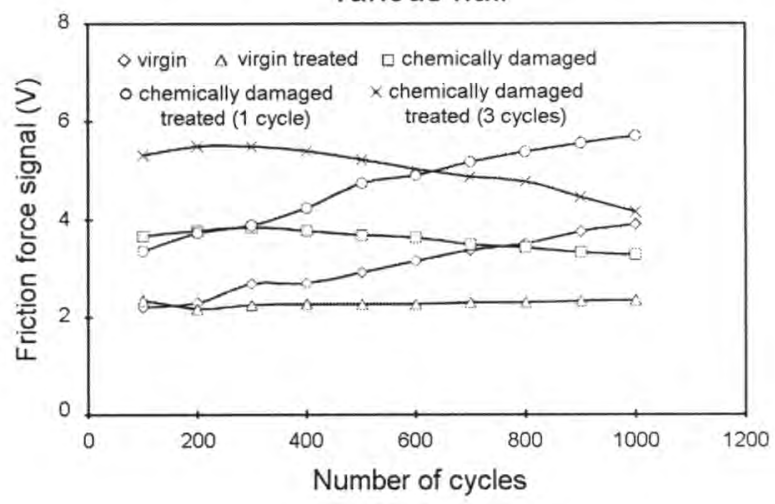

Fig. 4.15 Durability study of friction force change as a function of AFM tip cycling for various hair. The images above the plot signify before and after comparisons of a cuticle surface subjected to cycling at a $10 \mu \mathrm{N}$ load [LaTorre and Bhushan, 2005b]. 


\subsection{Surface roughness, friction, and adhesion for hair treated with various combinations of conditioner ingredients}

Figure 4.16 displays the representative surface roughness and friction force maps for chemically damaged hair and the seven different applied treatments (see Table 3.3). When conditioner is applied to the surface, a pattern of high friction is shown in the area surrounding the bottom edge of the cuticle. Likewise, the application of a BTMAC surfactant (with no silicone deposition) or a BAPDMA surfactant (no silicone deposition) results in similar friction features. This is believed to be an area of conditioner accumulation which causes increased friction due to meniscus effects. Friction maps for PDMS blend of silicone (at both low and high deposition levels) added to the BTMAC surfactant do not show this increase as readily, suggesting that this type of silicone is not a contributor of high friction force on the nanoscale. This can be due to the fact that a PDMS type silicone is fairly mobile on the surface and thus does not cause the same meniscus effects as the AFM tip rasters through it. Also, the surface energy of the PDMS silicone is believed to be lower than that of the cationic surfactants. As a result, the meniscus force affecting the friction between the AFM tip and silicones in the conditioner is lower than that of conditioner without silicones. Thus, the overall friction force will be lower. For high deposition levels of amino silicone, however, there is a high variation of friction force and a more distributed layer. The amino group typically is less mobile and harder to move, which accounts for a different slip plane flow that PDMS silicone.

Figure 4.17(a) displays the adhesive force maps for chemically damaged hair and the seven different treatments. As shown in the legend, a lighter area corresponds to a 
higher tip pull-off force (adhesive force). Chemically damaged untreated hair has relatively low adhesive force, and is more or less consistent over the hair surface. In nearly all cases, addition of a conditioner treatment caused an increase in meniscus forces, which in turn increased the adhesive pull-off force between the AFM tip and the sample. Observing the chemically damaged treated hair, the uneven distribution of the conditioner layer is seen. This uneven distribution is also most evident for the amino silicone images, in which the less mobile silicone brings about a distinguishable change in adhesion over the surface. For the PDMS blend silicone, it is seen that adhesion over the surface is much more consistent than the amino silicone, where various areas of high adhesion occur. 


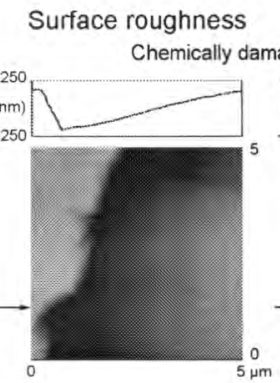

Friction force
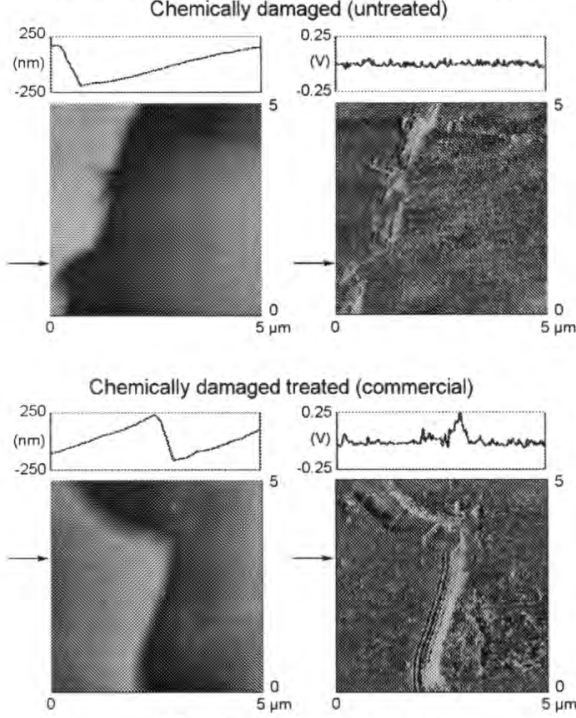

(BTMAC surfactant, no silicone)
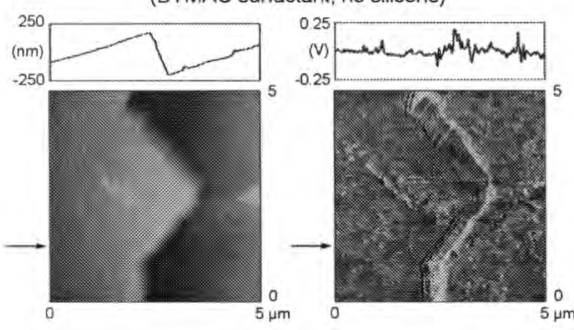

(PDMS blend silicone, low deposition)

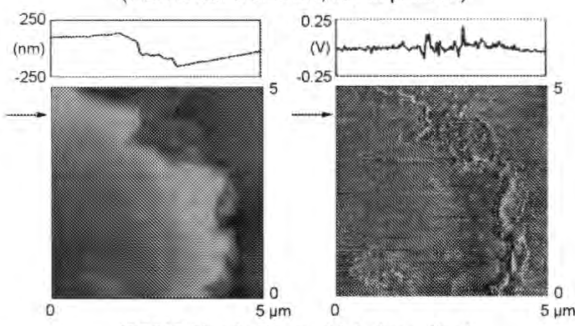

(PDMS blend silicone, high deposition)

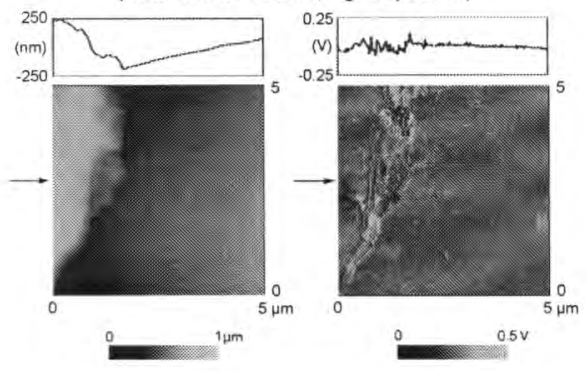

Surface roughness Friction force (Amino silicone, low deposition)
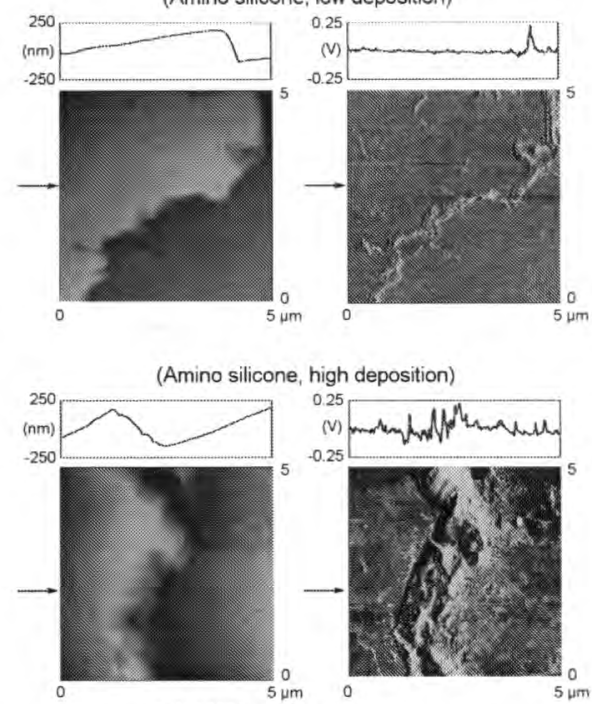

(BAPDMA surfactant, no silicone)
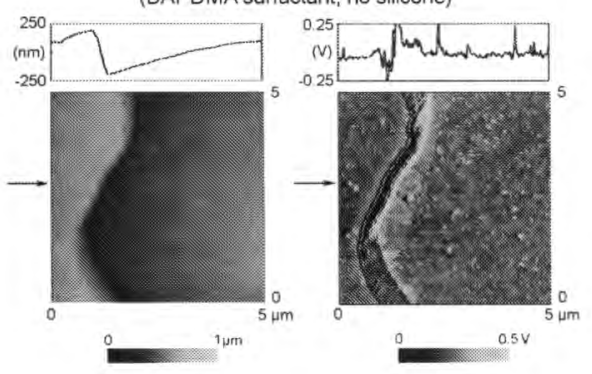

Fig. 4.16 Surface roughness and friction force maps of Caucasian chemically damaged hair with various treatments [LaTorre et al., 2006]. 
It is important to note that while adhesive force maps presented are representative images for each treatment, adhesive force varies significantly when treatments are applied to the hair surface. Figure 4.17(b) shows histograms of all adhesive force data for chemically damaged, chemically damaged treated, PDMS blend silicones (high deposition), and amino silicones (high deposition). Chemically damaged treated hair shows a much larger range of adhesive force values and a normal distribution, which suggests that the conditioner layer is normally distributed. The histogram for PDMS blend silicone treatment shows a normal distribution at the larger adhesive force values, but also shows another peak at low adhesion values. Amino silicone treated hair follows a normal distribution, but it is interesting to note the distinct groupings of the adhesion values and the spacing between them. This is further evidence that the amino silicone groups most likely attach immediately to the hair surface and are less mobile than PDMS silicone, causing distinct regions of high and low adhesion values over the cuticle surface. 
Adhesive force maps for chemically damaged hair with various treatments

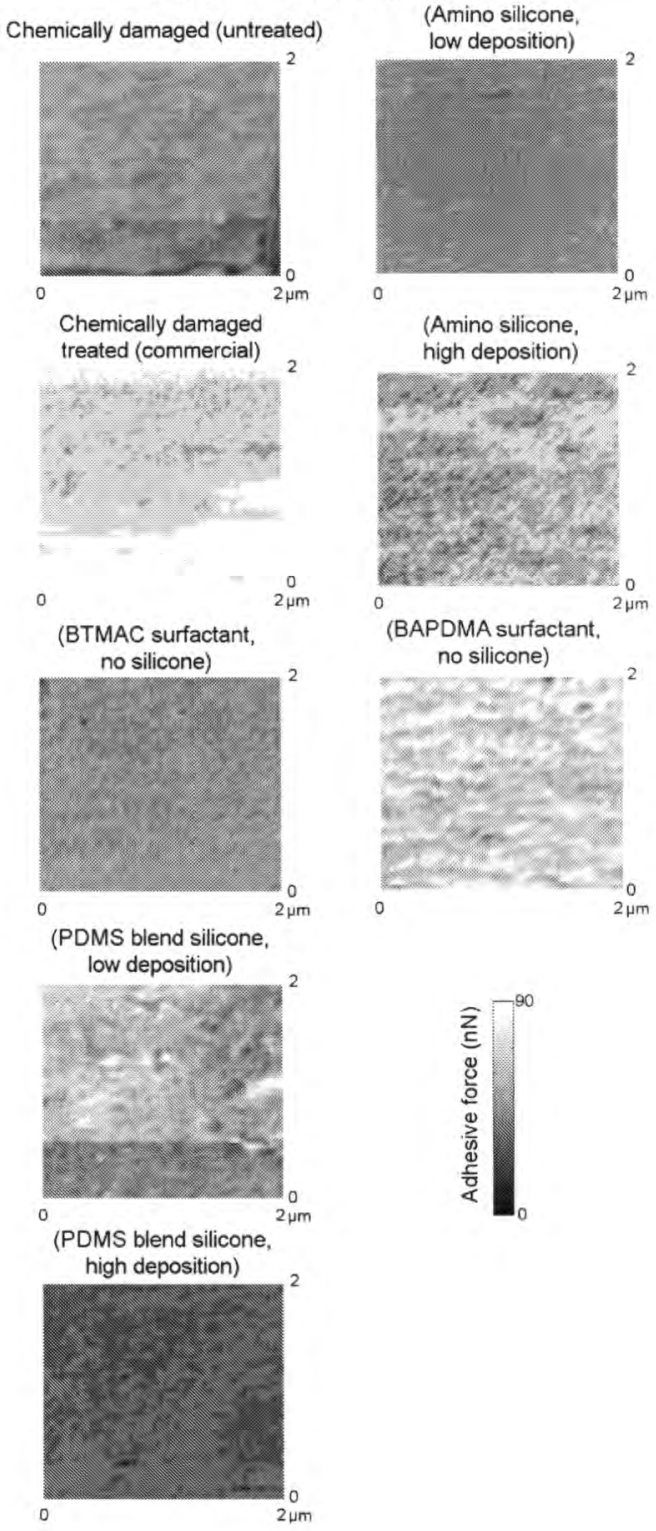

(a)

Continued

Fig. 4.17 (a) Adhesive force maps for damaged hair with various treatments (b) Adhesive force histograms for various hair [LaTorre et al., 2006]. 
Fig. 4.17 continued

Adhesive force histograms for various hair

Chemically damaged treated

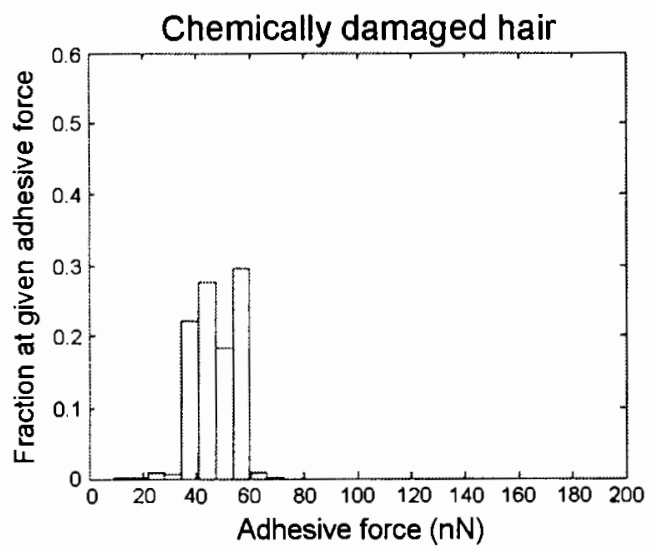

(commercial)
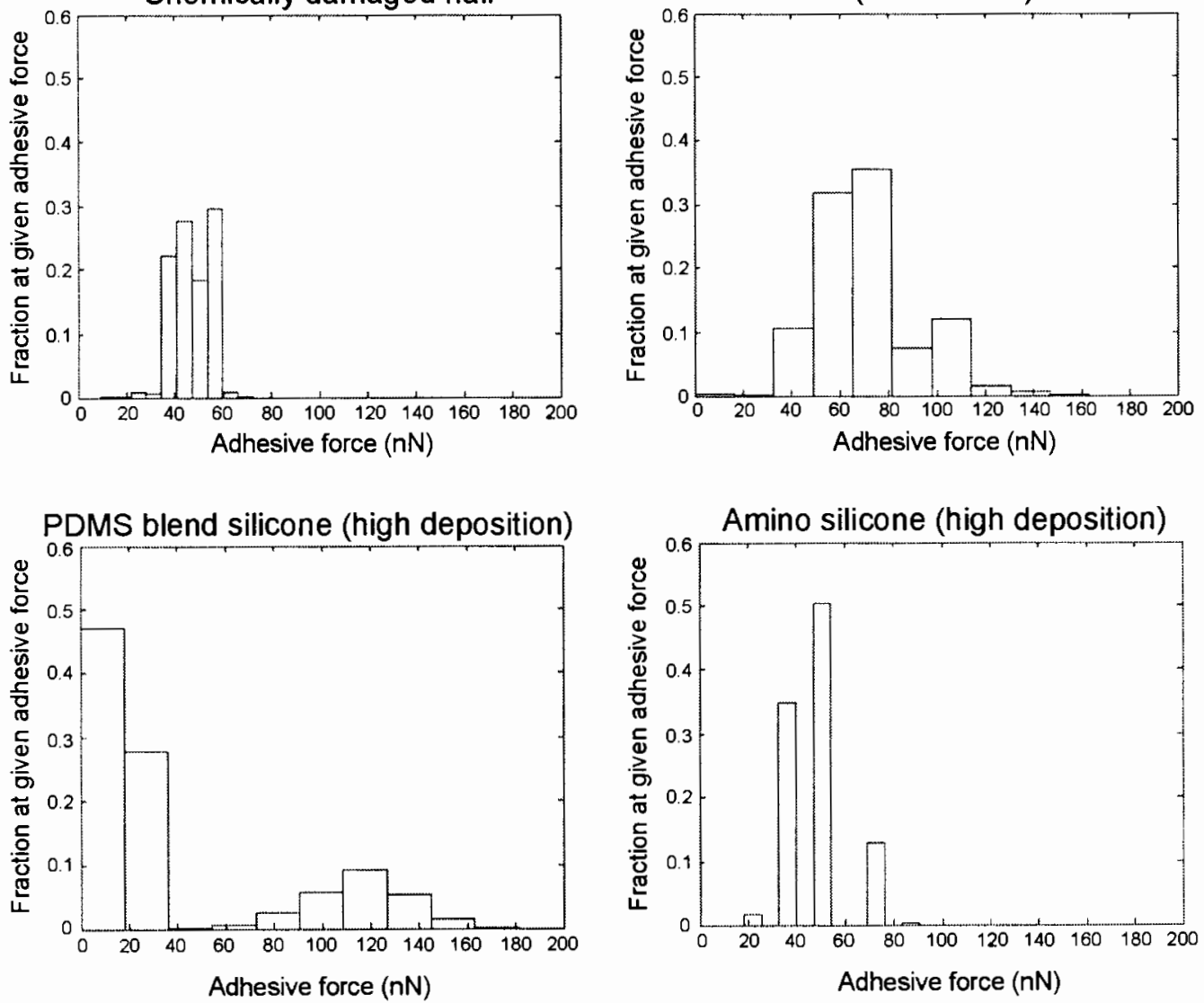

(b) 
Figure 4.18 displays a summary of the data collected for all chemically damaged hair samples and their treatments. Table 4.3 reviews some of the observations and corresponding mechanisms which help to explain the trends found [LaTorre et al., 2006]. The application of the commercial conditioner to the chemically damaged hair caused a decreased coefficient of friction and a large increase in adhesive force. The decreased coefficient of friction may be explained by the fact that the chemically damaged hair accumulates much of the positively charged conditioner on the surface due to its highly negative charge, which in turn makes it easier to shear the liquid on the surface, causing lower coefficient of friction. However, the nanoscale pull-off force (adhesive force) is much larger than on the untreated hair because of meniscus effects. In general, adhesive force varied widely, but typically showed a significant increase with the presence of conditioner. As discussed previously, this is a clear sign that meniscus effects are influencing the pull-off force between the tip and the sample.

In most cases, the macroscale and microscale coefficient of friction followed the same trend, in which a decrease was observed with the addition of the PDMS blend or amino silicones to the BTMAC surfactant. The silicones are typically used as a major source of lubrication and thus give the conditioner more mobility on the hair surface compared to just surfactants and fatty alcohols. The inverse trend was seen only for the amino silicone group. The amino silicones have a strong electrostatic attraction to the negatively charged hair surface, which in turn creates higher binding forces and less mobility. The dampened mobility of the amino silicone at high deposition levels, with respect to hair surface and tip, may account for this wide variation in coefficient of friction and large adhesive force values. 

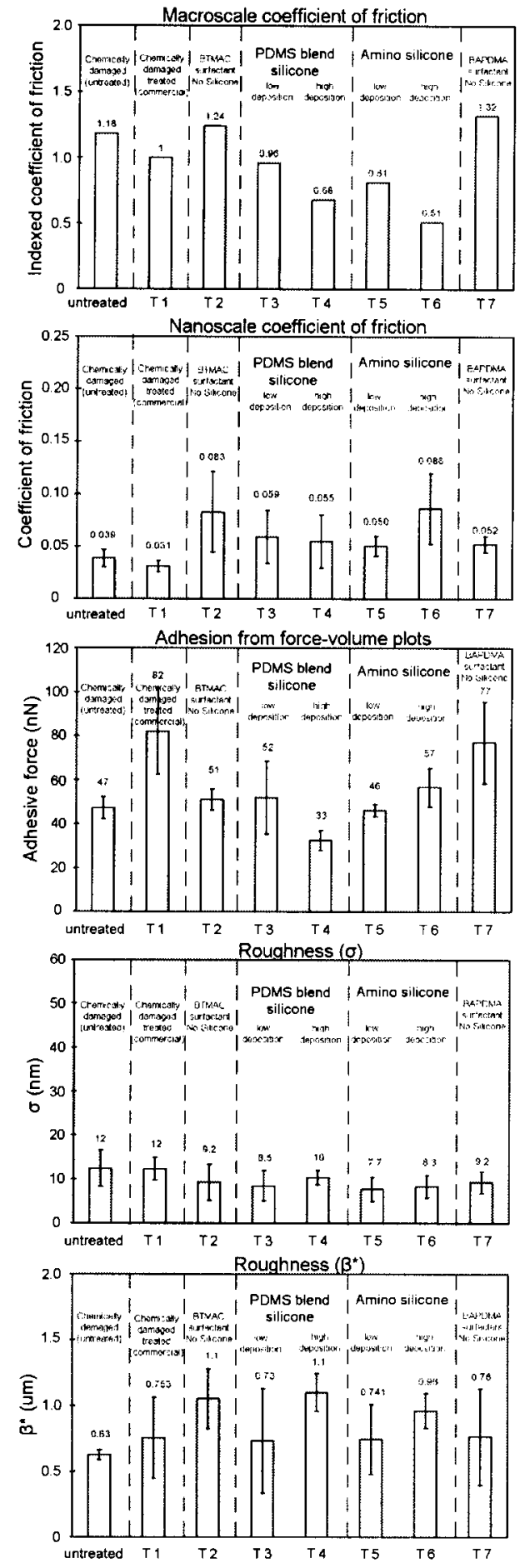

Fig. 4.18 Coefficient of friction, adhesive force, and surface roughness plots for damaged hair with various treatments [LaTorre et al., 2006]. 


\begin{tabular}{|c|c|}
\hline Observation & Mechanism \\
\hline \multicolumn{2}{|c|}{ Damaged vs. damaged treated hair } \\
\hline $\begin{array}{l}\text { Damaged hair shows a decrease in } \\
\text { coefficient of friction but an increase in } \\
\text { adhesion from the application of } \\
\text { commercial conditioner }\end{array}$ & $\begin{array}{l}\text { The conditioner layer deposited on the } \\
\text { surface of the damaged hair results in a } \\
\text { lower shear strength which in turn lowers } \\
\text { the coefficient of friction, while meniscus } \\
\text { effects increase the pull-off (adhesive) } \\
\text { force between the tip and hair sample. }\end{array}$ \\
\hline \multicolumn{2}{|c|}{ PDMS blend vs. amino silicone (at high deposition) } \\
\hline $\begin{array}{l}\text { Amino silicones interact strongly with } \\
\text { negatively charged hair surface, especially } \\
\text { at high deposition levels. }\end{array}$ & $\begin{array}{l}\text { A stronger electrostatic attraction exists } \\
\text { which results in stronger binding forces } \\
\text { (which leads to higher adhesion) for high } \\
\text { deposition amino silicone. }\end{array}$ \\
\hline $\begin{array}{l}\text { Amino silicone thickness distribution on } \\
\text { hair is less uniform than with PDMS blend. }\end{array}$ & $\begin{array}{l}\text { Less mobility with amino silicones, so } \\
\text { molecules attach to hair at contact and do } \\
\text { not redistribute easily. }\end{array}$ \\
\hline \multicolumn{2}{|c|}{ Surfactant vs. surfactant plus addition of silicone } \\
\hline $\begin{array}{l}\text { Adhesion remained approximately the } \\
\text { same while coefficient of friction decreased } \\
\text { with addition of PDMS blend silicone and } \\
\text { amino silicone (low deposition). } \\
\text { Coefficient of friction remained about same } \\
\text { at high deposition amino silicone. }\end{array}$ & $\begin{array}{l}\text { Mobility becomes easier with addition of } \\
\text { PDMS blend silicone which leads to lower } \\
\text { coefficient of friction. At high deposition } \\
\text { of amino silicones, mobility ceases and } \\
\text { coefficient of friction becomes high again. }\end{array}$ \\
\hline \multicolumn{2}{|c|}{ BTMAC surfactant vs. BAPDMA surfactant } \\
\hline $\begin{array}{l}\text { BTMAC surfactant has lower adhesion but } \\
\text { higher coefficient of friction than } \\
\text { BAPDMA surfactant. }\end{array}$ & $\begin{array}{l}\text { Interaction of surfactants with damaged } \\
\text { hair surface causes inherent differences in } \\
\text { coefficient of friction and adhesion. } \\
\text { BAPDMA has both amino and amine } \\
\text { groups which increases polarity. }\end{array}$ \\
\hline
\end{tabular}

Table 4.3 Observations and corresponding mechanisms regarding coefficient of friction and adhesion for various hair treatments. 
In terms of adhesive force, it was previously observed in Figure 4.17(a) that the amino silicone treatments showed much more distinct regions of higher and lower adhesion compared to PDMS blend silicones. This nonuniform amino silicone thickness distribution on hair is also believed to be caused by the inhibited mobility, as the molecules immediately attach to the hair at contact and do not redistribute as a uniform coating. The increased polarity of the amino silicones compared to the PDMS blend can also be a major contributor of the higher friction and adhesion at high deposition levels.

If looking at the hair with only the BTMAC surfactant and fatty alcohols, and then adding low deposition levels of PDMS blend (with high MW weight) or amino silicone, it is seen that coefficient of friction decreases, while adhesion remained approximately the same. If the deposition level is increased, it is observed that the PDMS blend is still lower, but now the coefficient of friction for the amino silicone is about the same as the BTMAC only samples. The mobility of the conditioner layer is again a major issue, as this easier mobility accounts for lower coefficient of friction. However, as is seen when there is a large amount of the amino silicones on the hair surface, the mobility ceases and the coefficient of friction becomes high again.

The BAPDMA surfactant typically showed a higher adhesive force than the BTMAC surfactant. However, there may be an increased accumulation of the BAPDMA surfactant such that the conditioner layer is more easily sheared by the tip, as is the case for chemically damaged treated hair. The inherent differences in surfactant composition and how they interact with the chemically damaged hair surface most likely account for the coefficient of friction differences. 
In respect to roughness, the vertical standard deviation decreased slightly with most treatments, although standard deviations were similar. The spatial parameter increased slightly with treatments, but the variation also becomes extremely high.

\subsection{Investigation of directionality dependence and scale effects on friction and}

\section{adhesion of hair}

\subsubsection{Directionality dependence}

Figure 3.2, shown previously, displays friction test apparatuses used for macroand micro/nanoscale coefficient of friction measurements, as discussed earlier. The macroscale friction test apparatus, previously discussed, consists of taking a hair fiber switch and running a block of synthetic skin over the fibers. AFM coefficient of friction measurements take place on a single fiber. With the microscale AFM tip ( $4 \mu \mathrm{m}$ radius), several cuticle scales may come into contact at the same time depending on their length, causing the scale edges to become a significant factor in the resulting coefficient of friction values. This is especially evident when scanning in the tip-to-root direction of the hair (referred to as "against cuticle"). With the nanoscale AFM tip (30-50 nm radius), single asperity contact can be simulated, and the cuticle edges can be avoided by limiting the scan area to a small section of the surface.

Figure 4.19 displays SEM micrographs and AFM height maps of virgin hair at various scales. The top SEM micrograph shows the hair fiber so that the full diameter is observed. During macroscale friction testing, many hair fibers will contact the upper sample (generally synthetic skin) at the same time. The next micrograph reveals better magnification of the cuticle scales. The scan length here is representative of that used for microscale coefficient of friction measurements. The $20 \times 20 \mu \mathrm{m}^{2}$ AFM height map and 


\section{Comparison of hair surface at different scales}

SEM micrographs
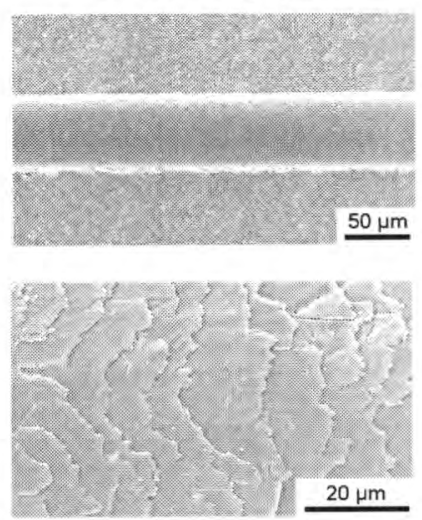

AFM height maps
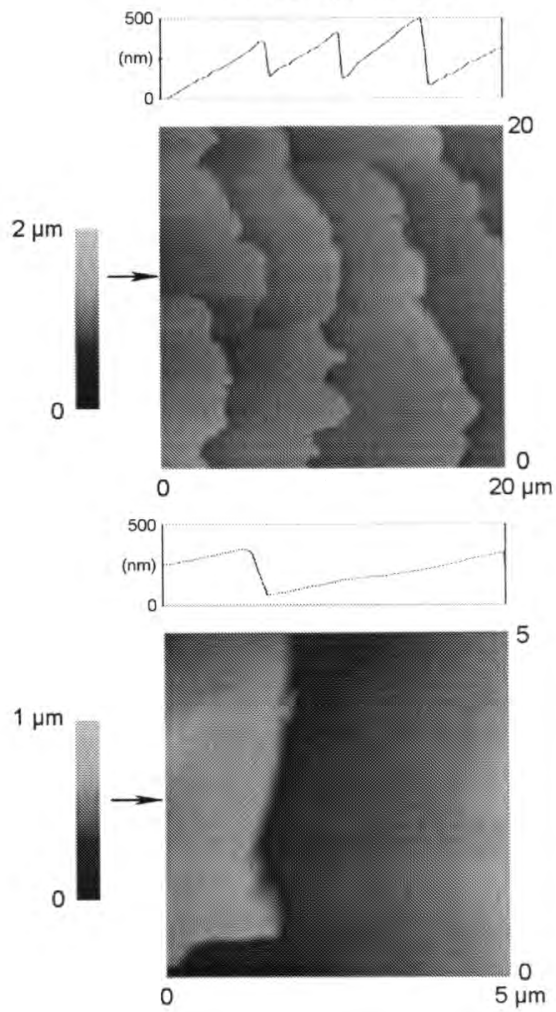

Fig. 4.19 Comparison of hair topography at different scales. 
corresponding cross-sectional plot show that cuticle scale heights vary from layer to layer, and range from approximately 300 to $500 \mathrm{~nm}$. With a $5 \times 5 \mu \mathrm{m}^{2}$ map we can focus on one cuticle scale edge. Most visible cuticle scale lengths are between 5 and $10 \mu \mathrm{m}$. For nanoscale coefficient of friction measurements, a $2 \mu \mathrm{m}$ scan length is generally used so as to avoid these scale edges affecting the data. However, if directionality dependence is to be studied, a $5 \mu \mathrm{m}$ scan line is sufficient.

Human hair has been shown in the past to have a directionality friction effect on the macroscale, making it easier to travel over the hair surface from root-to-tip than in the opposite direction due to the shingle-like orientation of cuticles [Robbins, 1994]. The outer surface of human hair is composed of numerous cuticle scales running along the fiber axis, generally stacked on top of each other. As previously discussed, the heights of these step changes are approximately $300 \mathrm{~nm}$. These large changes in topography make the cuticle an ideal surface for investigating the directionality effects of friction. The first row of Figure 4.20(a) displays a low resolution SEM micrograph and a friction profile for macroscale coefficient of friction measurements. Note that this data is taken for measurements where multiple fibers are in contact with the synthetic skin upper specimen at the same time, and does not correspond directly with the SEM micrograph. With an applied normal load of $50 \mathrm{mN}$ and $3 \mathrm{~mm}$ travel, it is observed that the friction force produced when scanning from root-to-tip (referred to as "along cuticle") is lower than that when scanning against cuticle. This is a direct consequence of the literally thousands of scale edges which come in contact with the synthetic skin. When traveling against cuticle, these edges act as tiny resistors to motion as they are forced backwards and uplifted from their interface with the underlying cuticle layers. The resistance to motion 
of so many cuticle edges at the same time becomes "additive" and results in higher values of friction, corresponding to higher coefficient of friction than when traveling along cuticle. For along cuticle travel, these edges are forced downward against the underlying cuticle layers so that the resistance effect of these edges is limited, which results in lower friction values.

The second row of Figure 4.20(a) shows an AFM height map corresponding to the microscale friction profile shown to its right. Due to the size of the hair, it was only possible to capture a rectangular height map as shown. It is evident that the $100 \mu \mathrm{m}$ travel results in the involvement of several cuticle scales. The applied normal load for the microscale friction profile (about $20 \mathrm{nN}$ ) is significantly reduced from that of the macroscale value, which consequently yields much lower friction forces. In the along cuticle direction, we can see small fluctuations in the friction data over the scan distance. These are caused by local variations in surface roughness, system noise, and changes due to traveling over the scale edges. However, when scanning against the cuticle, distinctly large spikes in the data are observed at roughly $5-10 \mu \mathrm{m}$ intervals. This is clearly the effects of the scale edges coming in contact with the AFM microscale tip and causing local collisions and ratcheting of the tip. Because of the sign convention of the AFM that causes a reversal in the sign when traversing the opposite direction, this signal is now observed to be highly negative. These edge effects are hence the primary item responsible for the higher friction and coefficient of friction observed in the against cuticle direction.

Directionality effects on nanoscale hair friction have previously been reported by LaTorre and Bhushan [2005a]. As shown in the bottom row of Figure 4.20(a), the scan size of $5 \times 2.5 \mu \mathrm{m}^{2}$ provides one cuticle scale edge to be studied (the height map is 
rectangular only to be consistent with the microscale image in the middle row). As the tip rasters in the along cuticle direction, a small decrease in the friction force is observed as the tip follows the scale edge on a downward slope. When the tip comes back in the against cuticle direction, colliding with the scale edge and climbing up the sharp peak results in a high friction signal. As discussed above, because of the sign convention of the $\mathrm{AFM} / \mathrm{FFM}$ that causes a reversal in the sign when traversing the opposite direction, this signal is now observed to be highly negative. The interesting difference between the two profiles lies in the fact that the magnitude of the decrease in friction when going up the step is much larger than the magnitude of the friction when the tip is going down the step, yet both signals are in the same direction. It is thus shown that the cuticle edge provides a local ratchet and collision mechanism that increases the friction signal at that point and clearly shows the directionality dependence caused by edge effects.

Coefficient of friction data showing directionality dependence on the macroscale for various virgin, damaged, and treated hair can be found in Bhushan et al. [2005]. Figure $4.20(\mathrm{~b})$ and Table 4.4 show a summary of the microscale coefficient of friction data for the various hair samples of this study. In most cases, the coefficient of friction has more than doubled when scanning in the against cuticle direction. A more in depth discussion on the coefficient of friction trends between the different hair types will follow. For now, however, it is important to realize that there is strong directionality dependence on coefficient of friction data for hair, especially on the microscale. On the nanoscale, while directionality dependence of friction force has been studied, actual coefficient of friction data is generally only measured on a small scan area which does not include the cuticle edge, so that only the true cuticle surface is involved [LaTorre and 
Bhushan, 2005a]. Hence, nanoscale coefficient of friction directionality data similar to Figure $4.20(b)$ is not shown. 
Directionality effects of hair friction on various scales
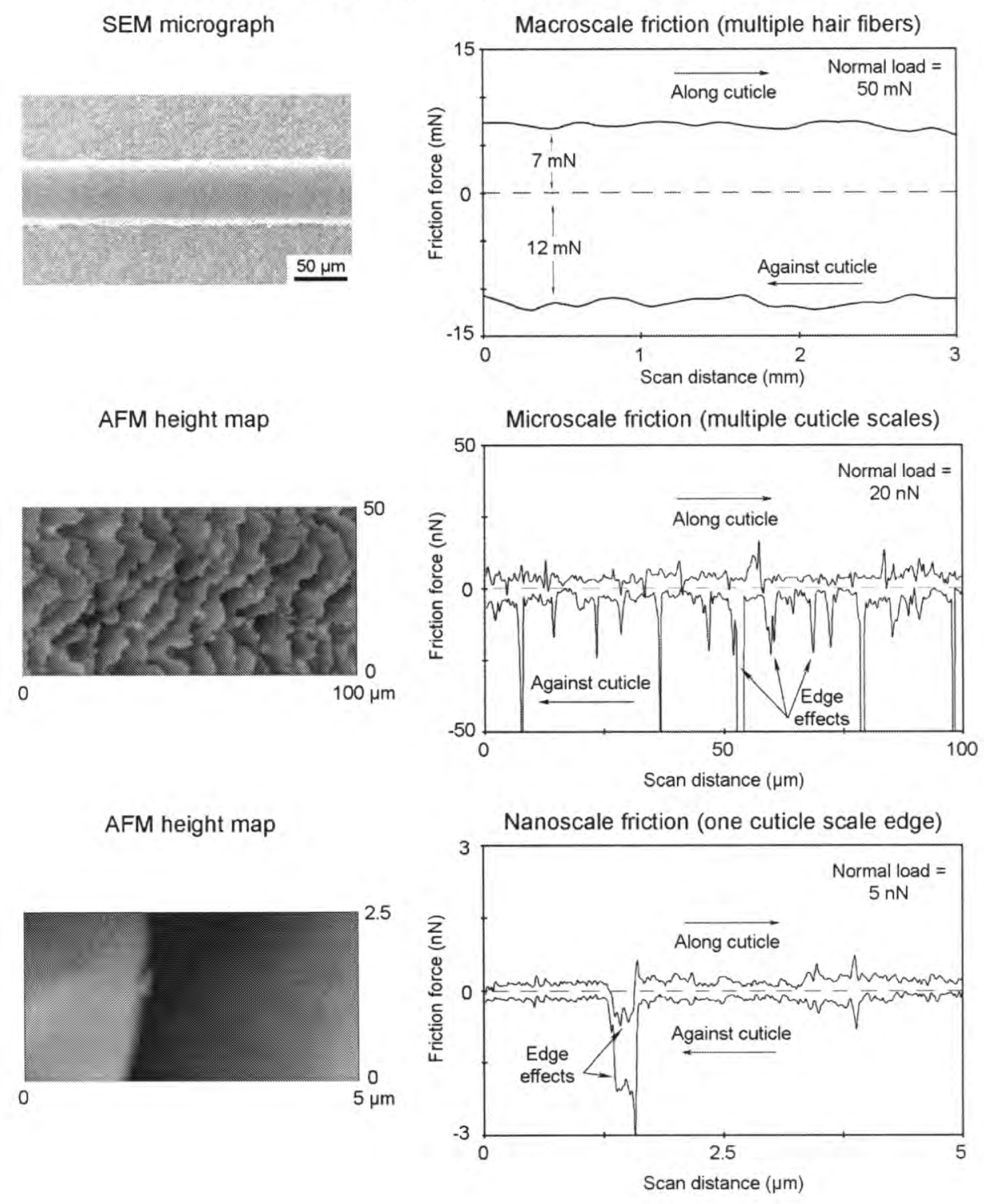

(a)

Continued

Fig. 4.20 (a) Directionality effects of hair friction on various scales (b) Microscale coefficient of friction data for various hair showing directionality effects. 
Fig. 4.20 continued

Directionality effects of friction on the microscale

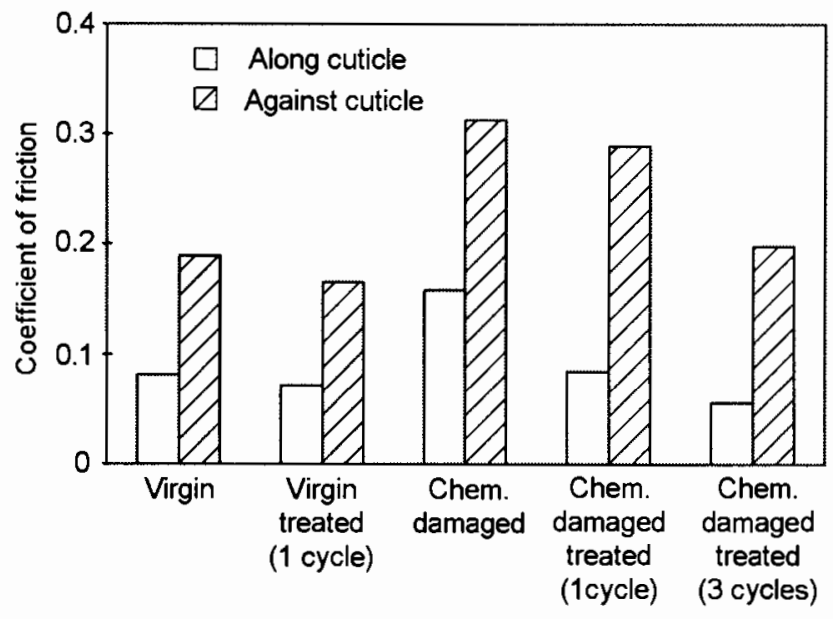

(b) 


\begin{tabular}{|l|c|c|}
\hline \multirow{2}{*}{ Hair sample } & \multicolumn{2}{|c|}{ Microscale coefficient of friction } \\
\cline { 2 - 3 } & Along cuticle & Against cuticle \\
\hline Virgin (untreated) & 0.08 & 0.19 \\
\hline Virgin treated (1 cycle commercial conditioner) & 0.07 & 0.17 \\
\hline Chemically damaged (untreated) & 0.16 & 0.31 \\
\hline $\begin{array}{l}\text { Chemically damaged treated (1 cycle commercial } \\
\text { conditioner) }\end{array}$ & 0.08 & 0.29 \\
\hline $\begin{array}{l}\text { Chemically damaged treated (3 cycle commercial } \\
\text { conditioner) }\end{array}$ & 0.06 & 0.20 \\
\hline
\end{tabular}

Table 4.4 Coefficient of friction and adhesive force values showing directionality dependence on the microscale. This dependence has also been observed on the nano- and macroscale.

\subsubsection{Scale effects}

Given the fact that the "directionality effect" is now observed to be universal for all types of hair and on all scales, and since the along cuticle direction is more relevant to our daily life (i.e. combing), we now focus on coefficient of friction data along cuticle. As described earlier, microscale and nanoscale coefficient of friction is taken as the slope of the least squares fit line of a friction force vs. normal force data curve. Figure 4.21(a) shows these types of raw data curves for various representative hair samples using both microscale (top plot) and nanoscale (bottom plot) AFM tips. The nanoscale data was taken from raw data used in LaTorre and Bhushan [2005b]. One can see a relatively linear relationship between the data points for each type of hair sample. If the least squares fit lines of Figure 4.21(a) are extended to intercept the horizontal axis (indicated 
by the dotted lines), then a value for adhesive force can also be calculated, since friction force $F$ is governed by the relationship of Equation 1. These adhesive force values serve as average adhesive force values over the course of the full scan profile, and differ slightly from the force calibration plots of Figure 4.21(b). From Figure 4.21(a), it is observed in both plots that treated hair fibers, whether virgin or chemically damaged, have much higher average adhesive force values as compared to their untreated counterparts. Chemically damaged hair is observed to have the highest coefficient of friction (highest slope) on both the micro- and nanoscales. As explained in LaTorre and Bhushan [2005a, b] and LaTorre et al. [2006], chemical damage to the hair causes the outer lubricious layer of the cuticle to wear off, resulting in an increased coefficient of friction.

Force calibration plots yield adhesive force values at a single point and are considered to be more relevant for measurement of the pull-off force between the tip and hair surface. Since it has been previously shown that treating both virgin and chemically damaged hair with conditioner results in large increases in adhesive force using both micro- and nanoscale AFM tips, we will focus only on the force calibration plots of the virgin and virgin treated hair to discuss mechanisms for this trend. The first plot in Figure 4.21(b) shows a typical virgin hair force calibration plot with the microscale AFM tip. The cantilever deflection is plotted on the vertical axis against the Z-position of the piezo scanner. As the piezo extends (from right to left in the graph), it approaches the tip and does not show any deflection while in free air. The tip then approaches within a few nanometers (point $\mathrm{A}$ ) and becomes attached to the sample via attractive forces at very close range (point B). After initial contact, any extension of the piezo results in a further 
deflection of the cantilever and tip. Once the piezo reaches the end of its designated ramp size, it retracts to its starting position (from left to right). The tip goes beyond zero deflection and enters the adhesive regime. At point $\mathrm{C}$, the elastic force of the cantilever becomes equivalent to the adhesive force, causing the cantilever to snap back to point $D$. The horizontal distance between A and D multiplied by the stiffness of the cantilever results in a quantitative measure of adhesive force [Bhushan, 1999a, b, 2002, 2005]. We can see that on the application of 1 cycle conditioner treatment to the virgin hair, the microscale adhesive force jumps to about $230 \mathrm{nN}$. This is clearly the effects of meniscus forces brought about by the presence of the conditioner layer on the cuticle surface which interacts with the tip [LaTorre and Bhushan, 2005a, b; Chen and Bhushan, 2005b]. With the nanoscale tip (bottom row of Figure 4.21(b)), increase in adhesive force is again seen with conditioner treatment.

It is important to notice that that adhesive force values on the microscale are always larger than those on the nanoscale for a given hair. To explain the scale dependency of adhesive force, we can model the hair-conditioner-tip interaction as a sphere close to a surface with a continuous liquid film [Chen and Bhushan, 2005b]. The adhesive force $F_{a}$, is the force needed to pull the sample away from the tip (which is the same as the adhesive force calculated with force calibration plots). $F_{a}$ is the sum of van der Waals force $F_{v d w}$ mediated by adsorbed water or conditioner layer and the meniscus force $F_{m}$ due to Laplace pressure $\left(F_{a}=F_{v d w}+F_{m}\right)$. The meniscus force $F_{m}$ is given by

$$
F_{m}=2 \pi R \gamma(1+\cos \theta)
$$


where $R$ is the tip radius, $\gamma$ is the surface tension of the conditioner, and $\theta$ is the contact angle between the tip and conditioner [Bhushan, 2002]. The increase in adhesive force calculated by force calibration plots with the microscale AFM tip compared to the nanoscale AFM tip is in large part due to the increased radius $R$ of the microscale ball, which consequently induces larger $F_{m}$.

Figure 4.22 and Table 4.5 display the coefficient of friction and adhesive force data on macro-, micro-, and nanoscales. Scale dependence is clearly observed. Macroscale data for virgin and virgin treated hair were taken from Bhushan et al. [2005]. The values for other hair were taken in part from the indexed coefficient of friction values in LaTorre and Bhushan [2005b] which were transformed into actual values (as described previously). No adhesive force data is presented for the macroscale data because the adhesive force contribution to friction is considered to be negligible compared to the applied normal load. On the micro- and nanoscales, however, the magnitude of the adhesive force is the same as that of the applied normal load, so they have significant contributions on the coefficient of friction data, and thus are presented. Microscale values are taken from the raw data represented in Figure 4.21(a) and (b). Nanoscale data was taken from LaTorre and Bhushan [2005b]. 
Friction force vs. normal load curves for

micro- and nanoscale coefficient of friction

Microscale friction force vs. normal load curves

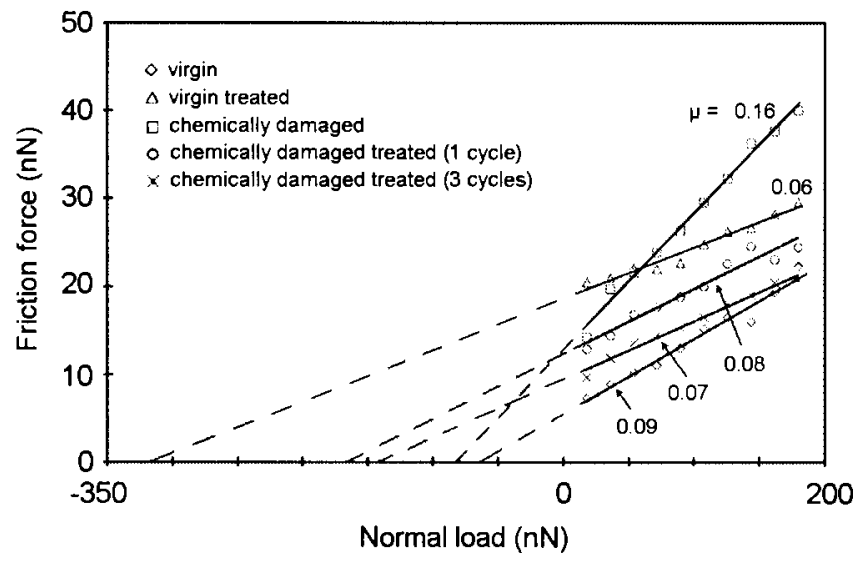

Nanoscale friction force vs. normal load curves

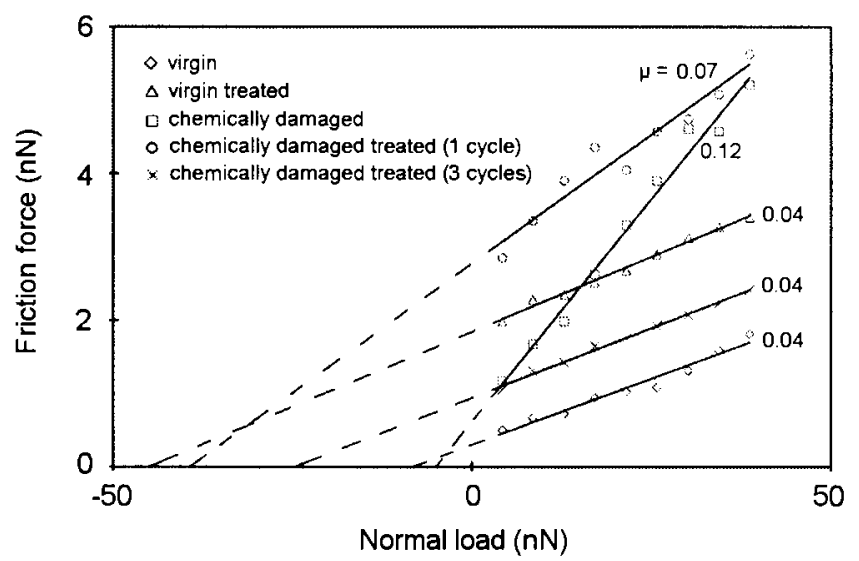

(a)

Continued

Fig. 4.21 (a) Friction force vs. normal force curves for micro- and nanoscale coefficient of friction (b) Adhesive force comparison of virgin and virgin treated hair using microand nanoscale AFM tips and force calibration plot technique. 


\section{Fig. 4.21 continued}

Adhesive force comparison of virgin and virgin treated hair using micro- and nanoscale AFM tips and force calibration plot technique

Microscale force calibration plots
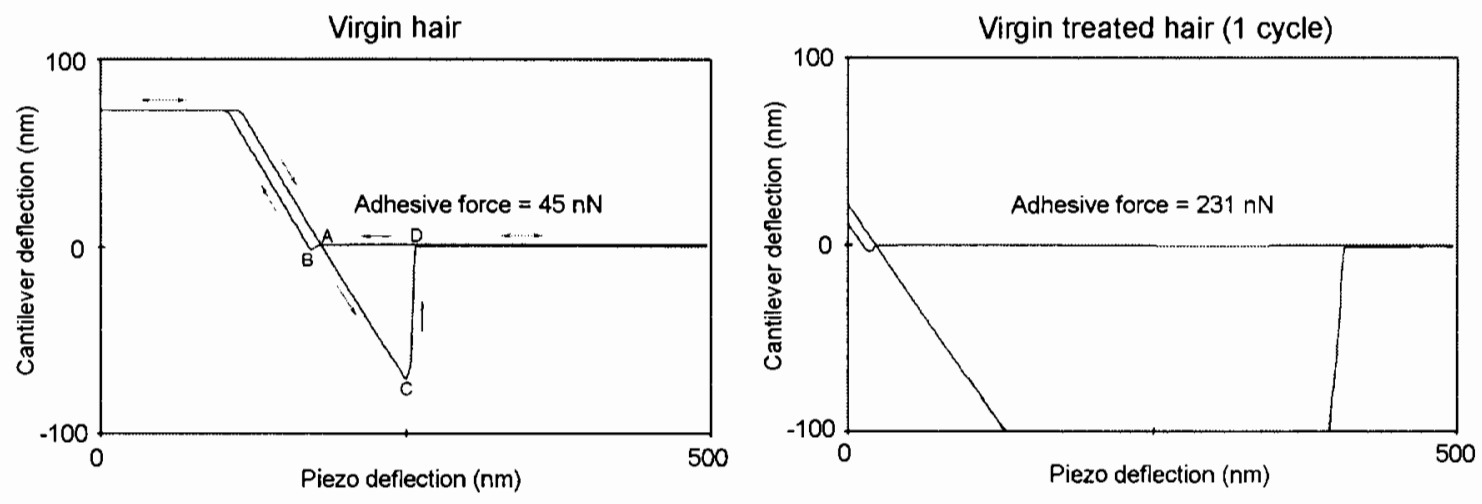

Nanoscale force calibration plots
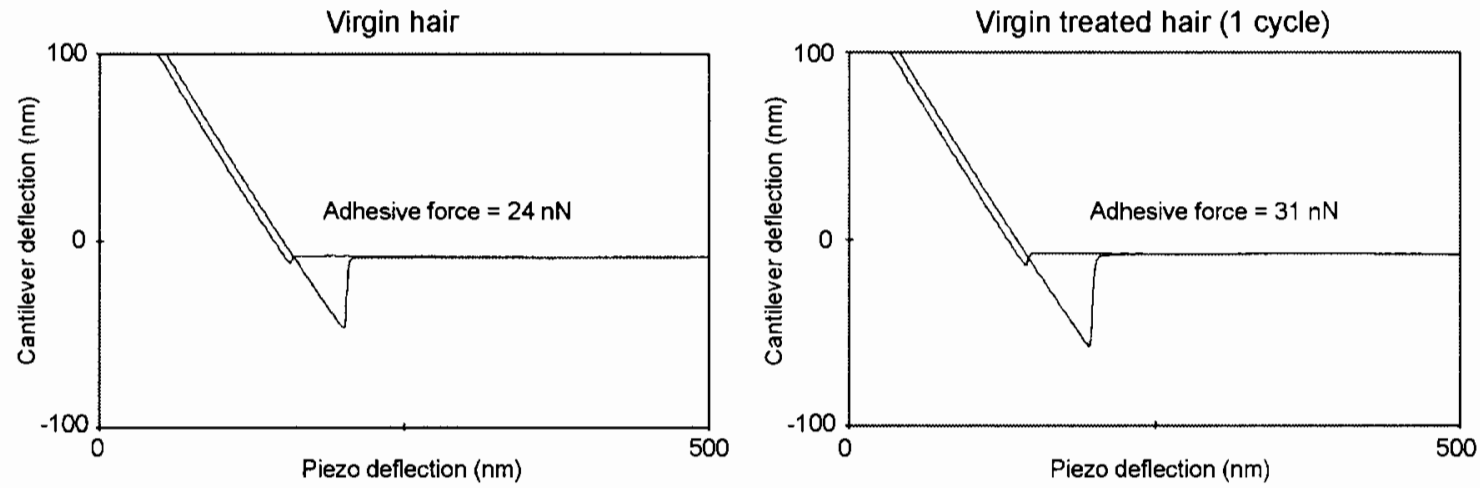

(b) 
Macroscale coefficient of friction (COF) data is shown in the top row of Figure 4.22: Chemically damaged hair has higher coefficient of friction than virgin hair, 0.24 compared to 0.14 . Coefficient of friction decreases with the application of 1 cycle of conditioner for both virgin and chemically damaged hair. When 3 cycles of conditioner are applied to chemically damaged hair, there is only a slight decrease compared to 1 cycle application, 0.14 to 0.13 . Thus, the data readily reveals that conditioner treatment decreases coefficient for friction of hair. The main mechanism for this macroscale trend is that lubrication with a thin conditioner layer occurs over a large contact area, and thus the conditioner layer shears easily to create a lubricious effect [LaTorre and Bhushan, 2005b]. It is important to note that the magnitude of the coefficient of friction values is higher on the macroscale than on the other scales for all hair types. Bhushan et al. [2004] have previously outlined several differences in operating conditions which can responsible for higher macroscale friction values. The one most relevant to our situation is that coefficient of friction increases with an increase in the AFM tip radius. Nanoscale friction data is taken with a sharp AFM tip, while the macro- and microscale tests have contacts which range from nanoasperities to much larger asperities which may be responsible for larger values of friction force on these scales. The combination of higher normal loads with a larger contact area (due to contact with multiple fibers at the same time) may also be responsible for increased coefficient of friction on the macroscale. 
Summary of coefficient of friction and adhesive force data on various scales

Coefficient of friction
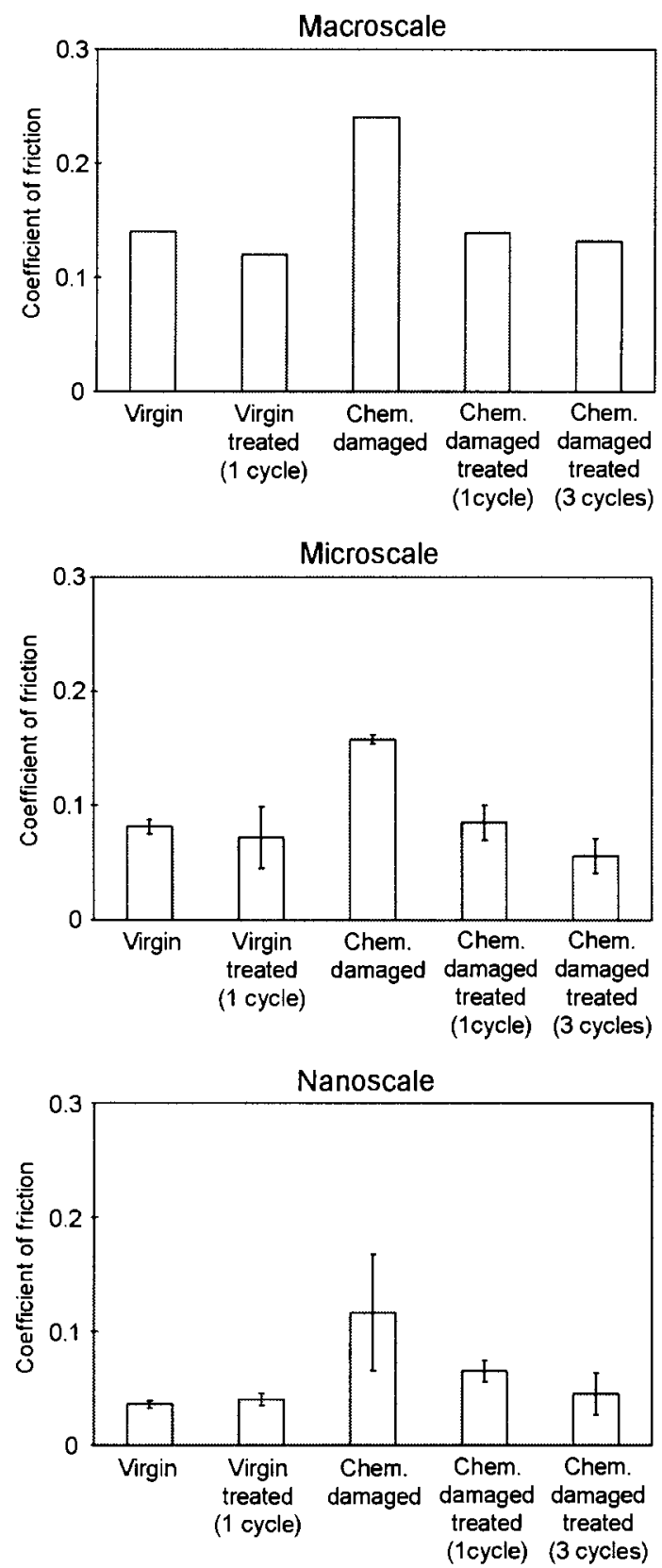

Adhesive force
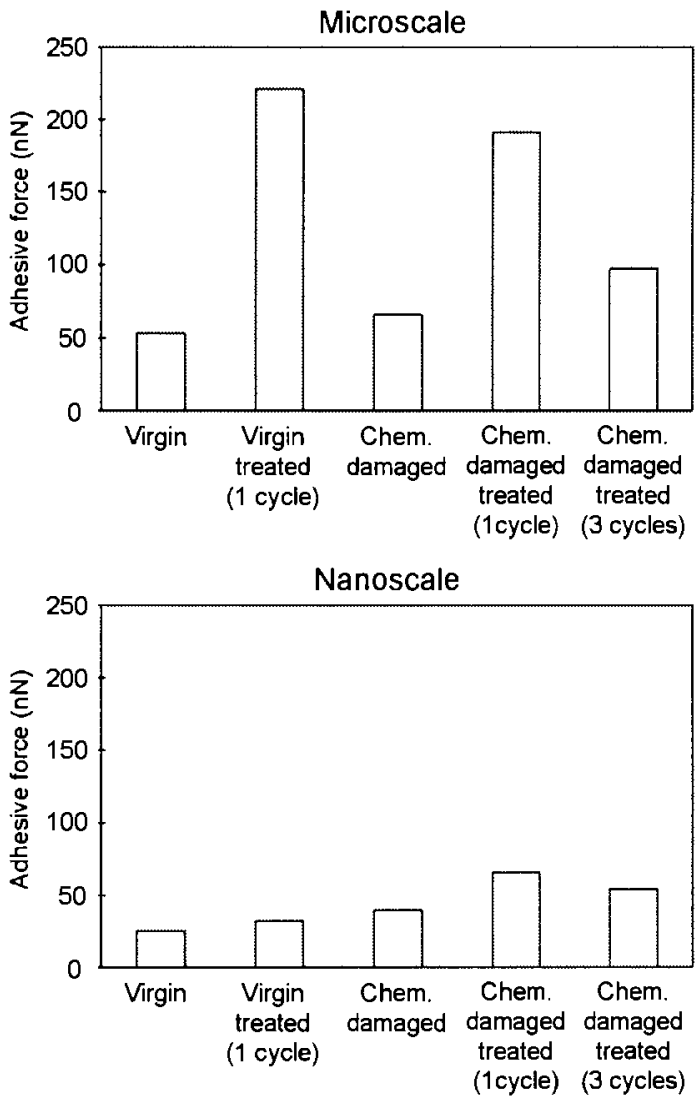

Fig. 4.22 Summary of coefficient of friction and adhesive force data on various scales. 


\begin{tabular}{|l|c|c|c|c|c|c|}
\hline & \multicolumn{3}{|c|}{ Coefficient of friction } & \multicolumn{3}{c|}{ Adhesive force (nN) } \\
\hline Hair sample & Macroscale & Microscale & Nanoscale & Macroscale & Microscale & Nanoscale \\
\hline $\begin{array}{l}\text { Virgin } \\
\text { (untreated) }\end{array}$ & 0.14 & $\begin{array}{c}0.08 \\
\pm 0.01\end{array}$ & $\begin{array}{c}0.04 \\
\pm 0.003\end{array}$ & - & 53 & 25 \\
\hline $\begin{array}{l}\text { Virgin treated } \\
\text { (1 cycle } \\
\text { commercial } \\
\text { conditioner) }\end{array}$ & 0.12 & $\begin{array}{c}0.07 \\
\pm 0.03\end{array}$ & $\begin{array}{c}0.04 \\
\pm 0.01\end{array}$ & - & 221 & 32 \\
\hline $\begin{array}{l}\text { Chemically } \\
\text { damaged } \\
\text { (untreated) }\end{array}$ & 0.24 & $\begin{array}{c}0.16 \\
\pm 0.003\end{array}$ & $\begin{array}{c}0.12 \\
\pm 0.05\end{array}$ & - & 66 & 40 \\
\hline $\begin{array}{l}\text { Chemically } \\
\text { damaged treated } \\
(1 \text { cycle } \\
\text { commercial } \\
\text { conditioner) }\end{array}$ & 0.14 & $\begin{array}{c}0.08 \\
\pm 0.02\end{array}$ & $\begin{array}{c}0.07 \\
\pm 0.01\end{array}$ & - & 191 & 66 \\
\hline $\begin{array}{l}\text { Chemically } \\
\text { damaged treated } \\
\text { (3 cycle } \\
\text { commercial } \\
\text { conditioner) }\end{array}$ & 0.13 & $\begin{array}{c}0.06 \\
\pm 0.02\end{array}$ & $\begin{array}{c}0.04 \\
\pm 0.02\end{array}$ & - & 97 & 54 \\
\hline
\end{tabular}

Table 4.5 Coefficient of friction and adhesive force of hair on various scales

The coefficient of friction trends are similar on the microscale. On the microscale, virgin hair coefficient of friction was 0.08 , while application of 1 cycle conditioner decreased the coefficient of friction only slightly to 0.07 . From Figure 4.22 it is important to note the large standard deviation on the virgin treated value. The corresponding adhesive force data in the same row is useful to better understand this behavior. It is shown that the virgin treated hair has a large adhesive force contribution (due to meniscus effects caused by the conditioner layer) which is having significant effects on the variation of friction force, and consequently the coefficient of friction. The 
chemically damaged hair has the largest coefficient of friction of the set, 0.16 .

Application of 1 conditioner cycle brought the value down to 0.08 , while it was even lower for 3 conditioner cycles, 0.06 . Adhesive force increased significantly with conditioner application; for virgin hair, adhesive force increased from about $50 \mathrm{nN}$ to 220 $\mathrm{nN}$ due to the meniscus effects that come about from AFM tip interaction with the conditioner on the cuticle surface. Likewise, the adhesive force for chemically damaged hair was about $65 \mathrm{nN}$ and jumped to $190 \mathrm{nN}$ and $100 \mathrm{nN}$ for 1 and 3 cycles of conditioner treatment, respectively. A possible reason that one cycle of conditioner on damaged hair showed higher average adhesive force than three cycles could be because the three cycles generally places the conditioner more uniformly on the surface rather than accumulating it most on the bottom surface near the cuticle edge, which is where the adhesive force maps were generally taken. Nevertheless, the increased adhesive force shown in the plots is a clear indication of conditioner present on the hair surface [LaTorre and Bhushan, 2005b].

On the nanoscale, coefficient of friction of virgin and virgin treated hair is similar, but slightly higher for the treated cases. This is opposite of the trend on the macroscale and slightly different than that observed on the microscale. These meniscus bridges require more force to break through than with untreated hair, which causes the coefficient of friction to be similar to the untreated value, instead of experiencing a significant decrease which is traditionally expected. Damaged hair shows a much higher coefficient of friction, and with more variation in the values since the chemical damage varies throughout each individual fiber. Contrary to the virgin and virgin treated hair nanoscale 
results, coefficient of friction for damaged hair decreased with application of conditioner treatment (both 1 and 3 cycles), which agrees well with macro- and microscale trends.

There are several reasons that nanoscale coefficient of friction trends for virgin treated and chemically damaged treated hair are different. In order to serve as a reference for this discussion, Figure 4.9 and Figure 4.10 show surface roughness, friction force, and adhesive force maps for the various hair (taken with a nanoscale AFM tip) [LaTorre and Bhushan, 2005b]. The effects of chemical damage play a large role. It is widely known that the cuticle surface of any hair is negatively charged. This charge becomes even more negative with the application of chemical damage to the hair. As a result, the positively charged particles of conditioner have even stronger attraction to the chemically damaged surface, which explains the increased presence of conditioner when compared to virgin treated hair. With the application of three conditioner cycles on damaged treated hair, there is an even more uniform placement of the conditioner [LaTorre and Bhushan, 2005b; LaTorre et al., 2006]. This leads to high adhesive force due to meniscus effects (similar to that of virgin treated hair) but more importantly, lower shear strength on the surface. This creates an overall effect of lubrication as the tip travels across the cuticle surface and ultimately lowers the coefficient of friction.

Another reason for the difference in nanoscale trends between virgin and chemically damaged hair may have to do with drastic differences in hydrophobicity of the two hair types. Virgin hair has been shown to be hydrophobic, with a contact angle around $100^{\circ}$ (see Table 3.4). Chemically damaged hair, however, is hydrophilic, with a contact angle around $60^{\circ}$. The conditioner gel network is primarily composed of water, together with fatty alcohols, cationic surfactants, and silicones. Thus, the hydrophobicity 
of the hair will be relevant to not only how much conditioner is deposited, but also how it diffuses into the hair and bonds to the hair surface. For virgin treated hair, the conditioner deposits in certain locations, especially near the cuticle edge, but due to the hydrophobicity of the cuticle does not spread out as readily as with chemically damaged hair. For chemically damaged hair, the conditioner spreads out a bit more uniformly and in more places over the cuticle surface due to both the hydrophilicity and the stronger negative charge which attracts more conditioner deposition. Thus, as the tip scans over the virgin treated surface, the conditioner does not smear as readily, causing the tip to have to break the tiny meniscus bridges formed with the conditioner. This results in increased adhesive force, which contributes to higher friction force. This ends ups increasing the coefficient of friction to about the same level as the untreated virgin hair, instead of a reduction in coefficient which is typically expected for a lubricated surface. In the case of chemically damaged hair, however, the conditioner layer is already more spread out, especially in the case of 3 cycles of treatment, as shown in the friction force maps of Figure 4.9. As the tip scans over the surface, the overall effect is one of reduced shear strength, i.e. the conditioner layer, albeit not fully continuous, smears with tip travel and causes reduced coefficient of friction between the tip and the hair. With 3 cycles the conditioner thickness increases slightly [Chen and Bhushan, 2005b], and the layer is even more uniformly distributed over the surface [LaTorre and Bhushan, 2005b], which causes a further reduction in coefficient of friction, much like the results seen on both the microand macroscale.

Figure 4.23 shows schematically the mechanisms responsible for the reverse trends seen on the nanoscale for virgin treated hair, and Table 4.6 summarizes the 
observations seen for both directionality dependence and scale effects. In the top cartoon of Figure 4.23, the thin layer of conditioner acts as a lubricant over the hair fiber, limiting the dry contact with the synthetic skin block and creating easier relative motion, which decreases coefficient of friction compared to the untreated hair. This is true on the macroscale for both virgin and chemically damaged hair. On the microscale, the same trend is experienced; that is, the $4 \mu \mathrm{m}$ radius of the AFM ball comes in contact with multiple cuticle scales at the same time, causing an overall lubrication effect for both virgin treated and chemically damaged treated hair as the thin conditioner layer is sheared to created easier relative motion. It is important to note, however, that adhesive forces due to meniscus effects are the same magnitude as the applied microscale normal load.

On the nanoscale (bottom cartoon of Figure 4.23), we see different trends for virgin treated and chemically damaged treated hair. As discussed earlier, the hydrophobicity of the virgin hair causes different deposition of conditioner. The AFM tip has to break the tiny meniscus bridges formed with the conditioner as it scans across the hair surface, which increases adhesive force contribution and results in an increased coefficient of friction. For hydrophilic chemically damaged hair, there is more uniform deposition and better smearing of the conditioner layer, which serves to lower coefficient of friction between the tip and the cuticle surface. 
Mechanisms for various coefficient of friction trends for macro-, micro-, and nanoscales

\section{Macroscale}

Conditioner application decreases coefficient of friction

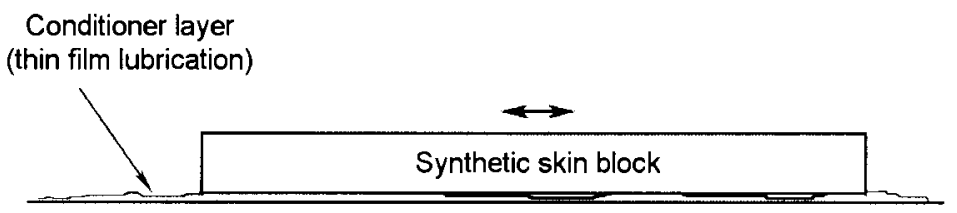

Treated hair fiber (virgin or chemically damaged)

\section{Microscale}

Conditioner application decreases coefficient of friction

Conditioner layer (thin film lubrication)

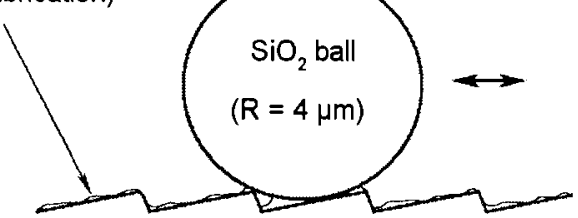

Treated hair fiber (virgin or chemically damaged)

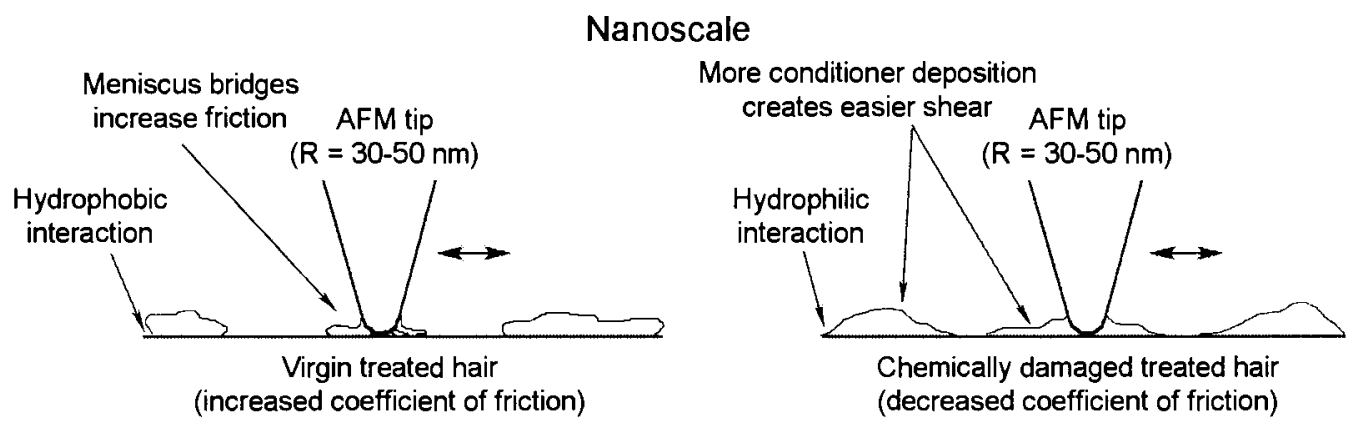

Fig. 4.23 Schematic of various mechanisms responsible for scale dependence of hair friction and adhesion. 


\begin{tabular}{|c|c|}
\hline Observation & Mechanism \\
\hline \multicolumn{2}{|c|}{ Directionality dependence of coefficient of friction } \\
\hline \multirow{3}{*}{$\begin{array}{l}\text { The coefficient of friction is always } \\
\text { higher in the "against cuticle" direction } \\
\text { as compared to the "along cuticle" } \\
\text { direction. }\end{array}$} & $\begin{array}{l}\text { Macroscale: Thousands of scale edges resist motion (creating } \\
\text { higher friction forces) as they are forced backwards and uplifted. }\end{array}$ \\
\hline & $\begin{array}{l}\text { Microscale: Scale edges come in contact with the AFM } \\
\text { microscale tip and causing local collisions and ratcheting of the } \\
\text { tip, which create higher friction. }\end{array}$ \\
\hline & $\begin{array}{l}\text { Nanoscale: AFM nanoscale tip collides with the scale edge and } \\
\text { has to climb up the edge, resulting in a high friction signal. }\end{array}$ \\
\hline \multicolumn{2}{|r|}{ Scale effects } \\
\hline $\begin{array}{l}\text { Coefficient of friction values are largest } \\
\text { on the macroscale, followed by } \\
\text { microscale and then nanoscale values. }\end{array}$ & $\begin{array}{l}\text { Coefficient of friction increases with an increase in the AFM tip } \\
\text { radius. Nanoscale friction data is taken with a sharp AFM tip, } \\
\text { while the macro- and microscale tests have contacts which range } \\
\text { from nanoasperities to much larger asperities. Macroscale has } \\
\text { contact with multiple fibers at the same time. }\end{array}$ \\
\hline $\begin{array}{l}\text { Adhesive force values are larger on the } \\
\text { microscale than on the nanoscale. }\end{array}$ & $\begin{array}{l}\text { The increase in adhesive force on the microscale is due to the } \\
\text { increased radius of the microscale ball, which consequently } \\
\text { induces larger meniscus force contribution to the adhesive force. }\end{array}$ \\
\hline \multirow{3}{*}{$\begin{array}{l}\text { On all scales, coefficient of friction } \\
\text { decreases for chemically damaged } \\
\text { treated hair compared to untreated. The } \\
\text { same trend occurs for virgin treated hair } \\
\text { on the macro- and microscales, but on } \\
\text { nanoscale value is same as untreated. }\end{array}$} & $\begin{array}{l}\text { Macroscale: Thin layer of conditioner acts as a lubricant over } \\
\text { the hair fiber (decreases coefficient of friction) (whether virgin } \\
\text { or chemically damaged). }\end{array}$ \\
\hline & $\begin{array}{l}\text { Microscale: Same trend at macroscale is experienced because } \\
\text { AFM microscale tip comes in contact with multiple cuticle } \\
\text { scales at the same time, causing an overall lubrication effect. }\end{array}$ \\
\hline & $\begin{array}{l}\text { Nanoscale: Hydrophobicity of the virgin hair causes different } \\
\text { deposition of conditioner. AFM tip has to break the tiny } \\
\text { meniscus bridges when scanning increasing adhesive force } \\
\text { coefficient of friction. For chemically damaged hair, higher } \\
\text { negative charge and a hydrophilic surface results in more } \\
\text { uniform deposition and better smearing of the conditioner layer } \\
\text { lower coefficient of friction. }\end{array}$ \\
\hline $\begin{array}{l}\text { On both micro- and nanoscales, the } \\
\text { adhesive force increases for treated } \\
\text { hair. }\end{array}$ & $\begin{array}{l}\text { Meniscus forces brought about by the presence of the } \\
\text { conditioner layer increase adhesive force. }\end{array}$ \\
\hline
\end{tabular}

Table 4.6 Observations and corresponding mechanisms regarding coefficient of friction and adhesion for various hair treatments. 


\subsection{Surface roughness and friction of skin}

Synthetic materials were also studied for surface roughness and friction force information, shown in Figure 4.24(a). While macroscale dimples could be seen on the surface of collagen film, it was interesting to find similar pits and dimples on the micro/nanoscale, consequently with a large variation in dimple size and depth. Polyurethane films are shown to have quite different topography and friction forces, while their coefficient of friction is very similar. Human skin shows a rougher texture with higher peaks, Figure 4.24(b). The roughness parameters for the collagen and polyurethane films, and also for human skin, are presented in Table 4.7 [LaTorre and Bhushan, 2005a]. Vertical height standard deviation $\sigma$ was approximately 3 times larger than that of virgin hair for both synthetic materials. However, the correlation length $\beta^{*}$ was lower than what was typically observed in hair. The average coefficient of friction for these synthetic materials are shown in Figure 4.25 plotted next to virgin Caucasian hair as a reference. These values were calculated using the slope of the friction force curves, described previously. Both collagen and polyurethane films displayed similar coefficient of friction values of 0.22 and 0.24 , respectively. Virgin hair displays a much lower coefficient of friction than both materials, approximately eight times lower. 


\section{Surface roughness and friction force maps of collagen and polyurethane films}

Collagen film (synthetic hair)
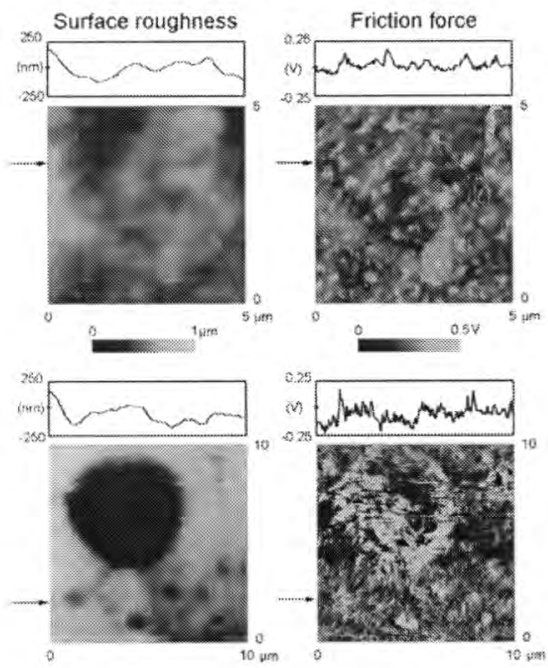

Polyurethane film (synthetic skin)
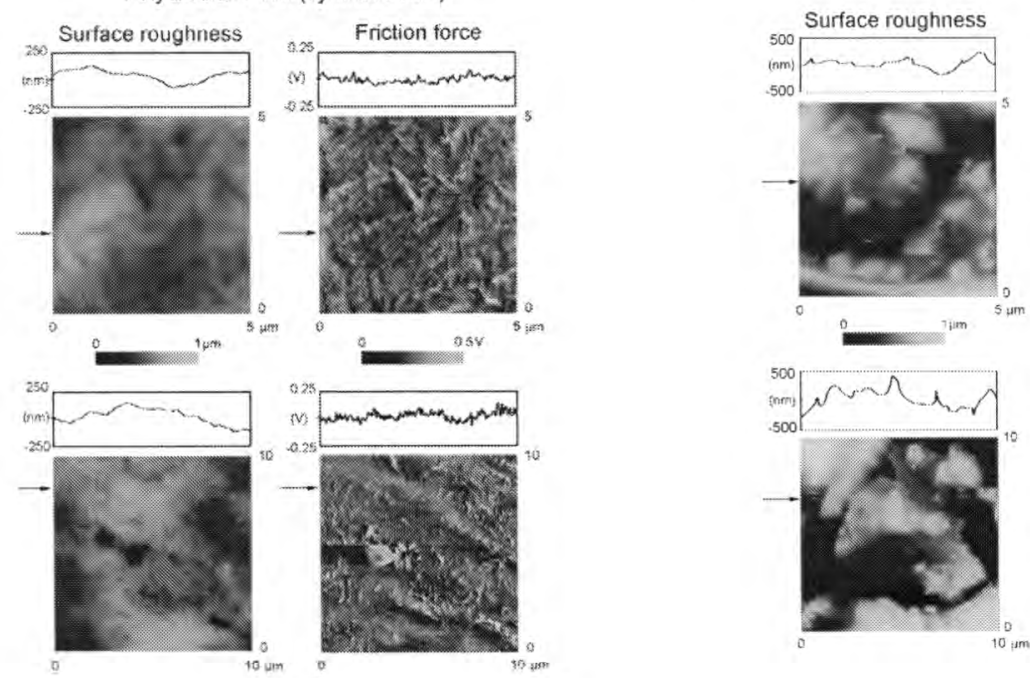

(a)

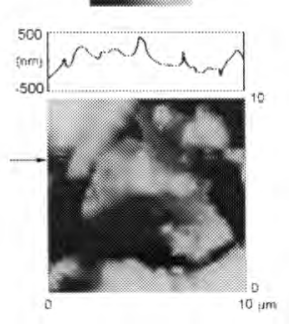

(b)

Fig. 4.24 (a) Surface roughness and friction force images for collagen and polyurethane films at 5 and $10 \mu \mathrm{m}^{2}$ scan sizes (b) Surface roughness for human skin at 5 and $10 \mu \mathrm{m}^{2}$ scan sizes. Note that vertical scales in 2D section profiles are doubled [LaTorre and Bhushan, 2005a]. 
Coefficient of friction comparison for collagen and polyurethane films

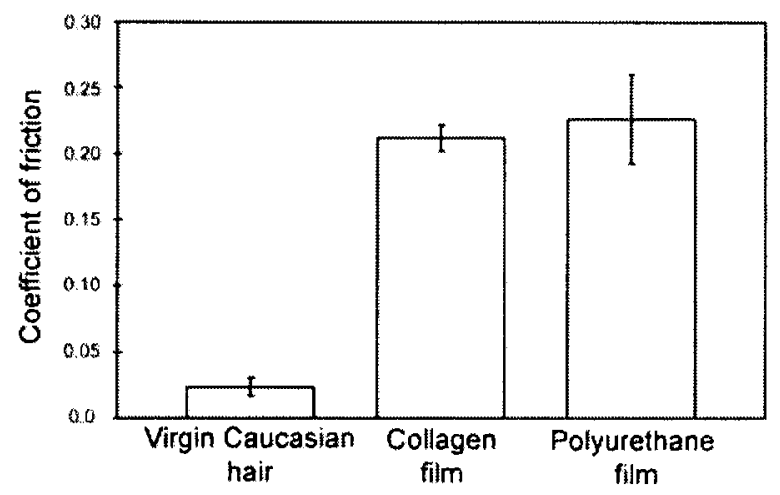

Fig. 4.25 Average coefficient of friction values for collagen and polyurethane films.

Error bars represent $\pm 1 \sigma$ on the average coefficient value [LaTorre and Bhushan, 2005a]. 


\begin{tabular}{|c|c|c|}
\hline \multicolumn{3}{|c|}{$\begin{array}{c}\text { Surface roughness parameters } \sigma, \beta^{*} \text { for } \\
\text { collagen and polyurethane films and human skin }\end{array}$} \\
\hline & $\sigma(\mathrm{nm})$ & $\beta^{*}(\mu \mathrm{m})$ \\
\hline $\begin{array}{c}\text { Collagen } \\
\text { (synthetic hair) }\end{array}$ & $36 \pm 11$ & $0.50 \pm 0.1$ \\
\hline $\begin{array}{c}\text { Polyurethane } \\
\text { (synthetic skin) }\end{array}$ & $33 \pm 6$ & $0.71 \pm 0.1$ \\
\hline Human skin & $80 \pm 28$ & $0.59 \pm 0.2$ \\
\hline
\end{tabular}

Table 4.7 Surface roughness parameters $\sigma, \beta^{*}$ for collagen and polyurethane films and human skin. 


\section{CHAPTER 5}

\section{CONCLUSIONS}

AFM contact mode has been used to perform nanotribological studies on various hair and skin. Friction force and the resulting coefficient of friction are seen to be higher on chemo-mechanically damaged hair than on virgin hair, due to the increase in surface roughness and a change in surface properties that results from exposure to chemical damage. Generally speaking, the average coefficient of friction is similar between virgin and virgin treated hair of each ethnicity. However, in virgin treated hair there is an increase in friction forces around the cuticle edges and surrounding area. It is currently believed that the increase in friction force is due in part to an increase in meniscus effects which increase the adhesive force contribution to friction at sites where conditioner is deposited or accumulated on the surface, namely around the cuticle scale edges. This adhesive force is of the same magnitude as the normal load, which makes the adhesive force contribution to friction rather significant. On the macroscale, however, the adhesive force is much lower in magnitude than the applied normal load, so the adhesive force contribution to friction is negligible over the hair switch. As a result, treated hair shows a decrease in friction force on the macroscale, which is opposite the micro/nanoscale trend. The friction and adhesion data on the micro/nanoscale is useful, though, because it relates to the presence of conditioner on the cuticle surface and allows 
for obtaining an estimate of conditioner distribution. Studies using force calibration plot technique showed a decrease in adhesive force with damaged hair, and significantly higher adhesive force for treated hair. This increase on the micro/nanoscale is most likely due to meniscus force contributions from the accumulation and localization of a conditioner layer on the hair surface. Thus, the presence of conditioner can be detected by this increasing adhesive force. The directionality dependence of friction is evident when the cuticle edge is examined using FFM.

Chemically damaged treated hair shows a much stronger affinity to conditioner than virgin hair. The negative charge of hair fibers becomes even more negative with the application of chemical damage to the hair. As a result, the positively charged particles of conditioner have even stronger attraction to the chemically damaged surface, and this results in an increased presence of conditioner (and corresponding higher friction forces) when compared to virgin treated hair. With the application of three conditioner cycles on chemically damaged treated hair, friction force increases all over the cuticle surface, showing a more uniform placement of the conditioner. Contrary to the virgin and virgin treated hair results, coefficient of friction for chemically damaged hair decreased with application of commercial conditioner treatment (both one and three cycles). One possible explanation is that because the stronger negative charge on damaged hair results in better attraction of conditioner, this leads to higher adhesive force but, more importantly, lower shear strength on the surface.

Environmental effects were studied for various hair. The coefficient of friction generally decreased with increasing temperature. After soaking hair in de-ionized water, virgin hair exhibits a decrease in coefficient of friction after soaking. Virgin hair is more 
hydrophobic (based on contact angle data), so more of the water is present on the surface and results in a lubrication effect after soaking. Chemically damaged hair tends to be hydrophilic due to the chemical degradation of the cuticle surface, and results in absorption of water after soaking. This softens the hair, which leads to higher friction, even with conditioner treatment. Durability tests show that once conditioner is applied to virgin hair, wear does not show up as an increase in friction force. Thus, conditioner serves as a protective covering to the virgin hair and helps protect the tribological properties when wear ensues.

In most cases, a decrease in coefficient of friction was observed on chemically damaged hair with the addition of the PDMS blend or amino silicones to the BTMAC surfactant. The silicones are typically used as a major source of lubrication and thus give the conditioner more mobility on the hair surface compared to just surfactants and fatty alcohols. The inverse trend was seen only for the amino silicone group at high deposition. The dampened mobility of the amino silicone at high deposition levels, with respect to hair surface and tip, may account for this wide variation in coefficient of friction. Adhesive force varied widely, but typically showed a significant increase with the presence of conditioner ingredients. This is a clear sign that meniscus effects are influencing the pull-off force between the tip and the sample. At high deposition levels, the amino silicones showed much more distinct regions of high and low friction and adhesion, which shows that there is less mobility of these molecules and much less redistribution as they coat the hair.

Investigations of scale effects and directionality dependence on friction and adhesion have been studied. Macroscale friction force data clearly shows directionality 
effects. Thousands of scale edges come in contact with the synthetic skin. When traveling against cuticle, edges resist motion (creating higher friction forces) as they are forced backwards and uplifted from their interface with the underlying cuticle layers.

On the microscale, when scanning against the cuticle, distinctly large spikes in the data are observed at roughly $5-10 \mu \mathrm{m}$ intervals. This is clearly the effects of the scale edges coming in contact with the AFM microscale tip and causing local collisions and ratcheting of the tip, which create higher friction. On the nanoscale, AFM nanoscale tip collides with the scale edge and has to climb up the edge, resulting in a high friction signal.

Coefficient of friction values are largest on the macroscale, followed by microscale and then nanoscale values. In general, coefficient of friction increases with an increase in the AFM tip radius. Nanoscale friction data is taken with a sharp AFM tip, while the macro- and microscale tests have contacts which range from nanoasperities to much larger asperities which may be responsible for larger values of friction force on these scales. Adhesive force values are larger on the microscale than on the nanoscale. The increase in adhesive force calculated by force calibration plots with the microscale AFM tip compared to the nanoscale AFM tip is in large part due to the increased radius of the microscale ball, which consequently induces larger meniscus force contribution to the adhesive force.

On all scales, coefficient of friction decreases for chemically damaged treated hair compared to untreated. The same trend occurs for virgin treated hair on the macro- and microscales, but not on the nanoscale. On the macroscale, the thin layer of conditioner acts as a lubricant over the hair fiber, which decreases coefficient of friction compared to 
the untreated hair (whether virgin or chemically damaged). On the microscale, the same trend is experienced because AFM microscale tip comes in contact with multiple cuticle scales at the same time, causing an overall lubrication effect. On the nanoscale, the hydrophobicity of the virgin hair causes different deposition of conditioner. The AFM tip has to break the tiny meniscus bridges formed with the conditioner as it scans across the hair surface, which increases adhesive force contribution and results in an increased coefficient of friction. For chemically damaged hair, there is higher negative charge and a hydrophilic surface, which results in more uniform deposition and better smearing of the conditioner layer, which serves to lower coefficient of friction between the tip and the cuticle surface.

On both micro- and nanoscales, the adhesive force increases with application of conditioner (for both virgin and chemically damaged hair). This is clearly the effects of meniscus forces brought about by the presence of the conditioner layer on the cuticle surface which interacts with the tip. 


\section{LIST OF REFERENCES}

H. A. Barnes and G. P. Roberts, "The non-linear viscoelastic behaviour of human hair at moderate extensions", Inter. J. Cosmet. Sci. 22 (2000) 259-264

B. Bhushan: Principles and Applications of Tribology (Wiley, New York 1999a)

B. Bhushan: Handbook of Micro/Nanotribology, $2^{\text {nd }}$ Ed. (CRC Press, Boca Raton, FL 1999b)

B. Bhushan: Introduction to Tribology (Wiley, New York 2002)

B. Bhushan, (ed.): Springer Handbook of Nanotechnology (Springer-Verlag, Heidelberg, Germany 2004)

B. Bhushan: Nanotribology and Nanomechanics-An Introduction (Springer-Verlag, Heidelberg, Germany 2005)

B. Bhushan and Z. Burton, "Adhesion and friction properties of polymers in microfluidic devices”, Nanotechnology 16 (2005) 467-478

B. Bhushan and C. Dandavate, "Thin-film friction and adhesion studies using atomic force microscopy," J. Appl. Phys. 87 (2000) 1201-1210

B. Bhushan, H. Liu, S. M. Hsu, "Adhesion and Friction Studies of Silicon and Hydrophobic and Low Friction Films and Investigation of Scale Effects", ASME J. Tribol. 126 (2004) 583-590

B. Bhushan, G. Wei, and P. Haddad, "Friction and wear studies of human hair and skin," Wear (2005) in press

C. Bolduc and J. Shapiro, "Hair Care Products: Waving, Straightening, Conditioning, and Coloring”, Clinics in Dermatology 19 (2001) 431-436.

N. H. Chen and B. Bhushan, "Cellular structural characterization of human hair and conditioner distribution using torsional resonance mode with an AFM", submitted for publication (2005a) 
N. H. Chen and B. Bhushan, "AFM studies of conditioner thickness distribution and binding interaction on hair surface", submitted for publication (2005b)

A. Feughelman: Mechanical Properties and Structure of Alpha-Keratin Fibres: Wool, Human Hair and Related Fibres (University of South Wales Press, Sydney, 1997)

M. E. Ginn, C. M. Noyes and E. Jungermann, "The Contact Angle of Water on Viable Human Skin", Journal of Colloid and Interface Science 26 (1968)146-151

J. Gray, "Hair Care and Hair Care Products", Clinics in Dermatology 19 (2001) 227-236

J. Gray: The World of Hair (Online, http://www.pg.com/science/haircare/hair_twh_toc.htm, 2003)

J. Jachowicz and R. McMullen, "Mechanical analysis of elasticity and flexibility of virgin and polymer-treated hair fiber assemblies", J. Cosmet. Sci. 53 (2002) 345-361

C. Jalbert, J. T. Koberstein, I.Yilgor, P. Gallagher, and V. Krukonis, "Molecular Weight Dependence and End-Group Effects on the Surface Tension of Poly(dimethylsiloxane)", Macromolecules 26 (1993) 3069-3074

P. Jollès, H. Zahn and H. Höcker (Eds.): Formation and Structure of Human Hair (Birkhäuser Verlag, Berlin 1997)

C. LaTorre and B. Bhushan, "Nanotribological characterization of human hair and skin using atomic force microscopy", Ultramicroscopy (2005a) in press

C. LaTorre and B. Bhushan, "Nanotribological effects of hair care products and environment on human hair using atomic force microscopy", J. Vac. Sci. Technol., A (2005b) in press

C. LaTorre and B. Bhushan, "Investigation of scale effects and directionality dependence on friction and adhesion of human hair using AFM and macroscale friction test apparatus", submitted for publication (2006)

C. LaTorre, B. Bhushan, P.M. Torgerson, J. Yang, "Nanotribological effects of silicone type and deposition level and surfactant type on human hair using atomic force microscopy", submitted for publication (2006)

G. Lerebour, S. Cupferman, C. Cohen, and M.N. Bellon-Fontaine, "Comparison of surface free energy between reconstructed human epidermis and in situ human skin", Skin Research and Technology 6 (2000) 245-249 
H. Liu and B. Bhushan, "Nanotribological characterization of molecularly thick lubricant films for applications to MEMS/NEMS by AFM," Ultramicroscopy 97 (2003) 321-340

R. Molina, F. Comelles, M. R. Julia, and P. Erra, "Chemical Modifications on Human Hair Studied by Means of Contact Angle Determination", Journal of Colloid and Interface Science 237 (2001) 40-46

P. T. Pugliese: Physiology of the Skin (Allured Publishing Corporation, Carol Stream, Illinois 1996)

R. J. Randebrook, J. Soc. Cosmet. Chem. 15 (1964) 691

C. Robbins: Chemical and Physical Behavior of Human Hair ( $3^{\text {rd }}$ Edition, SpringerVerlag, New York 1994)

H. Schott, "Contact Angles and Wettability of Human Skin", Journal of Pharmaceutical Sciences 60 (1971) 1893-1895

J. R. Smith and J. A. Swift, "Lamellar subcomponents of the cuticular cell membrane complex of mammalian keratin fibres show friction and hardness contrast by AFM", J. Microscopy 206 (2002) 182-193

J. A. Swift, "Fine Details on the Surface of Human Hair," Int. J. Cosmetic Sci. 13 (1991) 143-159

J. A. Swift: Morphology and Histochemistry of Human Hair, Formation and Structure of Human Hair, ed. By P. Jolles, H. Zahn, and H. Hocker (Birkhauser Verlag, Berlin 1997) $149-175$

J. A. Swift, "The mechanics of fracture of human hair", Inter. J. Cosmet. Sci. 21 (1999) 227- 239

J. A. Swift, "The cuticle controls bending stiffness of hair", J. Cosmet. Sci. 51 (2000) $37-$ 38

A. N. Syed, A. Kuhajda, H. Ayoub, K. Ahmad, and E. M. Frank, "African-American Hair: Its physical properties and differences relative to Caucasian hair", in Hair Care (Cosmetics \& Toiletries Applied Research Series) (Allured Publishing Corporation, Carol Stream, IL 1996)

G. Wei, B. Bhushan and P. M. Torgerson, "Nanomechanical characterization of human hair using nanoindentation and SEM", Ultramicroscopy (2005) in press 
P. W. Wertz, D. T. Downing, "Stratum corneum: biological and biochemical considerations", Transdermal Drug Delivery, J. Swarbrick, R. H. Guy (eds). (Marcel Dekker, New York, 1989)

P. W. Wertz, K. C. Madison, D. T. Downing, "Covalently Bound Lipids of Human Stratum Corneum”, J Invest Dermatol 92 (1989) 109

H. Yanazawa, "Adhesion model and experimental-verification for polymer SIO2 system", Colloids and Surfaces 9 (1984) 133-145

C. Zviak (ed.): The Science of Hair Care (Marcel Dekker, New York 1986) 


\section{APPENDIX A \\ SHAMPOO AND CONDITIONER TREATMENT PROCEDURE}


This appendix section outlines the steps involved in washing hair switches with shampoo and/or conditioner.

- Shampoo treatments

Shampoo treatments consisted of applying a commercial shampoo evenly down a hair switch with a syringe. Hair was lathered for 30 seconds, rinsed with tap water for 30 seconds, then repeated. The amount of shampoo used for each hair switch was $0.1 \mathrm{~cm}^{3}$ shampoo per gram of hair. Switches were hanged to dry in an environmentally controlled laboratory, and then wrapped in aluminum foil.

\section{- Conditioner treatments}

A commercial conditioner was applied $0.1 \mathrm{~cm}^{3}$ of conditioner per gram of hair. The conditioner was applied in a downward direction (scalp to tip) thoroughly throughout hair switch for 30 seconds, and then allowed to sit on hair for another 30 seconds. The switch was then rinsed thoroughly for 30 seconds. Switches were hanged to dry in an environmentally controlled laboratory, and then wrapped in aluminum foil. 
APPENDIX B

CONDITIONER THICKNESS APPROXIMATION 
A cylindrical hair fiber of diameter $D=50 \mu \mathrm{m}$ (radius $R=25 \mu \mathrm{m}$ ) is considered. For conditioner thickness calculations, the following assumptions are made: (1) hair and the material being added have the same density, (2) coating of material is uniform on the hair surface, (3) the cross-sectional area of a hair fiber remains constant along the longitudinal axis of the fiber (i.e. from root to tip); the hair fiber is perfectly cylindrical (circular cross-section), and (4) the deposited conditioner remains bonded to the cuticle surface (no absorption into the cuticle layer).

The cross-sectional area of an untreated hair fiber is initially calculated. By adding a specified amount of conditioner, this area will increase and cause the radius of the hair treated fiber to increase. This increase in the radius of the treated hair will be equivalent to the thickness of the conditioner layer. The original cross-sectional area $A_{c}$ of hair fiber is

$$
A_{c}=\pi R^{2}=\pi(25 \mu m)^{2}=1963.4954 \mu m^{2}
$$

Adding $200 \mathrm{ppm}$ material to the surface (which is comparable to the amount that commercial conditioners typically deposit) will cause an increase in volume (for a unit fiber length) by $200 \mathrm{ppm}$, or by 0.0002 . Thus, the cross - sectional area $A_{c, \text { conditioner }}$ of the treated hair will increase by the same amount to

$$
A_{c, \text { conditioner }}=1.0002 A_{c}=1963.888 \mu \mathrm{m}^{2}
$$

which results in a new radius $R_{\text {conditioner, }}$

$$
R_{\text {conditioner }}=\sqrt{\frac{A_{c, \text { conditioner }}}{\pi}}=25.0025 \mu \mathrm{m}
$$


Therefore, subtracting the original radius from the radius after treatment increases the thickness of the hair by 0.0025 microns, or $2.5 \mathrm{~nm}$.

It is important to note that the approximation of the conditioner thickness as 2.5 $\mathrm{nm}$ was determined for a particular hair diameter and material deposition amount (with the hair and material having equal densities). Although these are generally realistic approximations, hair diameter often varies by a factor of 2 and the deposition level can vary up to an order of magnitude. The conditioner layer has been shown in previous work to be nonuniform as well. Thus, actual conditioner thickness can deviate significantly from this number. 